\title{
A Herschel Space Observatory Spectral Line Survey of Local Luminous Infrared Galaxies from 194 to 671 Microns*
}

\author{
Nanyao $\mathrm{Lu}^{1,2,3}$, Yinghe Zhao ${ }^{4,5,6,3}$, Tanio Díaz-Santos ${ }^{7}$, C. Kevin $\mathrm{Xu}^{1,2,3}, \mathrm{Yu} \mathrm{Gao}^{6}$, Lee Armus ${ }^{8}$, Kate G. Isaak ${ }^{9}$, \\ Joseph M. Mazzarella ${ }^{3}$, Paul P. van der Werf ${ }^{10}$, Philip N. Appleton ${ }^{3}$, Vassilis Charmandaris ${ }^{11,12}$, Aaron S. Evans ${ }^{13,14}$, \\ Justin Howell ${ }^{3}$, Kazushi Iwasawa ${ }^{15,16}$, Jamie Leech ${ }^{17}$, Steven Lord ${ }^{18}$, Andreea O. Petric ${ }^{19}$, George C. Privon ${ }^{20,21}$, \\ David B. Sanders ${ }^{22}$, Bernhard Schulz ${ }^{3}$, and Jason A. Surace ${ }^{8}$ \\ ${ }^{1}$ National Astronomical Observatories, Chinese Academy of Sciences (CAS), Beijing 100012, China; nanyao.lu@gmail.com \\ ${ }^{2}$ South American Center for Astronomy, CAS, Camino El Observatorio 1515, Las Condes, Santiago, Chile \\ ${ }^{3}$ Infrared Processing and Analysis Center, California Institute of Technology, MS 100-22, Pasadena, CA 91125, USA \\ ${ }^{4}$ Yunnan Observatories, CAS, Kunming 650011, China \\ ${ }^{5}$ Key Laboratory for the Structure and Evolution of Celestial Objects, CAS, Kunming 650011, China \\ ${ }^{6}$ Purple Mountain Observatory, CAS, Nanjing 210008, China \\ ${ }^{7}$ Nucleo de Astronomia de la Facultad de Ingenieria, Universidad Diego Portales, Av. Ejercito Libertador 441, Santiago, Chile \\ ${ }^{8}$ Spitzer Science Center, California Institute of Technology, MS 220-6, Pasadena, CA 91125, USA \\ ${ }^{9}$ Scientific Support Office, European Space Research and Technology Centre (ESA-ESTEC/SCI-S), Keplerlaan 1, 2201 AZ Noordwijk, The Netherlands \\ ${ }^{10}$ Leiden Observatory, Leiden University, P.O. Box 9513, 2300 RA Leiden, The Netherlands \\ ${ }^{11}$ Department of Physics, University of Crete, GR-71003 Heraklion, Greece \\ ${ }^{12}$ IAASARS, National Observatory of Athens, GR-15236, Penteli, Greece \\ ${ }^{13}$ Department of Astronomy, University of Virginia, 530 McCormick Road, Charlottesville, VA 22904, USA \\ ${ }^{14}$ National Radio Astronomy Observatory, 520 Edgemont Road, Charlottesville, VA 22903, USA \\ ${ }^{15}$ Institut Ciències del Cosmos (ICCUB), Universitat de Barcelona (IEEC-UB), Martí i Franqués, 1, E-08028 Barcelona, Spain \\ ${ }^{16}$ ICREA, Pg. Lluís Companys, 23, E-08010 Barcelona, Spain \\ ${ }^{17}$ Department of Physics, University of Oxford, Denys Wilkinson Building, Keble Road, Oxford, OX1 3RH, UK \\ ${ }^{18}$ The SETI Institute, 189 Bernardo Avenue, Suite 100, Mountain View, CA 94043, USA \\ ${ }^{19}$ Gemini Observatory, Northern Operations Center, 670 N. Aohoku Place, Hilo, HI 96720, USA \\ ${ }^{20}$ Departamento de Astronomía, Universidad de Concepción, Casilla 160-C, Concepción, Chile \\ ${ }^{21}$ Pontificia Universidad Católica de Chile, Instituto de Astrofisica, Casilla 306, Santiago 22, Chile \\ ${ }^{22}$ University of Hawaii, Institute for Astronomy, 2680 Woodlawn Drive, Honolulu, HI 96822, USA \\ Received 2016 September 9; revised 2017 February 16; accepted 2017 February 22; published 2017 May 3
}

\begin{abstract}
We describe a Herschel Space Observatory 194-671 $\mu \mathrm{m}$ spectroscopic survey of a sample of 121 local luminous infrared galaxies and report the fluxes of the CO $J$ to $J-1$ rotational transitions for $4 \leqslant J \leqslant 13$, the [N II] $205 \mu \mathrm{m}$ line, the [C I] lines at 609 and $370 \mu \mathrm{m}$, as well as additional and usually fainter lines. The CO spectral line energy distributions (SLEDs) presented here are consistent with our earlier work, which was based on a smaller sample, that calls for two distinct molecular gas components in general: (i) a cold component, which emits CO lines primarily at $J \lesssim 4$ and likely represents the same gas phase traced by CO (1-0), and (ii) a warm component, which dominates over the mid- $J$ regime $(4<J \lesssim 10)$ and is intimately related to current star formation. We present evidence that the $\mathrm{CO}$ line emission associated with an active galactic nucleus is significant only at $J>10$. The flux ratios of the two [C I] lines imply modest excitation temperatures of $15-30 \mathrm{~K}$; the [C I] $370 \mu \mathrm{m}$ line scales more linearly in flux with $\mathrm{CO}(4-3)$ than with $\mathrm{CO}(7-6)$. These findings suggest that the $[\mathrm{C} \mathrm{I}]$ emission is predominantly associated with the gas component defined in (i) above. Our analysis of the stacked spectra in different far-infrared (FIR) color bins reveals an evolution of the SLED of the rotational transitions of $\mathrm{H}_{2} \mathrm{O}$ vapor as a function of the FIR color in a direction consistent with infrared photon pumping.
\end{abstract}

Key words: galaxies: active - galaxies: ISM - galaxies: star formation - infrared: galaxies - ISM: molecules submillimeter: galaxies

Supporting material: figure set, machine-readable tables

\section{Introduction}

Luminous infrared galaxies (LIRGs, defined to have an $8-1000 \mu \mathrm{m}$ total infrared luminosity $L_{\mathrm{IR}} \geqslant 10^{11} L_{\odot}$; Sanders \& Mirabel 1996), and ultra-luminous galaxies (ULIRGs, $L_{\mathrm{IR}}>10^{12} L_{\odot}$ ) dominate the cosmic star formation (SF) at $z \gtrsim 1$ (Le Flóch et al. 2005; Caputi et al. 2007; Magnelli et al. 2009, 2011; Gruppioni et al. 2013). For $z \sim 1$ up to 3, these galaxies are mixtures of two populations based on the dominant "SF mode": (i) mergers dominated by nuclear starburst with

\footnotetext{
* Based on Herschel observations. Herschel is an ESA space observatory with science instruments provided by European-led Principal Investigator consortia and with important participation from NASA.
}

warm far-infrared (FIR) colors and a high SF efficiency (SFE) similar to that of local ULIRGs, and (ii) gas-rich disk galaxies with SF extended over their disks and an SFE comparable to local spirals (e.g., Daddi et al. 2010; Genzel et al. 2010). Most ULIRGs at $z \sim 1-3$, as defined purely by their luminosities, belong to group (ii), the so-called "main-sequence" population (Elbaz et al. 2010; Muzzin et al. 2010), with FIR colors in the range occupied by typical local LIRGs (Rujopakarn et al. 2011). Due to their proximity, local LIRGs can be studied in much more detail than distant counterparts, and therefore provide valuable insights into the SF process and its interplay with dense interstellar gas in the galaxy population that dominates the cosmic SF at high redshifts. For this reason, the 
flux-limited sample of local LIRGs in the Great Observatories All-Sky LIRG Survey (GOALS; Armus et al. 2009) has been the focus of a large number of observational surveys, including imaging and/or spectroscopy in X-ray (e.g., Iwasawa et al. 2011; U et al. 2012), ultraviolet (e.g., Howell et al. 2010; Petty et al. 2014), optical/near-IR (e.g., Haan et al. 2011), mid- to far-IR (e.g., Díaz-Santos et al. 2010, 2011, 2014; Petric et al. 2011; Inami et al. 2013; Stierwalt et al. 2013, 2014), and radio continuum (e.g., Murphy et al. 2013). More recently, the GOALS sample was observed with the Herschel Space Observatory (hereafter Herschel; Pilbratt et al. 2010) in a broadband photometric survey at $70,100,160,250,350$, and $500 \mu \mathrm{m}$ (PI: D. B. Sanders; see J. Chu et al. 2017, in preparation) and a spectroscopic survey targeting some of the major FIR gas cooling lines (PI: L. Armus; see Díaz-Santos et al. 2013, 2014).

(U)LIRGs are all known to be rich in molecular gas (Sanders \& Mirabel 1996), which is the fuel necessary for their aboveaverage $\mathrm{SF}$ rates (SFRs). The $\mathrm{CO}(1-0)$ line $^{23}$ which is associated with a critical density $\left(n_{\mathrm{c}}\right)$ on the order of $10^{3} \mathrm{~cm}^{-3}$ and an excitation temperature $\left(T_{\mathrm{ex}}\right)$ of $5.5 \mathrm{~K}$, has been widely used to trace the total molecular gas content. However, SF occurs mainly in the denser parts of molecular clouds, as evidenced by correlations in local (U)LIRGs between $L_{\mathrm{IR}}$ and dense gas tracers such as $\mathrm{HCN}(1-0)$ (e.g., Gao \& Solomon 2014; Wu et al. 2005; Privon et al. 2015), and heats up the surrounding dense molecular gas substantially. The resulting warm dense gas can be better traced by a mid- $J$ CO line transition, such as $\mathrm{CO}(6-5)$, which has $n_{\mathrm{c}} \sim 10^{5} \mathrm{~cm}^{-3}$ and $T_{\mathrm{ex}}=116 \mathrm{~K}$ (Carilli \& Walter 2013). This prediction was already suggested by limited ground-based CO data (e.g., Bayet et al. 2009) prior to the advent of Herschel. In general, a CO line of a higher $J$ corresponds to higher $n_{\mathrm{c}}$ and $T_{\mathrm{ex}}$. This unique property of the $\mathrm{CO}$ rotational transitions allows one to immediately make ballpark estimates on both the gas density and temperature of the underlying molecular gas based on the $J$ value at the peak of the $\mathrm{CO}$ spectral line energy distribution (SLED) observed.

By combining our own observations with archival data, we analyzed the 194-671 $\mu \mathrm{m}$ spectra of 121 LIRGs obtained with the Fourier transform spectrometer (FTS) of the Spectral and Photometric Imaging REceiver (SPIRE; Griffin et al. 2010; Swinyard et al. 2014) on board Herschel. These galaxies belong to a complete, IR flux-limited sample of 123 LIRGs from GOALS as detailed in Section 2. One of our primary goals is to study the CO SLED in the mid- $J$ regime, i.e., $4<J \lesssim 10$, which was anticipated to be closely related to ongoing SF. Indeed, our earlier analysis of the CO SLEDs on a subset of this sample (Lu et al. 2014) suggests that a simple picture that can adequately describe the molecular gas properties in the majority of (U)LIRGs involves two gas components: (a) a cold, moderately dense gas phase, which emits $\mathrm{CO}$ lines primarily at $J<4$ and is not directly related to current SF, and (b) a warm and dense component, which emits $\mathrm{CO}$ lines mainly in the mid- $J$ regime. For the vast majority of the SF-dominated (U)LIRGs, the ratios of the total luminosity of the warm $\mathrm{CO}$ line emission to $L_{\mathrm{IR}}$ show a well defined characteristic value, suggesting strongly that current SF is the power source for both the warm $\mathrm{CO}$ and IR dust emissions in these galaxies. This

\footnotetext{
${ }^{23}$ Throughout this paper, we use $J$ to refer to the upper energy level of the $\mathrm{CO}$ rotational transition from $J$ to $J-1$. For example, CO $(1-0)$ is the rotational transition from $J=1$ to $(J-1)=0$.
}

framework was further confirmed by our high angular resolution mapping of the $\mathrm{CO}(6-5)$ line emission in some representative local LIRGs with the Atacama Large Millimeter/submillimeter Array (ALMA; Xu et al. 2014, 2015; Zhao et al. 2016b). As a result, Lu et al. (2015) analyzed the $\mathrm{CO}(7-6)$ data from the current paper and showed that a single mid- $J$ CO line, such as $\mathrm{CO}(7-6)$, can serve as a good SFR tracer for galaxies both in the local universe and at high redshifts.

As an SFR tracer, $\mathrm{CO}(7-6)$ has advantages over some conventional SFR tracers such as the luminosity of the [C II] line at $158 \mu \mathrm{m}$ and $L_{\mathrm{IR}}$. The [C II] line luminosity to $L_{\mathrm{IR}}$ (or SFR) ratio decreases steeply as the FIR color increases (e.g., Díaz-Santos et al. 2013; Lu et al. 2015). Since the FIR color is fundamentally driven by the average intensity of the dust heating radiation field (e.g., Draine \& Li 2007) and scales empirically with the average SFR surface density in disk galaxies (e.g., Liu et al. 2015; Lutz et al. 2016), this implies that, the higher the SFR surface density of a galaxy is, the less relevant (energetically) the [C II] line becomes. This runs counter to what constitutes a good SFR tracer. In contrast, the $\mathrm{CO}(7-6)$ to IR luminosity ratio depends little on the FIR color (Lu et al. 2014, 2015). $L_{\mathrm{IR}}$ is regarded as a reliable SFR tracer for active star-forming galaxies, as dust grains are very effective at absorbing far-UV photons and reradiating the energy in the infrared. For high- $z$ galaxies, however, this usually requires multiple photometric measurements covering a wide wavelength range, as illustrated in the recent studies of 3 galaxies at $z \sim 5-6$ (Riechers et al. 2013; Gilli et al. 2014; Rawle et al. 2014). Furthermore, as $z$ increases, accurate continuum photometry at submillimeter wavelengths becomes challenging due to a relatively bright background and an increasing Cosmic Microwave Background (CMB; da Cunha et al. 2013). In comparison, using CO (7-6) as the SFR tracer involves only one line flux measurement and is less impacted by $\mathrm{CMB}$, due to the high line excitation temperature. In addition, as further shown in this paper, the $\mathrm{CO}(7-6)$ line emission could also be largely free from the influence of AGNs.

In this paper we tabulate and study in more detail the SPIRE/FTS fluxes of the CO emission lines of $4 \leqslant J \leqslant 13$ for the whole sample. The $\mathrm{CO}$ data presented here can be further combined with existing ground-based $\mathrm{CO}$ lines of $1 \leqslant J \leqslant 3$ (e.g., Sanders et al. 1991; Gao \& Solomon 1999; Yao et al. 2003; Leech et al. 2010; Papadopoulos et al. 2012) to construct a "full" CO SLED that can be used to gain important insights into the physical conditions of molecular gas in (U)LIRGs and how different gas phases evolve along a merger sequence. This can by done either by modeling the observed CO SLED in a non-local thermodynamic equilibrium (non-LTE) condition, which has been applied to many individual galaxies with SPIRE/FTS data (e.g., Panuzzo et al. 2010; Van der Werf et al. 2010; Rangwala et al. 2011; Kamenetzky et al. 2012; Spinoglio et al. 2012; Meijerink et al. 2013; Pellegrini et al. 2013; Pereira-Santaella et al. 2013; Rigopoulou et al. 2013; Papadopoulos et al. 2014; Rosenberg et al. 2014a, 2014b, 2015; Schirm et al. 2014; Wu et al. 2015; Xu et al. 2015), or by empirical correlation analyses with data from other wavebands (e.g., Greve et al. 2014; Lu et al. 2014, 2015; Liu et al. 2015; Kamenetzky et al. 2016).

In addition to the $\mathrm{CO}$ lines, our other main targeted spectral lines include the fine-structure line of singly ionized nitrogen at 
$205 \mu \mathrm{m}$ (i.e., ${ }^{3} P_{1} \rightarrow{ }^{3} P_{0}$ at $1461.134 \mathrm{GHz}$; hereafter referred to as [N II] $205 \mu \mathrm{m}$ or the [N II] line) and the two fine-structure transitions of neutral carbon in its ground state at $609 \mu \mathrm{m}$ (i.e., ${ }^{3} P_{1} \rightarrow{ }^{3} P_{0}$ at $492.1607 \mathrm{GHz}$; hereafter [CI] $\left.609 \mu \mathrm{m}\right)$ and $370 \mu \mathrm{m} \quad$ (i.e., $\quad{ }^{3} P_{2} \rightarrow{ }^{3} P_{1}$ at $809.3435 \mathrm{GHz}$; hereafter [C I] $370 \mu \mathrm{m})$. Statistical analyses of our data on the [N II] line, which probes mainly low-ionization and low-density ionized gas, can be found in Zhao et al. (2013, 2016a), who also carefully derived a local luminosity function (LF) of this line. A detailed analysis of the [C $\mathrm{I}]$ line data will be presented elsewhere. The current paper describes our survey and presents the spectral lines detected. The remainder of this paper is organized as follows: we present our galaxy sample in Section 2. In Section 3 we describe our spectroscopic survey and data reduction, present the resulting spectra, and tabulate the fluxes of the detected spectral lines. In Section 4 we consider possible data systematics that may be relevant for certain future science application of the data sets given here. In Section 5 we present statistical analyses of the $\mathrm{CO}$ and [C I] lines, as well as spectral lines from $\mathrm{H}_{2} \mathrm{O}$ vapor and hydrogen fluoride (HF) molecules. Finally, in Section 6 we summarize our results.

\section{Sample}

\subsection{Sample Selection}

We selected our targets for the SPIRE/FTS survey from the GOALS sample (Armus et al. 2009). The GOALS sample consists of 202 LIRGs complete to a flux density of 5.24 Jy at $60 \mu \mathrm{m}$ as measured by the Infrared Astronomical Satellite $(I R A S)$. For a target in a multiple galaxy system, its $L_{\mathrm{IR}}$ was determined based on a flux partition between the individual galaxies at either 70 or $24 \mu \mathrm{m}$, following the scheme described in Díaz-Santos et al. (2010, 2011). Figure 1(a) is a plot of the 202 GOALS galaxies in terms of $\log L_{\mathrm{IR}}$ versus $F_{\mathrm{IR}}$, where $F_{\mathrm{IR}}$ is the $8-1000 \mu \mathrm{m}$ IR flux as defined in Sanders \& Mirabel (1996). The conversion between $F_{\mathrm{IR}}$ and $L_{\mathrm{IR}}$ was done using the luminosity distance given in Table 1 below. The horizontal dotted line stands for the $L_{\mathrm{IR}}$ cutoff for LIRGs. The vertical dotted line stands for $F_{\mathrm{IR}}=6.5 \times 10^{-13} \mathrm{~W} \mathrm{~m}^{-2}$, which was the cutoff for the initial 124 targets selected for our SPIRE/ FTS survey, including 7 ULIRGs. This flux cutoff was applied to achieve a balance between the sample size and the telescope time required to achieve our desired sensitivity. Our sample selection included one object (IRAS 05223+1908) that we now no longer consider to be an LIRG, based on the new SPIRE/ FTS data here (see Section 3.3). After excluding this source, the complete, IR flux-limited LIRG sample intended for our SPIRE/FTS survey consists of 123 sources.

While SPIRE/FTS observations of ULIRGs were also obtained by other groups, our program is the only one that provides adequate coverage of LIRGs with $L_{\mathrm{IR}}$ of $10^{11}$ to $\sim 10^{11.5} L_{\odot}$, where the LIRG population displays the largest diversity in physical properties (Armus et al. 2009). Figure 1(b) plots the FIR color, $C(60 / 100)$, defined in this work as the IRAS 60-to- $100 \mu \mathrm{m}$ flux density ratio, as a function of $F_{\mathrm{IR}}$. For a galaxy in a multiple galaxy system unresolved by IRAS, its FIR color used here is the same as that for the system as a whole, except for the cases where the 60 and $100 \mu \mathrm{m}$ flux densities of the individual galaxies were available. Figure 1(b) shows that the $C(60 / 100)$ color range covered by our FTS sample is representative of the parent sample. Figure 1(c) plots

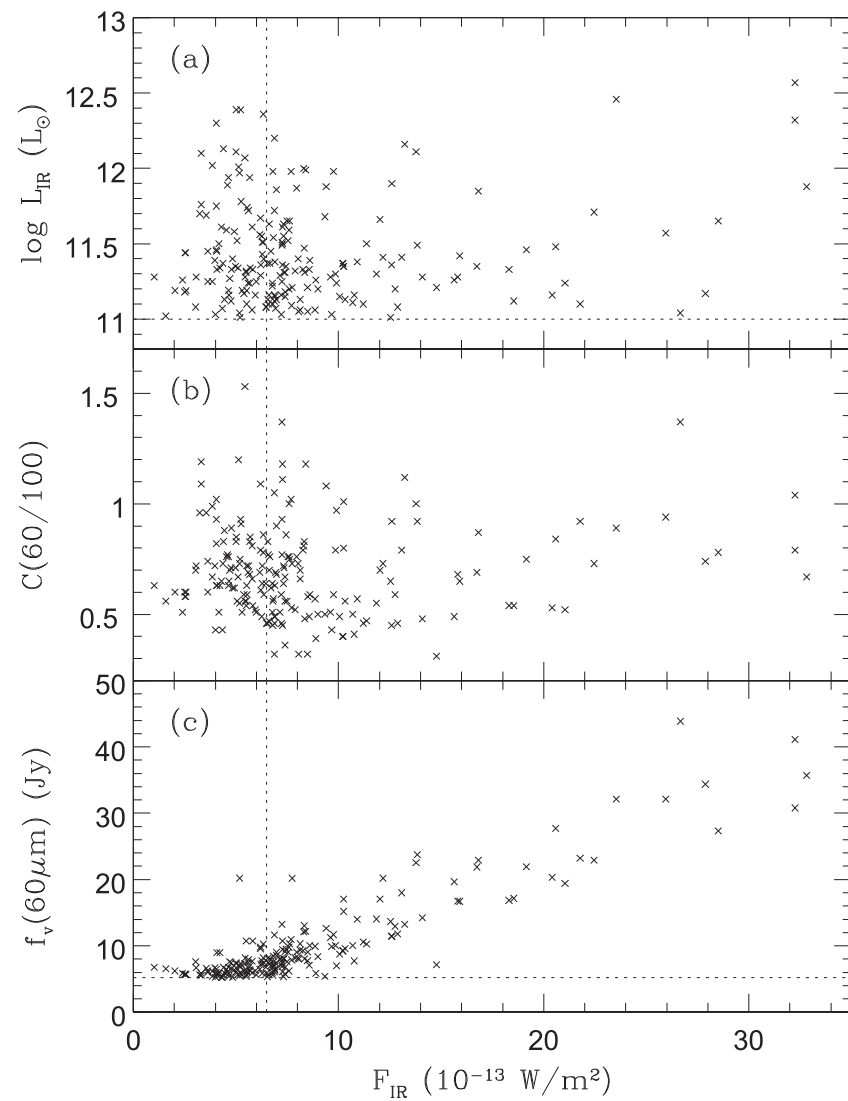

Figure 1. Plots of (a) logarithmic $L_{\mathrm{IR}}$, (b) IRAS 60-to- $100 \mu \mathrm{m}$ flux density ratio or the FIR color $C(60 / 100)$, and (c) IRAS $60 \mu \mathrm{m}$ flux density as a function of the total IR flux, $F_{\mathrm{IR}}$, for the GOALS sample of 202 LIRGs. The $L_{\mathrm{IR}}$ ad $C(60 /$ 100) are the values used at the time of the sample selection. The vertical dotted line across all the plots indicates our FTS sample selection of $F_{\mathrm{IR}}>6.5 \times 10^{-13} \mathrm{~W} \mathrm{~m}^{-2}$. The horizontal dotted lines in (a) and (c) indicate $L_{\mathrm{IR}}=10^{11} L_{\odot}$ and $f_{\nu}(60 \mu \mathrm{m})=5.24 \mathrm{Jy}$, respectively.

the IRAS $60 \mu \mathrm{m}$ flux density against $F_{\mathrm{IR}}$, with the horizontal dotted line standing for the $60 \mu \mathrm{m}$ flux density cutoff of the GOALS sample. This plot illustrates that our FTS sample is effectively limited only by our flux cutoff in $F_{\mathrm{IR}}$.

\subsection{Basic Galaxy Parameters}

Of the 123 LIRGs in our complete, IR flux-limited sample, a total of 121 were observed with SPIRE/FTS (with VV 250a and IC 4686 being the 2 objects that were not observed). In addition, we also observed two non-LIRG galaxies, NGC 5010 and the aforementioned IRAS $05223+1908$. All 123 observed targets are listed in Table 1 with the following columns: Column (1) is the name of the target spatially closest to the actual pointing of the SPIRE/FTS observation. These names follow an updated naming scheme detailed in J. M. Mazzarella et al. (2017, in preparation), with notes given in the Appendix for those galaxies with known companions. Columns (2) and (3) are the J2000 R.A. and decl. of the actual pointing of the SPIRE/FTS observation. Column (4) gives the systematic pointing offset in arcseconds, as of the calibration version 11 in the Herschel Interactive Processing Environment (HIPE; Ott 2010), between the actual pointed position and the requested pointing position. The latter is always the nuclear position of the target specified in Column (1). This pointing offset includes a 1!'7 SPIRE-specific offset applicable to the Herschel observational days (OD) earlier than OD 1110, but not any 
Table 1

SPIRE/FTS Observations

\begin{tabular}{|c|c|c|c|c|c|c|c|c|c|c|}
\hline Name & $\begin{array}{l}\text { R.A. } \\
\text { (J2000) } \\
(2)\end{array}$ & $\begin{array}{l}\text { Decl. } \\
\text { (J2000) } \\
(3)\end{array}$ & $\begin{array}{l}\delta r \\
\left({ }^{\prime \prime}\right) \\
(4)\end{array}$ & $\begin{array}{c}L_{\mathrm{IR}} \\
\left(\log L_{\odot}\right) \\
(5)\end{array}$ & $\begin{array}{c}C_{\mathrm{FIR}} \\
(6)\end{array}$ & $\begin{array}{c}D_{\text {lum }} \\
(\mathrm{Mpc}) \\
(7)\end{array}$ & $\begin{array}{c}V_{h} \\
\left(\mathrm{~km} \mathrm{~s}^{-1}\right) \\
(8)\end{array}$ & $\begin{array}{l}\text { OBSID } \\
\text { (9) }\end{array}$ & $\begin{array}{l}\text { Exp } \\
(\mathrm{sec}) \\
(10)\end{array}$ & FTS Program \\
\hline NGC 0023 & $0^{\mathrm{h}} 09^{\mathrm{m}} 53^{\mathrm{s}} \cdot 4$ & $25^{\mathrm{d}} 55^{\mathrm{m}} 26^{\mathrm{s}}$ & 0.2 & 11.11 & 0.58 & 65.2 & 4566 & 1342247622 & 1584 & OT1_nlu_1 \\
\hline NGC 0034 & $0^{\mathrm{h}} 11^{\mathrm{m}} 06^{\mathrm{s}} \cdot 6$ & $-12^{\mathrm{d}} 06^{\mathrm{m}} 24^{\mathrm{s}}$ & 2.9 & $11.49\left(^{*}\right)$ & 1.01 & 84.1 & 5881 & 1342199253 & 14832 & KPOT_pvanderw_1 \\
\hline MCG -02-01-051 & $0^{\mathrm{h}} 18^{\mathrm{m}} 50^{\mathrm{s}} .9$ & $-10^{\mathrm{d}} 22^{\mathrm{m}} 38^{\mathrm{s}}$ & 0.1 & $11.44\left(^{*}\right)$ & 0.77 & 117.5 & 8159 & 1342247617 & 2936 & OT1_nlu_1 \\
\hline ESO 350-IG038(=Haro 11) & $0^{\mathrm{h}} 36^{\mathrm{m}} 52^{\mathrm{s}} \cdot 5$ & $-33^{\mathrm{d}} 33^{\mathrm{m}} 17^{\mathrm{s}}$ & 0.0 & 11.28 & 1.37 & 89.0 & 6175 & 1342246978 & 2936 & OT1_nlu_1 \\
\hline NGC 0232 & $0^{\mathrm{h}} 42^{\mathrm{m}} 45^{\mathrm{s}} \cdot 9$ & $-23^{\mathrm{d}} 33^{\mathrm{m}} 39^{\mathrm{s}}$ & 3.1 & $11.41\left(^{*}\right)$ & 0.59 & 95.2 & 6647 & 1342221707 & 2936 & OT1_nlu_1 \\
\hline MCG +12-02-001 & $0^{\mathrm{h}} 54^{\mathrm{m}} 03^{\mathrm{s}} \cdot 4$ & $73^{\mathrm{d}} 05^{\mathrm{m}} 09^{\mathrm{s}}$ & 3.1 & 11.50 & 0.75 & 69.8 & 4706 & 1342213377 & 14428 & KPOT_pvanderw_1 \\
\hline NGC 0317B & $0^{\mathrm{h}} 57^{\mathrm{m}} 40 s 3$ & $43^{\mathrm{d}} 47^{\mathrm{m}} 33^{\mathrm{s}}$ & 0.3 & $11.18\left(^{*}\right)$ & 0.67 & 77.8 & 5429 & 1342239358 & 2936 & OT1_nlu_1 \\
\hline IC 1623 & $1^{\mathrm{h}} 07^{\mathrm{m}} 46^{\mathrm{s}} .7$ & $-17^{\mathrm{d}} 30^{\mathrm{m}} 27^{\mathrm{s}}$ & 5.3 & $11.71\left(^{*}\right)$ & 0.73 & 85.5 & 6016 & 1342212314 & 13346 & KPOT_pvanderw_1 \\
\hline MCG -03-04-014 & $1^{\mathrm{h}} 10^{\mathrm{m}} 08^{\mathrm{s}} \cdot 8$ & $-16^{\mathrm{d}} 51^{\mathrm{m}} 11^{\mathrm{s}}$ & 2.0 & 11.65 & 0.70 & 144.0 & 10040 & 1342213442 & 5640 & OT1_nlu_1 \\
\hline ESO 244-G012 & $1^{\mathrm{h}} 18^{\mathrm{m}} 08^{\mathrm{s}} \cdot 3$ & $-44^{\mathrm{d}} 27^{\mathrm{m}} 39^{\mathrm{s}}$ & 4.2 & $11.38\left(^{*}\right)$ & 0.79 & 91.5 & 6307 & 1342221708 & 2936 & OT1_nlu_1 \\
\hline CGCG 436-030 & $1^{\mathrm{h}} 20^{\mathrm{m}} 02.5$ & $14^{\mathrm{d}} 21^{\mathrm{m}} 41^{\mathrm{s}}$ & 2.2 & 11.69 & 1.11 & 134.0 & 9362 & 1342213443 & 5640 & OT1_nlu_1 \\
\hline ESO 353-G020 & $1^{\mathrm{h}} 34^{\mathrm{m}} 51^{\mathrm{s}} \cdot 3$ & $-36^{\mathrm{d}} 08^{\mathrm{m}} 15^{\mathrm{s}}$ & 0.1 & 11.06 & 0.46 & 68.8 & 4797 & 1342247615 & 2936 & OT1_nlu_1 \\
\hline III Zw 035 & $1^{\mathrm{h}} 44^{\mathrm{m}} 30.5$ & $17^{\mathrm{d}} 06^{\mathrm{m}} 09^{\mathrm{s}}$ & 0.2 & 11.64 & 0.93 & 119.0 & 8375 & 1342239343 & 2936 & OT1_nlu_1 \\
\hline NGC 0695 & $1^{\mathrm{h}} 51^{\mathrm{m}} 14^{\mathrm{s}} \cdot 4$ & $22^{\mathrm{d}} 34^{\mathrm{m}} 56^{\mathrm{s}}$ & 1.5 & 11.68 & 0.56 & 139.0 & 9735 & 1342224767 & 5640 & OT1_nlu_1 \\
\hline NGC 0828 & $2^{\mathrm{h}} 10^{\mathrm{m}} 09^{\mathrm{s}} \cdot 5$ & $39^{\mathrm{d}} 11^{\mathrm{m}} 25^{\mathrm{s}}$ & 0.2 & 11.36 & 0.45 & 76.3 & 5374 & 1342239357 & 1584 & OT1_nlu_1 \\
\hline NGC 0876 & $2^{\mathrm{h}} 18^{\mathrm{m}} 00^{\mathrm{s}} .1$ & $14^{\mathrm{d}} 32^{\mathrm{m}} 34^{\mathrm{s}}$ & 0.2 & $10.97\left(^{*}\right)$ & 0.46 & 54.6 & 3913 & 1342239342 & 1584 & OT1_nlu_1 \\
\hline UGC 01845 & $2^{\mathrm{h}} 24^{\mathrm{m}} 07^{\mathrm{s}} \cdot 9$ & $47^{\mathrm{d}} 58^{\mathrm{m}} 11^{\mathrm{s}}$ & 0.3 & 11.12 & 0.66 & 67.0 & 4679 & 1342240022 & 1584 & OT1_nlu_1 \\
\hline NGC 0958 & $2^{\mathrm{h}} 30^{\mathrm{m}} 42^{\mathrm{s}} \cdot 8$ & $-2^{\mathrm{d}} 56^{\mathrm{m}} 24^{\mathrm{s}}$ & 0.2 & 11.20 & 0.39 & 80.6 & 5738 & 1342239339 & 2936 & OT1_nlu_1 \\
\hline NGC $1068^{a}$ & $2^{\mathrm{h}} 42^{\mathrm{m}} 40^{\mathrm{s}} .8$ & $-0^{\mathrm{d}} 00^{\mathrm{m}} 48^{\mathrm{s}}$ & 2.1 & 11.40 & 0.76 & 15.9 & 1137 & 1342213445 & 5041 & KPGT_cwilso01_1 \\
\hline UGC 02238 & $2^{\mathrm{h}} 46^{\mathrm{m}} 17^{\mathrm{s}} .5$ & $13^{\mathrm{d}} 05^{\mathrm{m}} 45^{\mathrm{s}}$ & 0.1 & 11.33 & 0.52 & 92.4 & 6560 & 1342239340 & 2936 & OT1_nlu_1 \\
\hline MCG +02-08-029 & $2^{\mathrm{h}} 54^{\mathrm{m}} 01 \stackrel{\mathrm{s}}{8}$ & $14^{\mathrm{d}} 58^{\mathrm{m}} 14^{\mathrm{s}}$ & 0.1 & $11.66\left(^{*}\right)$ & 0.72 & 136.0 & 9558 & 1342239341 & 5640 & OT1_nlu_1 \\
\hline UGC $02608^{\mathrm{a}}$ & $3^{\mathrm{h}} 15^{\mathrm{m}} 01 \stackrel{\mathrm{s}}{ } \cdot 2$ & $42^{\mathrm{d}} 02^{\mathrm{m}} 09^{\mathrm{s}}$ & 0.2 & $11.38\left(^{*}\right)$ & 0.73 & 100.0 & 6998 & 1342239356 & 2936 & OT1_nlu_1 \\
\hline NGC $1275^{a}$ & $3^{\mathrm{h}} 19^{\mathrm{m}} 48^{\mathrm{s}} \cdot 2$ & $41^{\mathrm{d}} 30^{\mathrm{m}} 42^{\mathrm{s}}$ & 0.3 & 11.26 & 0.97 & 75.0 & 5264 & 1342249054 & 3612 & OT1_pogle01_1 \\
\hline UGC 02982 & $4^{\mathrm{h}} 12^{\mathrm{m}} 22^{\mathrm{s}} \cdot 5$ & $5^{\mathrm{d}} 32^{\mathrm{m}} 50^{\mathrm{s}}$ & 0.1 & 11.20 & 0.50 & 74.9 & 5305 & 1342240021 & 1584 & OT1_nlu_1 \\
\hline ESO 420-G013 & $4^{\mathrm{h}} 13^{\mathrm{m}} 49^{\mathrm{s}} \cdot 6$ & $-32^{\mathrm{d}} 00^{\mathrm{m}} 24^{\mathrm{s}}$ & 0.2 & 11.07 & 0.65 & 51.0 & 3570 & 1342242590 & 1584 & OT1_nlu_1 \\
\hline NGC 1572 & $4^{\mathrm{h}} 22^{\mathrm{m}} 42^{\mathrm{s}} \cdot 8$ & $-40^{\mathrm{d}} 36^{\mathrm{m}} 03^{\mathrm{s}}$ & 0.3 & 11.30 & 0.48 & 88.6 & 6111 & 1342242588 & 2936 & OT1_nlu_1 \\
\hline IRAS $04271+3849$ & $4^{\mathrm{h}} 30^{\mathrm{m}} 33^{\mathrm{s}} \cdot 2$ & $38^{\mathrm{d}} 55^{\mathrm{m}} 49^{\mathrm{s}}$ & 1.2 & 11.11 & 0.63 & 80.8 & 5640 & 1342227786 & 2936 & OT1_nlu_1 \\
\hline NGC 1614 & $4^{\mathrm{h}} 33^{\mathrm{m}} 59^{\mathrm{s}} .8$ & $-8^{\mathrm{d}} 34^{\mathrm{m}} 45^{\mathrm{s}}$ & 1.7 & 11.65 & 0.94 & 67.8 & 4778 & 1342192831 & 6720 & KPOT_pvanderw_1 \\
\hline UGC 03094 & $4^{\mathrm{h}} 35^{\mathrm{m}} 33^{\mathrm{s}} .8$ & $19^{\mathrm{d}} 10^{\mathrm{m}} 19^{\mathrm{s}}$ & 1.4 & 11.41 & 0.49 & 106.0 & 7408 & 1342227522 & 2936 & OT1_nlu_1 \\
\hline MCG -05-12-006 & $4^{\mathrm{h}} 52^{\mathrm{m}} 05^{\mathrm{s}} .0$ & $-32^{\mathrm{d}} 59^{\mathrm{m}} 26^{\mathrm{s}}$ & 0.2 & 11.17 & 0.83 & 81.3 & 5622 & 1342242589 & 2936 & OT1_nlu_1 \\
\hline IRAS F05189-2524 & $5^{\mathrm{h}} 21^{\mathrm{m}} 01.3$ & $-25^{\mathrm{d}} 21^{\mathrm{m}} 46^{\mathrm{s}}$ & 2.3 & 12.16 & 1.12 & 187.0 & 12760 & 1342192832 & 16996 & KPOT_pvanderw_1 \\
\hline IRAS $05223+1908^{b}$ & $5^{\mathrm{h}} 25^{\mathrm{m}} 16^{\mathrm{s}} .8$ & $19^{\mathrm{d}} 10^{\mathrm{m}} 49^{\mathrm{s}}$ & 2.4 & $\ldots . .\left({ }^{*}\right)$ & $\ldots$. & $\ldots$ & 100 & 1342228738 & 2936 & OT1_nlu_1 \\
\hline MCG +08-11-002 & $5^{\mathrm{h}} 40^{\mathrm{m}} 43^{\mathrm{s}} \cdot 8$ & $49^{\mathrm{d}} 41^{\mathrm{m}} 43^{\mathrm{s}}$ & 1.8 & 11.46 & 0.57 & 83.7 & 5743 & 1342230414 & 1584 & OT1_nlu_1 \\
\hline NGC 1961 & $5^{\mathrm{h}} 42^{\mathrm{m}} 04.7$ & $69^{\mathrm{d}} 22^{\mathrm{m}} 43^{\mathrm{s}}$ & 1.8 & 11.06 & 0.31 & 59.0 & 3934 & 1342228708 & 1584 & OT1_nlu_1 \\
\hline UGC 03351 & $5^{\mathrm{h}} 45^{\mathrm{m}} 48^{\mathrm{s}} \cdot 2$ & $58^{\mathrm{d}} 42^{\mathrm{m}} 05^{\mathrm{s}}$ & 1.8 & 11.28 & 0.48 & 65.8 & 4455 & 1342230415 & 1584 & OT1_nlu_1 \\
\hline IRAS $05442+1732$ & $5^{\mathrm{h}} 47^{\mathrm{m}} 11^{\mathrm{s}} \cdot 3$ & $17^{\mathrm{d}} 33^{\mathrm{m}} 48^{\mathrm{s}}$ & 1.8 & $11.24\left(^{*}\right)$ & 0.79 & 80.5 & 5582 & 1342230413 & 1584 & OT1_nlu_1 \\
\hline UGC 03410 & $6^{\mathrm{h}} 14^{\mathrm{m}} 30^{\mathrm{s}} .1$ & $80^{\mathrm{d}} 27^{\mathrm{m}} 01^{\mathrm{s}}$ & 1.7 & $11.02\left(^{*}\right)$ & 0.42 & 59.7 & 3921 & 1342231072 & 1584 & OT1_nlu_1 \\
\hline NGC $2146 \mathrm{NW}^{\mathrm{c}}$ & $6^{\mathrm{h}} 18^{\mathrm{m}} 36^{\mathrm{s}} .0$ & $78^{\mathrm{d}} 21^{\mathrm{m}} 32^{\mathrm{s}}$ & 2.2 & 11.12 & 0.76 & 17.5 & 893 & 1342219554 & 3070 & KPOT_pvanderw_1 \\
\hline NGC $2146 \mathrm{Nuc}^{\mathrm{c}}$ & $6^{\mathrm{h}} 18^{\mathrm{m}} 38^{\mathrm{s}} \cdot 6$ & $78^{\mathrm{d}} 21^{\mathrm{m}} 25^{\mathrm{s}}$ & 1.1 & 11.12 & 0.76 & 17.5 & 893 & 1342204025 & 3070 & KPOT_pvanderw_1 \\
\hline NGC $2146 S E^{c}$ & $6^{\mathrm{h}} 18^{\mathrm{m}} 39^{\mathrm{s}} .8$ & $78^{\mathrm{d}} 21^{\mathrm{m}} 16^{\mathrm{s}}$ & 2.2 & 11.12 & 0.76 & 17.5 & 893 & 1342219555 & 3070 & KPOT_pvanderw_1 \\
\hline ESO 255-IG 007 & $6^{\mathrm{h}} 27^{\mathrm{m}} 21.8$ & $-47^{\mathrm{d}} 10^{\mathrm{m}} 36^{\mathrm{s}}$ & 1.8 & $11.86\left(^{*}\right)$ & 0.76 & 173.0 & 11629 & 1342231084 & 5640 & OT1_nlu_1 \\
\hline UGC 03608 & $6^{\mathrm{h}} 57^{\mathrm{m}} 34.6$ & $46^{\mathrm{d}} 24^{\mathrm{m}} 12^{\mathrm{s}}$ & 2.3 & 11.34 & 0.71 & 94.3 & 6401 & 1342228744 & 2936 & OT1_nlu_1 \\
\hline NGC 2341 & $7^{\mathrm{h}} 09^{\mathrm{m}} 12^{\mathrm{s}} \cdot 2$ & $20^{\mathrm{d}} 36^{\mathrm{m}} 13^{\mathrm{s}}$ & 1.5 & $10.97\left(^{*}\right)$ & 0.77 & 78.0 & 5276 & 1342228730 & 2936 & OT1_nlu_1 \\
\hline NGC 2342 & $7^{\mathrm{h}} 09^{\mathrm{m}} 18^{\mathrm{s}} \cdot 1$ & $20^{\mathrm{d}} 38^{\mathrm{m}} 10^{\mathrm{s}}$ & 1.5 & $11.03\left(^{*}\right)$ & 0.48 & 78.0 & 5276 & 1342228729 & 2936 & OT1_nlu_1 \\
\hline NGC 2369 & $7^{\mathrm{h}} 16^{\mathrm{m}} 37^{\mathrm{s}} .8$ & $-62^{\mathrm{d}} 20^{\mathrm{m}} 35^{\mathrm{s}}$ & 1.8 & 11.16 & 0.53 & 47.6 & 3240 & 1342231083 & 1584 & OT1_nlu_1 \\
\hline NGC 2388 & $7^{\mathrm{h}} 28^{\mathrm{m}} 53^{\mathrm{s}} \cdot 6$ & $33^{\mathrm{d}} 49^{\mathrm{m}} 09^{\mathrm{s}}$ & 1.8 & $11.28\left(^{*}\right)$ & 0.68 & 62.1 & 4134 & 1342231071 & 1584 & OT1_nlu_1 \\
\hline MCG +02-20-003 & $7^{\mathrm{h}} 35^{\mathrm{m}} 43^{\mathrm{s}} \cdot 5$ & $11^{\mathrm{d}} 42^{\mathrm{m}} 36^{\mathrm{s}}$ & 1.3 & 11.13 & 0.70 & 72.8 & 4873 & 1342228728 & 2936 & OT1_nlu_1 \\
\hline IRAS 08355-4944 & $8^{\mathrm{h}} 37^{\mathrm{m}} 02^{\mathrm{s}} \cdot 0$ & $-49^{\mathrm{d}} 54^{\mathrm{m}} 29^{\mathrm{s}}$ & 1.8 & 11.62 & 1.18 & 118.0 & 7764 & 1342231975 & 2936 & OT1_nlu_1 \\
\hline NGC 2623 & $8^{\mathrm{h}} 38^{\mathrm{m}} 24^{\mathrm{s}} \cdot 0$ & $25^{\mathrm{d}} 45^{\mathrm{m}} 17^{\mathrm{s}}$ & 0.8 & 11.60 & 0.92 & 84.1 & 5549 & 1342219553 & 12534 & KPOT_pvanderw_1 \\
\hline IRAS 09022-3615 & $9^{\mathrm{h}} 04^{\mathrm{m}} 12^{\mathrm{s}} .8$ & $-36^{\mathrm{d}} 27^{\mathrm{m}} 00^{\mathrm{s}}$ & 1.9 & 12.31 & 1.05 & 271.0 & 17880 & 1342231063 & 8344 & OT1_nlu_1 \\
\hline UGC 05101 & $9^{\mathrm{h}} 35^{\mathrm{m}} 51^{\mathrm{s}} \cdot 9$ & $61^{\mathrm{d}} 21^{\mathrm{m}} 11^{\mathrm{s}}$ & 2.1 & 12.01 & 0.59 & 177.0 & 11802 & 1342209278 & 5098 & GT1_lspinogl_2 \\
\hline NGC 3110 & $10^{\mathrm{h}} 04^{\mathrm{m}} 02^{\mathrm{s}} .2$ & $-6^{\mathrm{d}} 28^{\mathrm{m}} 28^{\mathrm{s}}$ & 1.7 & 11.37 & 0.51 & 79.5 & 5054 & 1342231971 & 1584 & OT1_nlu_1 \\
\hline NGC 3221 & $10^{\mathrm{h}} 22^{\mathrm{m}} 20^{\mathrm{s}} \cdot 4$ & $21^{\mathrm{d}} 34^{\mathrm{m}} 21^{\mathrm{s}}$ & 3.1 & 11.09 & 0.41 & 65.7 & 4110 & 1342221714 & 1584 & OT1_nlu_1 \\
\hline NGC 3256 & $10^{\mathrm{h}} 27^{\mathrm{m}} 51^{\mathrm{s}} \cdot 3$ & $-43^{\mathrm{d}} 54^{\mathrm{m}} 15^{\mathrm{s}}$ & 1.7 & 11.64 & 0.90 & 38.9 & 2804 & 1342201201 & 5234 & KPOT_pvanderw_1 \\
\hline ESO 264-G036 & $10^{\mathrm{h}} 43^{\mathrm{m}} 07^{\mathrm{s}} .7$ & $-46^{\mathrm{d}} 12^{\mathrm{m}} 45^{\mathrm{s}}$ & 0.3 & 11.32 & 0.45 & 100.0 & 6299 & 1342249044 & 2936 & OT1_nlu_1 \\
\hline ESO 264-G057 & $10^{\mathrm{h}} 59^{\mathrm{m}} 01^{\mathrm{s}} .8$ & $-43^{\mathrm{d}} 26^{\mathrm{m}} 26^{\mathrm{s}}$ & 0.3 & 11.14 & 0.47 & 83.3 & 5156 & 1342249043 & 2936 & OT1_nlu_1 \\
\hline IRAS F10565+2448 & $10^{\mathrm{h}} 59^{\mathrm{m}} 18^{\mathrm{s}} \cdot 2$ & $24^{\mathrm{d}} 32^{\mathrm{m}} 34^{\mathrm{s}}$ & 0.0 & 12.08 & 0.81 & 197.0 & 12921 & 1342247096 & 5640 & OT1_nlu_1 \\
\hline UGC $06471^{\mathrm{d}}$ & $11^{\mathrm{h}} 28^{\mathrm{m}} 30^{\mathrm{s}} \cdot 6$ & $58^{\mathrm{d}} 33^{\mathrm{m}} 39^{\mathrm{s}}$ & 3.6 & 11.93 & 1.01 & 50.7 & 3093 & 1342199249 & 4964 & KPOT_pvanderw_1 \\
\hline NGC $3690^{\mathrm{d}}$ & $11^{\mathrm{h}} 28^{\mathrm{m}} 30^{\mathrm{s}} \cdot 6$ & $58^{\mathrm{d}} 33^{\mathrm{m}} 48^{\mathrm{s}}$ & 3.6 & 11.93 & 1.01 & 50.7 & 3093 & 1342199250 & 4964 & KPOT_pvanderw_1 \\
\hline UGC $06472^{\mathrm{d}}$ & $11^{\mathrm{h}} 28^{\mathrm{m}} 33^{\mathrm{s}} \cdot 2$ & $58^{\mathrm{d}} 33^{\mathrm{m}} 45^{\mathrm{s}}$ & 3.6 & 11.93 & 1.01 & 50.7 & 3093 & 1342199248 & 4964 & KPOT_pvanderw_1 \\
\hline ESO 320-G030 & $11^{\mathrm{h}} 53^{\mathrm{m}} 11^{\mathrm{s}} .7$ & $-39^{\mathrm{d}} 07^{\mathrm{m}} 50^{\mathrm{s}}$ & 1.0 & 11.17 & 0.74 & 41.2 & 3232 & 1342210861 & 6044 & KPOT_pvanderw_1 \\
\hline NGC 4194 & $12^{\mathrm{h}} 14^{\mathrm{m}} 09^{\mathrm{s}} .8$ & $54^{\mathrm{d}} 31^{\mathrm{m}} 35^{\mathrm{s}}$ & 1.7 & 11.10 & 0.92 & 43.0 & 2501 & 1342231069 & 1584 & OT1_nlu_1 \\
\hline
\end{tabular}


Table 1

(Continued)

\begin{tabular}{|c|c|c|c|c|c|c|c|c|c|c|}
\hline Name & $\begin{array}{l}\text { R.A. } \\
\text { (J2000) } \\
(2)\end{array}$ & $\begin{array}{l}\text { Decl. } \\
\text { (J2000) } \\
(3)\end{array}$ & $\begin{array}{l}\delta r \\
\left({ }^{\prime \prime}\right) \\
(4)\end{array}$ & $\begin{array}{c}L_{\mathrm{IR}} \\
\left(\log L_{\odot}\right) \\
(5)\end{array}$ & $\begin{array}{l}C_{\mathrm{FIR}} \\
(6)\end{array}$ & $\begin{array}{c}D_{\text {lum }} \\
(\mathrm{Mpc}) \\
(7)\end{array}$ & $\begin{array}{c}V_{h} \\
\left(\mathrm{~km} \mathrm{~s}^{-1}\right) \\
(8)\end{array}$ & $\begin{array}{c}\text { OBSID } \\
\text { (9) }\end{array}$ & $\begin{array}{l}\text { Exp } \\
(\mathrm{sec}) \\
(10)\end{array}$ & FTS Program \\
\hline IRAS $12116-5615$ & $12^{\mathrm{h}} 14^{\mathrm{m}} 22^{\mathrm{s}} \cdot 1$ & $-56^{\mathrm{d}} 32^{\mathrm{m}} 33^{\mathrm{s}}$ & 0.3 & 11.65 & 0.76 & 128.0 & 8125 & 1342249462 & 5640 & OT1_nlu_1 \\
\hline NGC 4418 & $12^{\mathrm{h}} 26^{\mathrm{m}} 54^{\mathrm{s}} \cdot 6$ & $-0^{\mathrm{d}} 52^{\mathrm{m}} 39^{\mathrm{s}}$ & 0.0 & 11.19 & 1.37 & 36.5 & 2179 & 1342210848 & 9491 & KPGT_esturm_1 \\
\hline UGC $08058^{\mathrm{a}}(=$ Mrk 231) & $12^{\mathrm{h}} 56^{\mathrm{m}} 14^{\mathrm{s}} .5$ & $56^{\mathrm{d}} 52^{\mathrm{m}} 26^{\mathrm{s}}$ & 2.4 & 12.57 & 1.04 & 192.0 & 12642 & 1342210493 & 14292 & KPOT_pvanderw_1 \\
\hline MCG -02-33-098 & $13^{\mathrm{h}} 02^{\mathrm{m}} 19^{\mathrm{s}} .8$ & $-15^{\mathrm{d}} 46^{\mathrm{m}} 04^{\mathrm{s}}$ & 0.1 & $11.16\left(^{*}\right)$ & 0.77 & 78.7 & 4773 & 1342247567 & 2936 & OT1_nlu_1 \\
\hline ESO 507-G070 & $13^{\mathrm{h}} 02^{\mathrm{m}} 52^{\mathrm{s}} \cdot 3$ & $-23^{\mathrm{d}} 55^{\mathrm{m}} 18^{\mathrm{s}}$ & 0.1 & 11.56 & 0.83 & 106.0 & 6506 & 1342248421 & 2936 & OT1_nlu_1 \\
\hline NGC $5010^{\mathrm{e}}$ & $13^{\mathrm{h}} 12^{\mathrm{m}} 26^{\mathrm{s}} .5$ & $-15^{\mathrm{d}} 47^{\mathrm{m}} 51^{\mathrm{s}}$ & 1.6 & 10.82 & 0.47 & 44.4 & 2975 & 1342236996 & 1584 & OT1_nlu_1 \\
\hline IRAS $13120-5453$ & $13^{\mathrm{h}} 15^{\mathrm{m}} 06^{\mathrm{s}} .3$ & $-55^{\mathrm{d}} 09^{\mathrm{m}} 23^{\mathrm{s}}$ & 0.5 & 12.32 & 0.79 & 144.0 & 9222 & 1342212342 & 4152 & KPOT_pvanderw_1 \\
\hline UGC 08387 & $13^{\mathrm{h}} 20^{\mathrm{m}} 35^{\mathrm{s}} .5$ & $34^{\mathrm{d}} 08^{\mathrm{m}} 23^{\mathrm{s}}$ & 1.9 & 11.73 & 0.70 & 110.0 & 6985 & 1342209853 & 14832 & KPOT_pvanderw_1 \\
\hline NGC 5104 & $13^{\mathrm{h}} 21^{\mathrm{m}} 23^{\mathrm{s}} .1$ & $0^{\mathrm{d}} 20^{\mathrm{m}} 33^{\mathrm{s}}$ & 0.1 & 11.27 & 0.51 & 90.8 & 5578 & 1342247566 & 2936 & OT1_nlu_1 \\
\hline MCG -03-34-064 & $13^{\mathrm{h}} 22^{\mathrm{m}} 24^{\mathrm{s}} \cdot 4$ & $-16^{\mathrm{d}} 43^{\mathrm{m}} 43^{\mathrm{s}}$ & 0.1 & 11.17 & 1.00 & 82.2 & 4959 & 1342249041 & 2936 & OT1_nlu_1 \\
\hline NGC 5135 & $13^{\mathrm{h}} 25^{\mathrm{m}} 44^{\mathrm{s}} .0$ & $-29^{\mathrm{d}} 49^{\mathrm{m}} 60^{\mathrm{s}}$ & 1.4 & 11.30 & 0.54 & 60.9 & 4105 & 1342212344 & 14832 & KPOT_pvanderw_1 \\
\hline ESO 173-G015 & $13^{\mathrm{h}} 27^{\mathrm{m}} 23^{\mathrm{s}} .7$ & $-57^{\mathrm{d}} 29^{\mathrm{m}} 23^{\mathrm{s}}$ & 0.8 & 11.38 & 0.81 & 34.0 & 2918 & 1342202268 & 1988 & KPOT_pvanderw_1 \\
\hline IC 4280 & $13^{\mathrm{h}} 32^{\mathrm{m}} 53^{\mathrm{s}} .3$ & $-24^{\mathrm{d}} 12^{\mathrm{m}} 26^{\mathrm{s}}$ & 0.1 & 11.15 & 0.49 & 82.4 & 4889 & 1342249042 & 2936 & OT1_nlu_1 \\
\hline UGC 08696 & $13^{\mathrm{h}} 44^{\mathrm{m}} 42^{\mathrm{s}} \cdot 3$ & $55^{\mathrm{d}} 53^{\mathrm{m}} 10^{\mathrm{s}}$ & 3.3 & 12.21 & 1.00 & 173.0 & 11326 & 1342209850 & 13616 & KPOT_pvanderw_1 \\
\hline UGC 08739 & $13^{\mathrm{h}} 49^{\mathrm{m}} 14^{\mathrm{s}} .3$ & $35^{\mathrm{d}} 15^{\mathrm{m}} 20^{\mathrm{s}}$ & 0.0 & 11.15 & 0.36 & 81.4 & 5032 & 1342247123 & 2936 & OT1_nlu_1 \\
\hline ESO 221-IG 010 & $13^{\mathrm{h}} 50^{\mathrm{m}} 56^{\mathrm{s}} .9$ & $-49^{\mathrm{d}} 03^{\mathrm{m}} 19^{\mathrm{s}}$ & 0.2 & 11.22 & 0.59 & 62.9 & 3099 & 1342249461 & 1584 & OT1_nlu_1 \\
\hline NGC 5653 & $14^{\mathrm{h}} 30^{\mathrm{m}} 09^{\mathrm{s}} .9$ & $31^{\mathrm{d}} 12^{\mathrm{m}} 56^{\mathrm{s}}$ & 0.1 & $11.13\left(^{*}\right)$ & 0.46 & 60.2 & 3562 & 1342247565 & 1584 & OT1_nlu_1 \\
\hline NGC 5734 & $14^{\mathrm{h}} 45^{\mathrm{m}} 09^{\mathrm{s}} \cdot 0$ & $-20^{\mathrm{d}} 52^{\mathrm{m}} 13^{\mathrm{s}}$ & 0.1 & $10.99\left(^{*}\right)$ & 0.46 & 67.1 & 4121 & 1342248417 & 1584 & OT1_nlu_1 \\
\hline VV $340 a$ & $14^{\mathrm{h}} 57^{\mathrm{m}} 00^{\mathrm{s}} .8$ & $24^{\mathrm{d}} 37^{\mathrm{m}} 05^{\mathrm{s}}$ & 1.5 & $11.66\left(^{*}\right)$ & 0.46 & 157.0 & 10094 & 1342238241 & 5640 & OT1_nlu_1 \\
\hline IC $4518 \mathrm{~A}$ & $14^{\mathrm{h}} 57^{\mathrm{m}} 41^{\mathrm{s}} \cdot 1$ & $-43^{\mathrm{d}} 07^{\mathrm{m}} 56^{\mathrm{s}}$ & 0.1 & $11.16\left(^{*}\right)$ & 0.59 & 80.0 & 4763 & 1342250514 & 2936 & OT1_nlu_1 \\
\hline CGCG 049-057 & $15^{\mathrm{h}} 13^{\mathrm{m}} 13^{\mathrm{s}} \cdot 2$ & $7^{\mathrm{d}} 13^{\mathrm{m}} 29^{\mathrm{s}}$ & 3.2 & 11.35 & 0.69 & 65.4 & 3897 & 1342212346 & 14832 & KPOT_pvanderw_1 \\
\hline VV 705 & $15^{\mathrm{h}} 18^{\mathrm{m}} 06^{\mathrm{s}} \cdot 3$ & $42^{\mathrm{d}} 44^{\mathrm{m}} 45^{\mathrm{s}}$ & 1.5 & $11.92\left(^{*}\right)$ & 0.90 & 183.0 & 11944 & 1342238712 & 5640 & OT1_nlu_1 \\
\hline ESO 099-G004 & $15^{\mathrm{h}} 24^{\mathrm{m}} 57^{\mathrm{s}} .7$ & $-63^{\mathrm{d}} 07^{\mathrm{m}} 30^{\mathrm{s}}$ & 1.8 & 11.74 & 0.75 & 137.0 & 8779 & 1342230419 & 5640 & OT1_nlu_1 \\
\hline NGC 5936 & $15^{\mathrm{h}} 30^{\mathrm{m}} 00^{\mathrm{s}} \cdot 8$ & $12^{\mathrm{d}} 59^{\mathrm{m}} 22^{\mathrm{s}}$ & 0.2 & 11.14 & 0.49 & 67.1 & 4004 & 1342249046 & 1584 & OT1_nlu_1 \\
\hline UGC 09913 (=Arp 220) & $15^{\mathrm{h}} 34^{\mathrm{m}} 57^{\mathrm{s}} .2$ & $23^{\mathrm{d}} 30^{\mathrm{m}} 12^{\mathrm{s}}$ & 1.5 & 12.27 & 0.90 & 87.9 & 5434 & 1342190674 & 10445 & KPGT_cwilso01_1 \\
\hline NGC 5990 & $15^{\mathrm{h}} 46^{\mathrm{m}} 16^{\mathrm{s}} .4$ & $2^{\mathrm{d}} 24^{\mathrm{m}} 55^{\mathrm{s}}$ & 0.2 & 11.13 & 0.56 & 64.4 & 3839 & 1342240016 & 1584 & OT1_nlu_1 \\
\hline NGC 6052 & $16^{\mathrm{h}} 05^{\mathrm{m}} 12^{\mathrm{s}} .9$ & $20^{\mathrm{d}} 32^{\mathrm{m}} 37^{\mathrm{s}}$ & 0.2 & 11.09 & 0.64 & 77.6 & 4739 & 1342212347 & 2936 & OT1_nlu_1 \\
\hline CGCG 052-037 & $16^{\mathrm{h}} 30^{\mathrm{m}} 56^{\mathrm{s}} \cdot 6$ & $4^{\mathrm{d}} 04^{\mathrm{m}} 59^{\mathrm{s}}$ & 0.2 & $11.43\left(^{*}\right)$ & 0.62 & 116.0 & 7342 & 1342251284 & 5640 & OT1_nlu_1 \\
\hline NGC 6156 & $16^{\mathrm{h}} 34^{\mathrm{m}} 52^{\mathrm{s}} \cdot 3$ & $-60^{\mathrm{d}} 37^{\mathrm{m}} 08^{\mathrm{s}}$ & 1.8 & 11.14 & 0.54 & 48.0 & 3263 & 1342231041 & 1584 & OT1_nlu_1 \\
\hline 2MASX J16381190-6826080 & $16^{\mathrm{h}} 38^{\mathrm{m}} 11^{\mathrm{s}} .5$ & $-68^{\mathrm{d}} 26^{\mathrm{m}} 08^{\mathrm{s}}$ & 1.8 & $11.97\left(^{*}\right)$ & 0.56 & 212.0 & 139 & 1342231040 & 8344 & OT1_nlu_1 \\
\hline IRAS F16399-0937 & $16^{\mathrm{h}} 42^{\mathrm{m}} 40^{\mathrm{s}} .1$ & $-9^{\mathrm{d}} 43^{\mathrm{m}} 13^{\mathrm{s}}$ & 0.2 & 11.63 & 0.57 & 128.0 & 8098 & 1342251334 & 5640 & OT1_nlu_1 \\
\hline NGC 6240 & $16^{\mathrm{h}} 52^{\mathrm{m}} 59^{\mathrm{s}} .1$ & $2^{\mathrm{d}} 24^{\mathrm{m}} 04^{\mathrm{s}}$ & 3.5 & 11.93 & 0.87 & 116.0 & 7339 & 1342214831 & 13346 & KPOT_pvanderw_1 \\
\hline IRAS F16516-0948 & $16^{\mathrm{h}} 54^{\mathrm{m}} 23^{\mathrm{s}} .8$ & $-9^{\mathrm{d}} 53^{\mathrm{m}} 21^{\mathrm{s}}$ & 0.2 & 11.31 & 0.46 & 107.0 & 6755 & 1342251335 & 5640 & OT1_nlu_1 \\
\hline NGC 6285 & $16^{\mathrm{h}} 58^{\mathrm{m}} 23^{\mathrm{s}} .9$ & $58^{\mathrm{d}} 57^{\mathrm{m}} 19^{\mathrm{s}}$ & 1.7 & $10.85\left(^{*}\right)$ & 0.39 & 85.7 & 5501 & 1342231068 & 1584 & OT1_nlu_1 \\
\hline NGC 6286 & $16^{\mathrm{h}} 58^{\mathrm{m}} 31^{\mathrm{s}} .4$ & $58^{\mathrm{d}} 56^{\mathrm{m}} 17^{\mathrm{s}}$ & 5.4 & $\left.11.29^{*}\right)$ & 0.44 & 85.7 & 5501 & 1342221715 & 1584 & OT1_nlu_1 \\
\hline IRAS F17138-1017 & $17^{\mathrm{h}} 16^{\mathrm{m}} 35^{\mathrm{s}} .7$ & $-10^{\mathrm{d}} 20^{\mathrm{m}} 42^{\mathrm{s}}$ & 1.7 & 11.49 & 0.80 & 84.0 & 5197 & 1342230418 & 1584 & OT1_nlu_1 \\
\hline IRAS F17207-0014 & $17^{\mathrm{h}} 23^{\mathrm{m}} 22^{\mathrm{s}} \cdot 0$ & $-0^{\mathrm{d}} 17^{\mathrm{m}} 00^{\mathrm{s}}$ & 1.3 & 12.46 & 0.89 & 198.0 & 12834 & 1342192829 & 6720 & KPOT_pvanderw_1 \\
\hline ESO 138-G027 & $17^{\mathrm{h}} 26^{\mathrm{m}} 43^{\mathrm{s}} \cdot 1$ & $-59^{\mathrm{d}} 55^{\mathrm{m}} 56^{\mathrm{s}}$ & 1.8 & 11.41 & 0.86 & 98.3 & 6230 & 1342231042 & 2936 & OT1_nlu_1 \\
\hline UGC 11041 & $17^{\mathrm{h}} 54^{\mathrm{m}} 51^{\mathrm{s}} .7$ & $34^{\mathrm{d}} 46^{\mathrm{m}} 33^{\mathrm{s}}$ & 1.7 & 11.11 & 0.46 & 77.5 & 4881 & 1342231061 & 2936 & OT1_nlu_1 \\
\hline IRAS $17578-0400$ & $18^{\mathrm{h}} 00^{\mathrm{m}} 31^{\mathrm{s}} .8$ & $-4^{\mathrm{d}} 00^{\mathrm{m}} 54^{\mathrm{s}}$ & 1.7 & $11.39\left(^{*}\right)$ & 0.84 & 68.5 & 4210 & 1342231047 & 1584 & OT1_nlu_1 \\
\hline NGC 6621 & $18^{\mathrm{h}} 12^{\mathrm{m}} 55^{\mathrm{s}} .1$ & $68^{\mathrm{d}} 21^{\mathrm{m}} 46^{\mathrm{s}}$ & 1.2 & $11.27\left(^{*}\right)$ & 0.56 & 94.3 & 6191 & 1342221716 & 2936 & OT1_nlu_1 \\
\hline IC 4687 & $18^{\mathrm{h}} 13^{\mathrm{m}} 39^{\mathrm{s}} .7$ & $-57^{\mathrm{d}} 43^{\mathrm{m}} 30^{\mathrm{s}}$ & 1.2 & $11.36\left(^{*}\right)$ & 0.73 & 81.9 & 5200 & 1342192993 & 14562 & KPOT_pvanderw_1 \\
\hline IRAS F18293-3413 & $18^{\mathrm{h}} 32^{\mathrm{m}} 41^{\mathrm{s}} \cdot 3$ & $-34^{\mathrm{d}} 11^{\mathrm{m}} 26^{\mathrm{s}}$ & 2.3 & 11.88 & 0.67 & 86.0 & 5449 & 1342192830 & 5640 & KPOT_pvanderw_1 \\
\hline IC 4734 & $18^{\mathrm{h}} 38^{\mathrm{m}} 25^{\mathrm{s}} \cdot 6$ & $-57^{\mathrm{d}} 29^{\mathrm{m}} 25^{\mathrm{s}}$ & 0.2 & 11.35 & 0.55 & 73.4 & 4680 & 1342240013 & 1584 & OT1_nlu_1 \\
\hline NGC 6701 & $18^{\mathrm{h}} 43^{\mathrm{m}} 12^{\mathrm{s}} .5$ & $60^{\mathrm{d}} 39^{\mathrm{m}} 10^{\mathrm{s}}$ & 1.7 & 11.12 & 0.50 & 62.4 & 3965 & 1342231994 & 1584 & OT1_nlu_1 \\
\hline ESO 339-G011 & $19^{\mathrm{h}} 57^{\mathrm{m}} 37^{\mathrm{s}} .5$ & $-37^{\mathrm{d}} 56^{\mathrm{m}} 10^{\mathrm{s}}$ & 1.7 & 11.20 & 0.64 & 88.6 & 5756 & 1342231990 & 2936 & OT1_nlu_1 \\
\hline MCG +04-48-002 & $20^{\mathrm{h}} 28^{\mathrm{m}} 35^{\mathrm{s}} .1$ & $25^{\mathrm{d}} 44^{\mathrm{m}} 03 \mathrm{~s}$ & 2.9 & $11.00\left(^{*}\right)$ & 0.57 & 64.2 & 4167 & 1342221682 & 1584 & OT1_nlu_1 \\
\hline NGC 6926 & $20^{\mathrm{h}} 33^{\mathrm{m}} 06^{\mathrm{s}} .0$ & $-2^{\mathrm{d}} 01^{\mathrm{m}} 40^{\mathrm{s}}$ & 1.7 & 11.32 & 0.49 & 89.1 & 5880 & 1342231050 & 2936 & OT1_nlu_1 \\
\hline CGCG 448-020 & $20^{\mathrm{h}} 57^{\mathrm{m}} 24^{\mathrm{s}} .4$ & $17^{\mathrm{d}} 07^{\mathrm{m}} 38^{\mathrm{s}}$ & 1.4 & $11.94\left(^{*}\right)$ & 1.08 & 161.0 & 10822 & 1342221679 & 2936 & OT1_nlu_1 \\
\hline ESO 286-IG 019 & $20^{\mathrm{h}} 58^{\mathrm{m}} 26^{\mathrm{s}} .8$ & $-42^{\mathrm{d}} 39^{\mathrm{m}} 01^{\mathrm{s}}$ & 0.0 & 12.06 & 1.18 & 193.0 & 12890 & 1342245107 & 5640 & OT1_nlu_1 \\
\hline ESO 286-G035 & $21^{\mathrm{h}} 04^{\mathrm{m}} 11^{\mathrm{s}} \cdot 2$ & $-43^{\mathrm{d}} 35^{\mathrm{m}} 31^{\mathrm{s}}$ & 3.1 & 11.20 & 0.68 & 79.1 & 5205 & 1342216901 & 2936 & OT1_nlu_1 \\
\hline NGC 7130 & $21^{\mathrm{h}} 48^{\mathrm{m}} 19^{\mathrm{s}} \cdot 4$ & $-34^{\mathrm{d}} 57^{\mathrm{m}} 04^{\mathrm{s}}$ & 1.5 & 11.42 & 0.65 & 72.7 & 4842 & 1342219565 & 5098 & GT1_lspinog1_2 \\
\hline ESO 467-G027 & $22^{\mathrm{h}} 14^{\mathrm{m}} 39^{\mathrm{s}} .8$ & $-27^{\mathrm{d}} 27^{\mathrm{m}} 50^{\mathrm{s}}$ & 0.1 & 11.08 & 0.45 & 77.3 & 5217 & 1342245108 & 2936 & OT1_nlu_1 \\
\hline IC 5179 & $22^{\mathrm{h}} 16^{\mathrm{m}} 09^{\mathrm{s}} .1$ & $-36^{\mathrm{d}} 50^{\mathrm{m}} 37^{\mathrm{s}}$ & 0.1 & 11.24 & 0.52 & 51.4 & 3422 & 1342245109 & 1584 & OT1_nlu_1 \\
\hline UGC 12150 & $22^{\mathrm{h}} 41^{\mathrm{m}} 12^{\mathrm{s}} \cdot 3$ & $34^{\mathrm{d}} 14^{\mathrm{m}} 56^{\mathrm{s}}$ & 0.9 & 11.35 & 0.51 & 93.5 & 6413 & 1342221699 & 2936 & OT1_nlu_1 \\
\hline NGC 7469 & $23^{\mathrm{h}} 03^{\mathrm{m}} 15^{\mathrm{s}} .9$ & $8^{\mathrm{d}} 52^{\mathrm{m}} 30^{\mathrm{s}}$ & 5.7 & $11.58\left(^{*}\right)$ & 0.78 & 70.8 & 4892 & 1342199252 & 12400 & KPOT_pvanderw_1 \\
\hline ESO 148-IG 002 & $23^{\mathrm{h}} 15^{\mathrm{m}} 46^{\mathrm{s}} .7$ & $-59^{\mathrm{d}} 03^{\mathrm{m}} 15^{\mathrm{s}}$ & 0.0 & 12.06 & 1.02 & 199.0 & 13371 & 1342245110 & 7396 & OT1_nlu_1 \\
\hline IC 5298 & $23^{\mathrm{h}} 16^{\mathrm{m}} 00^{\mathrm{s}} \cdot 9$ & $25^{\mathrm{d}} 33^{\mathrm{m}} 26^{\mathrm{s}}$ & 3.6 & 11.60 & 0.76 & 119.0 & 8221 & 1342221700 & 2936 & OT1_nlu_1 \\
\hline NGC 7552 & $23^{\mathrm{h}} 16^{\mathrm{m}} 10^{\mathrm{s}} .8$ & $-42^{\mathrm{d}} 35^{\mathrm{m}} 05^{\mathrm{s}}$ & 1.0 & 11.11 & 0.75 & 23.5 & 1608 & 1342198428 & 1988 & KPOT_pvanderw_1 \\
\hline NGC 7591 & $23^{\mathrm{h}} 18^{\mathrm{m}} 16^{\mathrm{s}} \cdot 3$ & $6^{\mathrm{d}} 35^{\mathrm{m}} 09^{\mathrm{s}}$ & 0.0 & 11.12 & 0.53 & 71.4 & 4956 & 1342257346 & 2936 & OT1_nlu_1 \\
\hline NGC 7592 & $23^{\mathrm{h}} 18^{\mathrm{m}} 22 \mathrm{~s} \cdot 3$ & $-4^{\mathrm{d}} 24^{\mathrm{m}} 57^{\mathrm{s}}$ & 2.7 & $11.40\left(^{*}\right)$ & 0.76 & 106.0 & 7328 & 1342221702 & 2936 & OT1_nlu_1 \\
\hline NGC $7674^{\mathrm{a}}$ & $23^{\mathrm{h}} 27^{\mathrm{m}} 56^{\mathrm{s}} .7$ & $8^{\mathrm{d}} 46^{\mathrm{m}} 44^{\mathrm{s}}$ & 0.0 & $11.54\left(^{*}\right)$ & 0.64 & 125.0 & 8671 & 1342245858 & 5640 & OT1_nlu_1 \\
\hline
\end{tabular}


Table 1

(Continued)

\begin{tabular}{|c|c|c|c|c|c|c|c|c|c|c|}
\hline Name & $\begin{array}{l}\text { R.A. } \\
\text { (J2000) } \\
(2)\end{array}$ & $\begin{array}{c}\text { Decl. } \\
(\mathrm{J} 2000) \\
(3)\end{array}$ & $\begin{array}{l}\delta r \\
\left({ }^{\prime \prime}\right) \\
(4)\end{array}$ & $\begin{array}{c}L_{\mathrm{IR}} \\
\left(\log L_{\odot}\right) \\
(5)\end{array}$ & $\begin{array}{l}C_{\mathrm{FIR}} \\
(6)\end{array}$ & $\begin{array}{c}D_{\text {lum }} \\
(\mathrm{Mpc}) \\
(7)\end{array}$ & $\begin{array}{c}V_{h} \\
\left(\mathrm{~km} \mathrm{~s}^{-1}\right) \\
(8)\end{array}$ & $\begin{array}{l}\text { OBSID } \\
\text { (9) }\end{array}$ & $\begin{array}{l}\text { Exp } \\
(\mathrm{sec}) \\
(10)\end{array}$ & $\begin{array}{l}\text { FTS Program } \\
\text { (11) }\end{array}$ \\
\hline NGC 7679 & $23^{\mathrm{h}} 28^{\mathrm{m}} 46^{\mathrm{s}} \cdot 6$ & $3^{\mathrm{d}} 30^{\mathrm{m}} 43^{\mathrm{s}}$ & 1.3 & $11.11\left(^{*}\right)$ & 0.69 & 73.8 & 5138 & 1342221701 & 2936 & OT1_nlu_1 \\
\hline NGC 7771 & $23^{\mathrm{h}} 51^{\mathrm{m}} 24.7$ & $20^{\mathrm{d}} 06^{\mathrm{m}} 41^{\mathrm{s}}$ & 3.6 & $11.27\left(^{*}\right)$ & 0.49 & 61.2 & 4277 & 1342212317 & 14832 & KPOT_pvanderw_1 \\
\hline Mrk 331 & $23^{\mathrm{h}} 51^{\mathrm{m}} 26^{\mathrm{s}} \cdot 6$ & $20^{\mathrm{d}} 35^{\mathrm{m}} 09^{\mathrm{s}}$ & 3.1 & $11.50\left(^{*}\right)$ & 0.79 & 79.3 & 5541 & 1342212316 & 13616 & KPOT_pvanderw_1 \\
\hline
\end{tabular}

Notes.

${ }^{\mathrm{a}}$ A member of the subsample of powerful AGNs defined in Section 2.2.

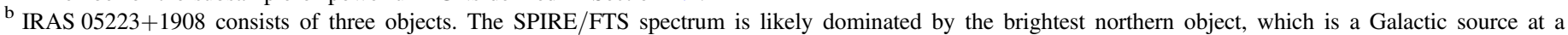

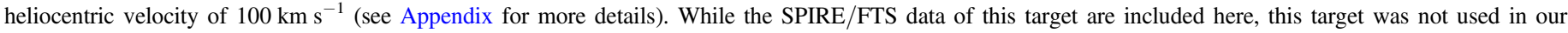
statistical analysis.

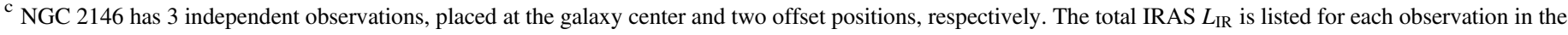
table.

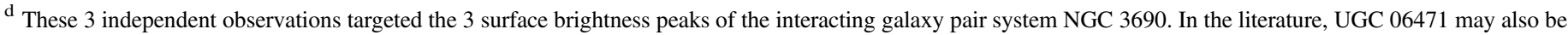

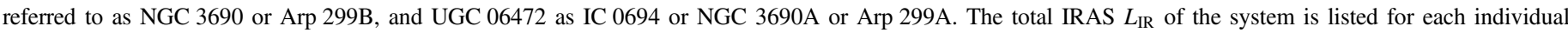
observation in the table.

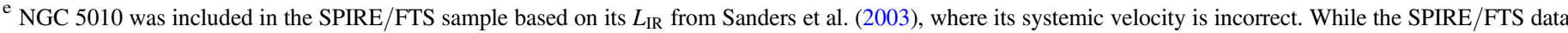
of this target are still documented here, this target was not used in any statistical analysis in this paper.

(This table is available in machine-readable form.)

Herschel-wide absolute pointing error that could be up to $2^{\prime \prime}$. Column (5) is the adopted $L_{\mathrm{IR}}$ relevant to the target of the SPIRE/FTS observation. For a target in a multiple system of 2 or more galaxies, this value is followed by a "(*)," with its derivation explained in the Appendix. In many cases, this is a 24 or $70 \mu \mathrm{m}$ flux-scaled luminosity from the IRAS total $L_{\mathrm{IR}}$ of the galaxy system. In addition, due to the updated $L_{\mathrm{IR}}$, a few of our targets (e.g., NGC 0876, NGC 2341, NGC 6285) now have $L_{\mathrm{IR}}$ slightly less than $10^{11} L_{\odot}$. These galaxies are still kept in our LIRG sample. Column (6) is the FIR color, $C(60 / 100)$. For most of the targets involved in a multiple system (see the Appendix), their $C(60 / 100)$ values are simply those for their galaxy system, which should be dominated by the brightest member galaxy, which is usually our SPIRE/FTS target. Columns (7) and (8) are respectively the luminosity distance in megaparsecs and heliocentric velocity in $\mathrm{km} \mathrm{s}^{-1}$, taken from Table 1 in Armus et al. (2009). Column (9) is the SPIRE/FTS observation identification number (OBSID). The observations with an OBSID $\leqslant 1342245858$ were done prior to OD 1110 , and thus were impacted by the SPIRE-specific 1".7 pointing offset mentioned above. ${ }^{24}$ Column (10) gives the on-target integration time of the FTS observation. Finally, column (11) identifies the original FTS program in which the observation was carried out. For example, "OT1_nlu_1" refers to our own SPIRE/FTS program.

For each target in Table 1 (except for NGC 1068), estimates of the AGN fractional contribution to the total bolometric luminosity have been recently updated by Díaz-Santos et al. (2017) using a number of independent estimators, including the line ratios $[\mathrm{Ne} \mathrm{V}] /[\mathrm{Ne}$ II] and [O IV]/[Ne II], mid-IR continuum slope, equivalent width (EW) of Polycyclic Aromatic Hydrocarbon (PAH) emission bands and the diagram of Laurent et al. (2000), following the formulation prescribed in Veilleux et al. (2009). If we denote $f_{\mathrm{AGN}}$ as the unweighted average for the fractional contribution by $\mathrm{AGN}$ to the total bolometric luminosity, from these various independent estimates, there

\footnotetext{
${ }^{24}$ Note that, since the observations of the SPIRE/FTS point-source flux calibrators in the early ODs also suffered from this 1 !" 7 systematic offset, this offset has no net effect on the point-source flux calibration.
}

are a total of 6 targets with $f_{\mathrm{AGN}}>50 \%$, meaning that there is a high likelihood, with a good consistency among the different estimators, that the AGN could be the dominant source powering the observed $L_{\mathrm{IR}}$ in these galaxies. Although the classic Seyfert galaxy NGC 1068 is not included in DíazSantos et al. (2017), alternative analyses suggest $f_{\mathrm{AGN}} \gtrsim 50 \%$ for this galaxy (Telesco \& Decher 1988; Lu et al. 2014). We therefore refer to these seven galaxies (i.e., NGC 1068, UGC 02608, NGC 1275, IRAS F05189-2524, UGC 08058 (=Mrk 231), MCG-03-34-064, and NGC 7674) as the (sub) sample of dominant AGNs in the remainder of this paper.

These seven galaxies represent the cases where the AGN clearly dominates the bolometric luminosity. While it is known that SF dominates the bolometric luminosity of most LIRGs (Petric et al. 2011; Stierwalt et al. 2013), there are certainly additional sources in our sample in which the AGN contribution to the bolometric luminosity is non-negligible according to one or more individual mid-IR diagnostics, such as the one based on the EW of the $6.2 \mu \mathrm{m}$ PAH feature (Stierwalt et al. 2013). However, in this paper we have chosen to isolate those sources where the average fractional AGN bolometric contribution is above $50 \%$, in order to identify galaxies where the AGN might be expected to have the largest impact on the molecular ISM. We refer the reader to Díaz-Santos et al. (2017) for details on individual AGN diagnostics and their relationship to the average AGN fraction value we used here.

\section{Observations, Data Reduction, and Spectral Line Results}

\subsection{SPIRE/FTS Spectroscopy}

The SPIRE/FTS (Griffin et al. 2010) uses two bolometer arrays of 37 and 19 detectors for spectral imaging in a Spectrometer Short Wavelength (SSW) coverage from 194 to $313 \mu \mathrm{m}$ and a Spectrometer Long Wavelength (SLW) coverage from 303 to $671 \mu \mathrm{m}$, respectively. The incoming light from the telescope is split into two beams. An internal moving mirror regulates the optical path difference between the two beams before they are recombined to form an interference pattern that is split again and focused onto the two detector arrays located behind their respective, broadband filters. The detectors are 
arranged in a hexagonally close-packed pattern with the spacing between them set at $\sim 33^{\prime \prime}$ for SSW and $\sim 51^{\prime \prime}$ for SLW. These are roughly equal to two beam widths. Therefore, there is always a gap of one beam width between the neighboring detectors.

Ninety-one galaxies in our complete flux-limited sample were observed in our own program (program ID: OT1_nlu_1; PI: N. Lu) with the SPIRE/FTS operating in its high-resolution (HR) staring (i.e., "sparse") mode, targeted at the nuclear position of each galaxy. The resulting spectra have a frequency-independent resolution of $1.2 \mathrm{GHz}(\delta \nu)$. This corresponds to $\lambda / \delta \lambda \sim 1218$ (or a velocity resolution of $\sim 296 \mathrm{~km} \mathrm{~s}^{-1}$ ) at the frequency (i.e., $1461.1 \mathrm{GHz}$ ) of the NII line near the blue end of the FTS frequency coverage, and to $\lambda / \delta \lambda \sim 480$ (or $\sim 750 \mathrm{~km} \mathrm{~s}^{-1}$ ) at the frequency $(576.2 \mathrm{GHz})$ of $\mathrm{CO}(5-4)$ near the long-wavelength end. For a spectrally unresolved line, the line profile is a sinc function and the effective FWHM is $1.207 \delta \nu$ or $1.44 \mathrm{GHz}$ (hereafter, referred to as $\left.\Delta \nu_{\mathrm{FWHM}}^{\mathrm{FTS}}\right)$. In addition to our own observations, a total of 31 sample galaxies were observed with SPIRE/FTS by other groups in a similar way, except for NGC 4418, which was observed in a mapping mode. The data from the central pointing of the mapping observation were used for NGC 4418. The FTS data of these targets were downloaded from the Herschel science archive. As noted in Column (11) of Table 1, these observations are mostly from the open-time key project "HerCULES" (Herschel program ID: KPOT_pvanderw_1; see Van der Werf et al. 2010; Rosenberg et al. 2015), with only a few from other programs (i.e., KPGT_cwilson01_1, see Rangwala et al. 2011; Kamenetzky et al. 2012, and Spinoglio et al. 2012; GT1_lspinogl_2, see Pereira-Santaella et al. 2013; KPGT_esturm_1, see Rosenberg et al. 2015; OT1_pogle_01_1, PI: P. Ogle).

In order to cover (i) as large a sample of LIRGs as possible for better statistics and (ii) more intrinsically faint LIRGs, our own observing program was designed to ensure detection of the mid- $J$ CO lines, e.g., $5 \leqslant J<10$, in particular, $\mathrm{CO}(6-5)$ and $\mathrm{CO}(7-6)$. The on-target integration time varied from 1332 to $7992 \mathrm{~s}$, set to detect the anticipated CO (6-5) flux at a signalto-noise ratio $(\mathrm{S} / \mathrm{N})>5$. This line flux was estimated using the M82 SPIRE/FTS spectrum (Panuzzo et al. 2010) plus an apparent correlation between the $\mathrm{CO}(3-2)$ luminosity and the FIR luminosity $\left(L_{\mathrm{FIR}}\right.$, over $\left.40-120 \mu \mathrm{m}\right)$ for LIRGs from Yao et al. (2003). As a result, our detection rate of the CO lines at $J \geqslant 10$ is relatively low. In contrast, many of the archival observations targeted the brightest local LIRGs and used longer integration times (see Table 1), and therefore have significantly better detection rates on the high-J CO lines.

\subsection{Data Reduction}

All the data, both our own and those from the Herschel archive, were homogeneously reduced using HIPE version 11, which offers a line flux accuracy on the order of $6 \%$ (Swinyard et al. 2014). (The SPIRE/FTS flux calibration accuracy for spectral lines has remained largely unchanged since HIPE version 11.) The angular extent of the majority of our targets is such that the flux is largely contained within the central detector of the SSW and/or SLW arrays, with no significant flux detected in off-axis detectors. We therefore extracted a spectrum from the central detectors based on a point-source flux calibration and used it for all our subsequent spectral line detection and analysis.
Table 2

FWHM Beam Sizes at Selected Line Frequencies

\begin{tabular}{lcccc}
\hline \hline Line & $\begin{array}{c}\text { Freq. } \\
(\mathrm{GHz})\end{array}$ & $\begin{array}{c}\text { Wavlength } \\
(\mu \mathrm{m})\end{array}$ & $\begin{array}{c}\text { FWHM } \\
(\operatorname{arcsec})\end{array}$ & Detector \\
\hline $\mathrm{CO}(4-3)$ & 461.041 & 650.7 & 42.8 & SLWC3 \\
{$[\mathrm{C} \mathrm{I}] 609 \mu \mathrm{m}$} & 492.161 & 609.1 & 38.6 & SLWC3 \\
$\mathrm{CO}(5-4)$ & 576.268 & 520.2 & 35.2 & SLWC3 \\
$\mathrm{CO}(6-5)$ & 691.473 & 433.6 & 31.2 & SLWC3 \\
$\mathrm{CO}(7-6)$ & 806.652 & 371.7 & 35.9 & SLWC3 \\
{$[\mathrm{C} \mathrm{I}] 370 \mu \mathrm{m}$} & 809.342 & 370.4 & 36.0 & SLWC3 \\
$\mathrm{CO}(8-7)$ & 921.800 & 325.2 & 40.1 & SLWC3 \\
$\mathrm{CO}(9-8)$ & 1036.912 & 289.1 & 19.0 & SSWD4 \\
$\mathrm{CO}(10-9)$ & 1151.985 & 260.4 & 17.4 & SSWD4 \\
$\mathrm{CO}(11-10)$ & 1267.014 & 236.8 & 17.3 & SSWD4 \\
$\mathrm{CO}(12-11)$ & 1381.995 & 217.1 & 16.9 & SSWD4 \\
{$[\mathrm{N} \mathrm{II}] 205 \mu \mathrm{m}$} & 1461.132 & 205.2 & 16.6 & SSWD4 \\
$\mathrm{CO}(13-12)$ & 1496.922 & 200.4 & 16.6 & SSWD4 \\
\hline
\end{tabular}

A SPIRE/FTS continuum inherits a residual signal from the bright emission of the $80 \mathrm{~K}$ telescope in an additive way. Under the HIPE 11 calibration, this residual signal is on the order of $0.5 \mathrm{Jy}$. However, this systematic effect has no impact on the detection and flux derivation of spectral lines. The SPIRE/FTS continuum fluxes are used in this paper only when we discuss the HF spectral line near the end of Section 5, where we describe how we tried to further remove this telescope residual from the continuum (see Section 5.7.2).

All of our targets have been observed with the Herschel Photodetector Array Camera and Spectrometer (PACS; Poglitsch et al. 2010) at $70 \mu \mathrm{m}$ (J. Chu et al. 2017, in preparation). Based on the PACS images, some of our targets are more extended at $70 \mu \mathrm{m}$ than the SPIRE beam relevant to the frequency of a particular spectral line under consideration. Since both the $70 \mu \mathrm{m}$ emission from warm dust (e.g., Helou 1986; Buat \& Deharveng 1988) and mid-J CO line emission in LIRGs are traced to the same star-forming regions, in such cases, the line flux from the point-source-calibrated spectrum represents a lower limit on the total line flux of the target, and an appropriate aperture flux correction is needed. Since the cold dust continuum in a SPIRE/FTS spectrum may have a different spatial scale than a mid- $J$ CO line from warm molecular gas in star-forming regions, we chose not to do the aperture flux correction for an observed $\mathrm{CO}$ line by minimizing the continuum gap between the SSW and SLW spectral segments as prescribed in $\mathrm{Wu}$ et al. (2013). Instead, we provide a line flux aperture correction factor $f_{70 \mu \mathrm{m}}^{-1}(\theta)$, where $f_{70 \mu \mathrm{m}}(\theta)$ is the fractional $70 \mu \mathrm{m}$ continuum flux within a Gaussian beam of $\theta$ (FWHM). This was done by convolving the PACS $70 \mu \mathrm{m}$ image to the Gaussian beam of $\theta$ following the convolution algorithm in Aniano et al. (2011; see Zhao et al. 2016a for more details). In Table 2, we list the SPIRE/FTS FWHM beam sizes (see the SPIRE Handbook) for all our main targeted spectral lines. We have calculated the values of $f_{70 \mu \mathrm{m}}(\theta)$ for representative beam sizes of $\theta=17^{\prime \prime}, 30^{\prime \prime}$, and $35^{\prime \prime}$ (see Table 4) for each galaxy and discuss these parameters in depth later (see Section 4.2).

\subsection{Spectra}

Figure 2 displays the final SPIRE/FTS spectra in the reference frame of local standard of rest (LSR), after the continuum was fit by a polynomial and subsequently removed 

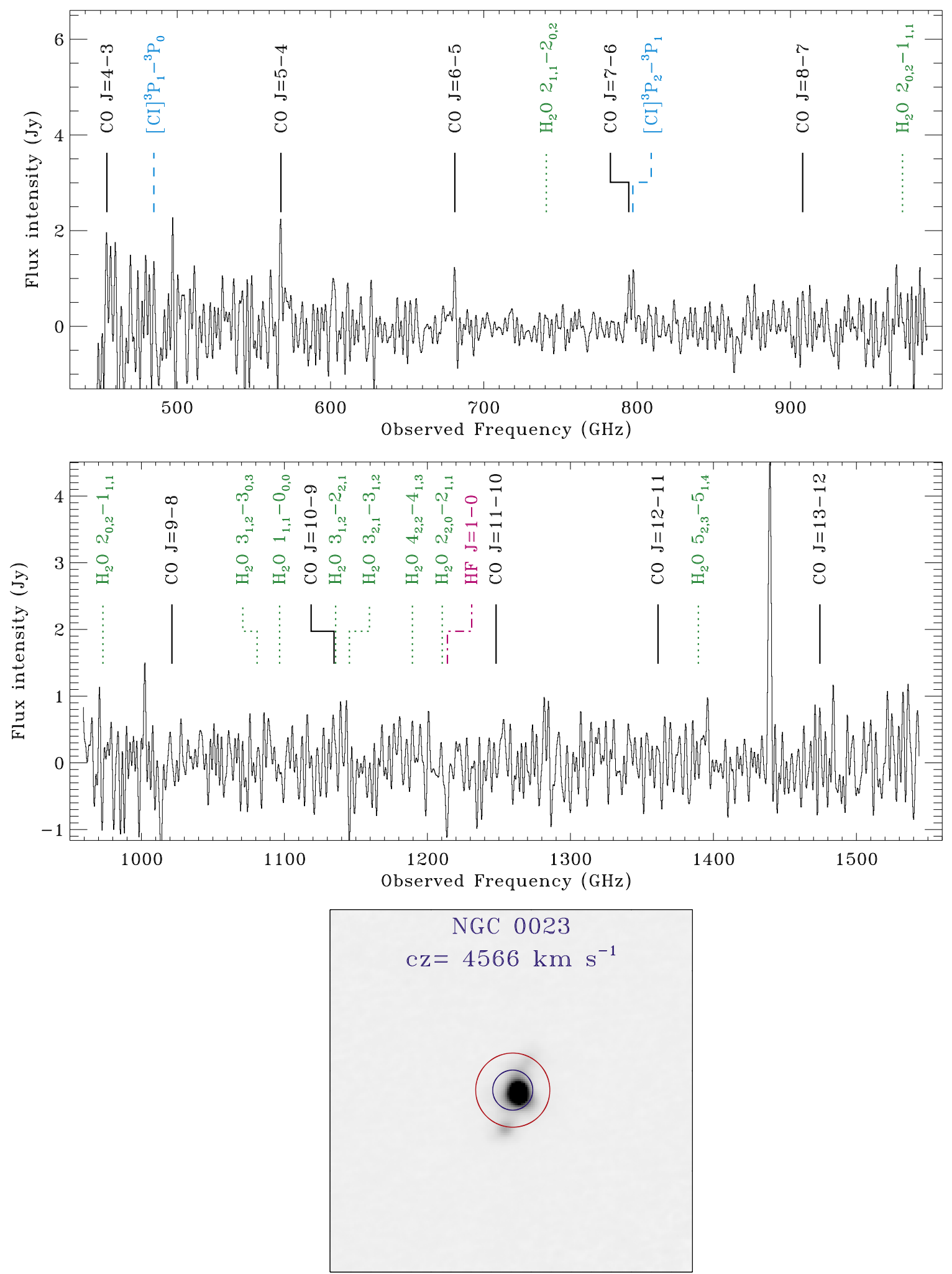

Figure 2. Continuum-subtracted spectra in the local standard of rest (LSR), with expected frequencies of the spectral lines discussed in the text marked, for the sample galaxies. The brightest feature is almost always the [N II] $205 \mu \mathrm{m}$ line, which is not marked, but can be easily identified. The grayscale image shown is the corresponding PACS $70 \mu \mathrm{m}$ image ( $3^{\prime} \times 3^{\prime}$; north up and east to the left) overlaid with the two SPIRE/FTS FWHM beam sizes at $250 \mu \mathrm{m}$ (in blue) and $500 \mu \mathrm{m}$ (red). The gray scale was set between the intensities above which $99.5 \%$ and $15 \%$ pixels lie, respectively. The target name and its heliocentric velocity are also labeled. (The complete figure set (127 images) is available.)

(as detailed below). The expected frequencies of the $\mathrm{CO}$ lines, the $[\mathrm{C} \mathrm{I}]$ lines, a few rotational transitions of $\mathrm{H}_{2} \mathrm{O}$ vapor, and $\mathrm{HF}(1-0)$ are marked and labeled in each spectral plot. The brightest line in each spectral plot is almost always [N II] $205 \mu \mathrm{m}$, and it is not marked, as it can be unambiguously recognized. For each target, the SSW spectrum is in the upper 
Table 3

Rest-frame Frequencies of Known Spectral Lines within the SPIRE/FTS Frequency Coverage

\begin{tabular}{|c|c|c|c|}
\hline Line in SLW & $\begin{array}{l}\text { Frequency } \\
\quad(\mathrm{GHz})\end{array}$ & Line in SSW & $\begin{array}{l}\text { Frequency } \\
\quad(\mathrm{GHz})\end{array}$ \\
\hline $\mathrm{CO}(4-3)$ & 461.041 & $\mathrm{OH}^{+}\left(1_{12}-0_{12}\right)^{\mathrm{a}}$ & 1033.118 \\
\hline$[\mathrm{C} \mathrm{I}](1-0)$ & 492.161 & $\mathrm{CO}(9-8)^{\mathrm{a}}$ & 1036.912 \\
\hline $\mathrm{HCN}(6-5)$ & 531.716 & $\mathrm{HCN}(12-11)$ & 1062.980 \\
\hline $\mathrm{HCO}^{+}(6-5)$ & 536.828 & $\mathrm{H}_{2} \mathrm{O}\left(3_{12}-3_{03}\right)$ & 1097.365 \\
\hline${ }^{13} \mathrm{CO}(5-4)$ & 550.926 & $\mathrm{H}_{2} \mathrm{O}\left(1_{11}-0_{00}\right)$ & 1113.343 \\
\hline $\mathrm{CO}(5-4)$ & 576.268 & $\begin{array}{l}\mathrm{H}_{2} \mathrm{O} \\
\quad+\left(1_{11}-0_{00}\right. \\
\left.\quad J_{3 / 2-1 / 2}\right)\end{array}$ & 1115.204 \\
\hline $\mathrm{HCO}^{+}(7-6)$ & 624.208 & $\begin{array}{l}\mathrm{H}_{2} \mathrm{O} \\
\quad+\left(1_{11}-0_{00}\right. \\
\left.\quad J_{1 / 2-1 / 2}\right)\end{array}$ & 1139.561 \\
\hline${ }^{13} \mathrm{CO}(6-5)$ & 661.067 & $\mathrm{CO}(10-9)$ & 1151.985 \\
\hline $\mathrm{CO}(6-5)$ & 691.473 & $\mathrm{H}_{2} \mathrm{O}\left(3_{12}-2_{21}\right)$ & 1153.127 \\
\hline $\begin{array}{c}\mathrm{H}_{2} \mathrm{O}^{+}\left(2_{02}-1_{11}\right. \\
\left.J_{5 / 3-3 / 2}\right)\end{array}$ & 742.033 & $\mathrm{H}_{2} \mathrm{O}\left(3_{21}-3_{12}\right)$ & 1162.912 \\
\hline $\begin{array}{c}\mathrm{H}_{2} \mathrm{O}^{+}\left(2_{02}-1_{11}\right. \\
\left.J_{3 / 2-3 / 2}\right)\end{array}$ & 746.194 & $\mathrm{H}_{2} \mathrm{O}\left(4_{22}-4_{13}\right)$ & 1207.639 \\
\hline $\mathrm{H}_{2} \mathrm{O}\left(2_{11}-2_{02}\right)$ & 752.033 & $\mathrm{H}_{2} \mathrm{O}\left(2_{20}-2_{11}\right)$ & 1228.789 \\
\hline${ }^{13} \mathrm{CO}(7-6)$ & 771.184 & $\mathrm{HF}(1-0)$ & 1232.476 \\
\hline $\mathrm{CO}(7-6)$ & 806.652 & $\operatorname{HCN}(14-13)$ & 1239.890 \\
\hline$[\mathrm{C} \mathrm{I}](2-1)$ & 809.342 & $\mathrm{CO}(11-10)$ & 1267.014 \\
\hline $\mathrm{CH}^{+}(1-0)$ & 835.079 & $\operatorname{HCN}(15-14)$ & 1328.302 \\
\hline${ }^{13} \mathrm{CO}(8-7)$ & 881.273 & $\mathrm{CO}(12-11)$ & 1381.995 \\
\hline $\mathrm{OH}^{+}\left(1_{01}-0_{12}\right)$ & 909.159 & $\mathrm{H}_{2} \mathrm{O}\left(5_{23}-5_{14}\right)$ & 1410.618 \\
\hline $\mathrm{CO}(8-7)$ & 921.800 & $\operatorname{HCN}(16-15)$ & 1416.683 \\
\hline $\mathrm{OH}^{+}\left(1_{22}-0_{11}\right)^{\mathrm{b}}$ & 971.805 & {$[\mathrm{~N}$ II $](1-0)$} & 1461.134 \\
\hline \multirow[t]{3}{*}{$\mathrm{H}_{2} \mathrm{O}\left(2_{02}-1_{11}\right)^{\mathrm{b}}$} & 987.927 & $\mathrm{CO}(13-12)$ & 1496.923 \\
\hline & & $\operatorname{HCN}(17-16)$ & 1505.030 \\
\hline & & $\mathrm{CO}(14-13)$ & 1611.793 \\
\hline
\end{tabular}

Notes.

${ }^{a}$ These lines could also be seen in the SLW array if the source heliocentric velocity is high enough, i.e., greater than about 13,510 and $14,660 \mathrm{~km} \mathrm{~s}^{-1}$ for $\mathrm{OH}^{+}\left(1_{12}-0_{12}\right)$ and $\mathrm{CO}(9-8)$, respectively.

$\mathrm{b}$ These lines could also be seen in the SSW array if the source heliocentric velocity is low enough, i.e., less than about 4640 and $9695 \mathrm{~km} \mathrm{~s}^{-1}$ for $\mathrm{OH}^{+}\left(1_{22}-0_{11}\right)$ and $\mathrm{H}_{2} \mathrm{O}\left(2_{02}-1_{11}\right)$, respectively.

panel, followed by the SLW spectrum and then by the corresponding PACS $70 \mu \mathrm{m}$ image of $3^{\prime} \times 3^{\prime}$ in size, overlaid with the two SPIRE/FTS FWHM beam sizes at $250 \mu \mathrm{m}$ and $500 \mu \mathrm{m}$, respectively. This PACS image should be used only as a quick visual guide as to whether the target is significantly extended with respect to the FTS beams. We refer the reader to J. Chu et al. (2017, in preparation) for a more detailed analysis of the PACS images.

The SPIRE/FTS spectrum of IRAS $05223+1908$ is dominated by a set of strong $\mathrm{CO}$ lines, which can be best fit with a heliocentric velocity of $100 \mathrm{~km} \mathrm{~s}^{-1}$. A close inspection of the PACS images of this target reveals three distinct objects. As explained in Appendix, the spectral lines in the SPIRE/FTS spectrum are dominated by the emission from the northern object in the field, which is likely a Galactic source. The data for this target, as well as for the non-LIRG galaxy NGC 5010 we observed, are included in this paper (i.e., in Tables 1, 3 and 5) for completeness, however not used in any analyses.

For targets close to the Galactic plane, the Galactic [N II] $205 \mu \mathrm{m}$ line emission could be present in their spectra, usually at a frequency close to CO (13-12). ESO 099-G004 and IRAS 08355-4944 in Figure 2 are two such examples.

\subsection{Line Detection, Flux Derivation, and Identification}

Tabulated in Table 3 is a list of the spectral lines (in emission or absorption) detected in one of the deep SPIRE/FTS spectra of Arp 220 (a ULIRG; Rangwala et al. 2011), M82 (a starburst; Kamenetzky et al. 2012), and NGC 1068 (an AGN; Spinoglio et al. 2012). We then searched for these lines in each of our sample galaxies. We opted for this approach, rather than a blind search for lines, because of the consideration that many of our spectra are sensitive enough to detect only our primary targeted lines. This approach minimizes the number of spurious line detections. On the other hand, a potential drawback from this approach is that we might miss spectral lines that are not in Table 3, but would have been detected purely based on a $\mathrm{S} / \mathrm{N}$ criterion. In practice, this is only a potential issue in the cases of the highest-S/N spectra obtained in the HerCULES program (see Table 1): indeed, a few spectral lines additional to those compiled in Table 3 have been possibly detected in some HerCULES spectra (P. Van der Werf 2016, private communication).

The determination of the continuum emission is a two-step process. First, we fit a polynomial (of order 5) to the observed SSW or SLW spectrum. A global channel-to-channel rms noise was calculated after subtracting the polynomial fit from the spectrum. Then all the spectral features (either in emission or absorption) with a peak signal-to-noise ratio greater than 3 were identified and further masked out using a box car of 10 (3.0 GHz; for SLW) or 14 (4.2 GHz; for SSW) sample points. A new polynomial fit of the order of 5 was then applied to all the remaining data samples, resulting in our final continuum fit.

The detection of a candidate spectral line in Table 3 was done in two steps. First, we calculated an average noise, $\sigma_{\text {local }}^{\mathrm{r}}$, from the noise spectrum provided as part of the SPIRE/FTS pipeline product, but within a spectral window of 20 times $\Delta \nu_{\mathrm{FWHM}}^{\mathrm{FTS}}$, centered at the redshifted frequency of the spectral line under consideration. The line was deemed as a tentative detection if there was a signal peak within $\pm \Delta \nu_{\mathrm{FWHM}}^{\mathrm{FTS}}$ of the expected line frequency, and with an amplitude of $>2.5 \times \sigma_{\text {local }}^{\mathrm{r}}$. The quantity $\sigma_{\text {local }}^{\mathrm{r}}$ mainly reflects the random noise, and is usually equal to or somewhat smaller than the total noise in the spectrum. The latter includes systematic noise due to imperfect FTS calibration. Therefore this tentative detection criterion is more relaxed than a true $\mathrm{S} / \mathrm{N}=2.5$ criterion. Once all the tentatively detected lines were identified, these lines were fit simultaneously. For each feature, we used either a sinc or a sinc-Gaussian convolved line profile, plus a local linear function for any possible residual continuum limited to the data points within a frequency window of $20 \Delta \nu_{\mathrm{FWHM}}^{\mathrm{FTS}}$ in width, centered at the frequency of the feature peak. The sinc function in either case always had a fixed width (in frequency) equal to that of the SPIRE/FTS instrumental resolution profile. The Gaussian component of the sinc-Gaussian function had a free width parameter to reflect the unknown velocity dispersion of a spectral line. The collective model fit to all the tentatively detected features was obtained using an interactive data language nonlinear least-squares fitting procedure as prescribed by Markwardt (2009) and was subsequently removed from the target spectrum. The resulting "line-free" residual spectrum was used to calculate a refined local (total) noise, $\sigma_{\text {local }}^{t}$, for each tentatively detected spectral line. This noise was the rms 
value (after further removing the fitted local residual continuum) within a frequency window of $20 \Delta \nu_{\mathrm{FWHM}}^{\mathrm{FTS}}$ in length, centered on the fitted central frequency of the spectral line under consideration. Finally, all tentative detected lines with a fitted peak flux density greater than $3 \sigma_{\text {local }}^{t}$ were retained as being the formal line detections. In the remainder of this paper, the $\mathrm{S} / \mathrm{N}$ of a line always refers to the ratio of the line peak signal to $\sigma_{\text {local }}^{t}$.

In principle, the sinc profile always underestimates the flux of a line because the line always has some intrinsic width (see Section 4 for more details on this). However, a sinc-Gaussian line profile is usually less robust than that using a sinc-only profile, as the former line template is more sensitive to the wings of a spectral line. As a result, one usually requires a very high $\mathrm{S} / \mathrm{N}$ in order to obtain a reliable line fit using a sincGaussian profile if the line is unresolved or only marginally resolved, which should be the case for the mid- $J$ CO lines in all our galaxies (but NGC 6240). As a result, we used a sinconly line profile for all the detected lines (except for the [N II] line), with the frequency width of the sinc function set to the SPIRE/FTS spectral resolution. (The resulting line flux could underestimate the real flux if the line significantly resolved. We address this potential systematic effect in more detail in Section 4.) For the [N II] line, we always tried to fit a sincGaussian profile if possible. This choice is not only practical because the $[\mathrm{N}$ II] line was usually detected at a high $\mathrm{S} / \mathrm{N}$, but also logical since the line is near the blue end of the SPIRE/ FTS spectral coverage. In a few cases, we used the sinc-only profile to fit the [N II] line, as this resulted in a better fit. For NGC 6240, its CO and [C I] lines are quite broad and have high $\mathrm{S} / \mathrm{Ns}$ (see Table 4). We fit each of these lines with a sincGaussian profile as well.

For each detected line, we assign a quality flag $Q$. This flag depends on the $\mathrm{S} / \mathrm{N}$ and a velocity criterion that measures how well the heliocentric velocity $\left(V_{\text {obs }}\right)$ inferred from the fitted line central frequency matches a fiducial velocity $\left(V_{\text {fiducial }}\right)$ adopted for the target. Figure 3 shows the observed rms value of the velocity difference, $\left(V_{\mathrm{obs}}-V_{[\mathrm{N} \mathrm{II}]}\right)$, for all those lines that have been detected at $\mathrm{S} / \mathrm{N} \geqslant 7$ in at least 3 targets, where $V_{[\mathrm{N} \mathrm{II}}$ is the inferred heliocentric velocity from the fitted central line frequency of the $[\mathrm{N} \mathrm{II}]$ line. For a given spectral line, this rms value was calculated over the number of qualifying targets. With such a high $\mathrm{S} / \mathrm{N}$ cutoff, we were looking for any potential systematic trend of these rms velocity differences as a function of frequency, for example, as a result of decreasing velocity resolution with decreasing frequency; no obvious trend is seen in Figure 3. The maximum value of this rms velocity difference across all the lines shown in Figure 3 is $\sigma_{V} \sim 70 \mathrm{~km} \mathrm{~s}^{-1}$. We therefore assigned a good quality flag to a detected line if $\left|V_{\text {obs }}-V_{\text {fiducial }}\right|<210 \mathrm{~km} \mathrm{~s}^{-1}$ (i.e., $3 \sigma_{V}$ ), where $V_{\text {fiducial }}$ was set to the inferred velocity of the $[\mathrm{N}$ II] line. In a rare case in which the [N II] line was not detected, $V_{\text {fiducial }}$ was set to the averaged velocity of the detected CO lines. Additional details on the $Q$ flag are given in Table 4 . We found no cases in which one particular suite of lines, e.g., $\mathrm{CO}$, have consistent velocities among themselves, but differ in velocity from that of the [N II] line at a significance of $3 \sigma_{V}$ or larger.

Figure 4 shows examples of CGCG 049-057 and NGC 6240. The sinc line profiles were used to fit all lines but (i) the [N II] $205 \mu \mathrm{m}$ line in the spectra of both galaxies and (ii) the $\mathrm{CO}$ and [C I] lines in the spectrum of NGC 6240. The lines specified in (i) and (ii) were fit with sinc-Gaussian profiles.

\subsection{Results}

Table 4 tabulates the derived line fluxes and other properties of the CO rotational transitions of $J=4-13$, the two [C I] lines and the $[\mathrm{N}$ II] line. The table columns are as follows.

Column (1) is the target name from Table 1.

Columns (2)-(14) contain the data for each of these spectral lines, in 9 rows, where:

Row 1-The spectral line flux or upper limit in units of $10^{-17} \mathrm{~W} \mathrm{~m}^{-2}$. A positive number indicates that the line is detected, while a negative number represents a non-detection with its absolute value being the $3 \sigma$ upper limit, where $\sigma$ was set using a sinc line profile together with the local rms noise around the expected line frequency. For $\mathrm{CO}(4-3)$, a flux value of zero means that the line is redshifted out of the low frequency end of the SLW coverage. This is the case for a total of 17 targets with $V_{h} \leqslant 9558 \mathrm{~km} \mathrm{~s}^{-1}$. Note that the flux values given here (as well as in Table 5) were derived assuming a point-source case. See Section 4.2 for a discussion on how to use the $f_{70 \mu \mathrm{m}}(\theta)$ factors in Column (15) of Table 4 for a possible flux aperture correction if the target is more extended than a point-source. Row entries 2 to 9 are relevant only if the line is detected.

Row 2-The line flux uncertainty in units of $10^{-17} \mathrm{~W} \mathrm{~m}^{-2}$. This is the uncertainty from the line-fitting procedure. For most of the spectral lines fitted with a sinc profile, this was found to be comparable to $F /(\mathrm{S} / \mathrm{N})$, where $F$ is the total line flux in Row 1 and $\mathrm{S} / \mathrm{N}$ is the ratio of the line peak to $\sigma_{\text {local }}^{t}$, given in Row 7 in this table. This local noise $\sigma_{\text {local }}^{t}$-based estimator tends to overestimate the real line flux uncertainty because the line flux fitting was done over multiple data points. Near the long-wavelength ends of both SLW and SSW, the spectral noise appears to be more "spiky" than Gaussian noise due to some systematic noise. Therefore $\sigma_{\text {local }}^{t}$ could be systematically larger than the line flux uncertainty quoted in Row 2 here. The spectral lines that are susceptible to this potential issue include $\mathrm{CO}(4-3)$, [C I] $609 \mu \mathrm{m}$, and possibly $\mathrm{CO}(9-8)$.

Row 3-The observed central frequency of the line, in $\mathrm{GHz}$, in the LSR.

Row 4-The uncertainty of the observed line central frequency, in $\mathrm{GHz}$, from the line profile fit.

Row 5-The difference in $\mathrm{GHz}$ between the observed line central frequency and the expected line frequency based on the heliocentric velocity of the target in Table 1 .

Row 6-The peak line flux density in Jy.

Row 7-The $\mathrm{S} / \mathrm{N}$ of the peak line flux density to the local rms noise $\sigma_{\text {local }}^{t}$.

Row 8-A quality flag, $Q$, assigned for a detected line, with $Q=1$ : a robust detection with a $\mathrm{S} / \mathrm{N} \geqslant 5$ and a satisfaction of our velocity criterion of $\left|V_{\text {obs }}-V_{\text {fiducial }}\right|<210 \mathrm{~km} \mathrm{~s}^{-1}$ (as defined in Section 3.4 above); $Q=2$ : a less robust detection with $3 \leqslant \mathrm{~S} / \mathrm{N}<5$ but still satisfying our velocity criterion; $Q=3$ : a good detection with $\mathrm{S} / \mathrm{N}>5$, but a possible line identification with the inferred line velocity being just short of satisfying our velocity criterion; or $Q=4$ : a detection of $3 \leqslant \mathrm{~S} / \mathrm{N}<5$ and only a possible line identification with the inferred line velocity being just short of satisfying our velocity criterion.

Row 9-The FWHM of the Gaussian component in $\mathrm{km} \mathrm{s}^{-1}$ when a sinc-Gaussian profile was used for the line-fitting. 
Table 4

Fluxes of the CO, [C I] and [N II] Lines

\begin{tabular}{|c|c|c|c|c|c|c|c|c|c|c|c|c|c|c|}
\hline $\begin{array}{l}\text { Name } \\
\text { (1) }\end{array}$ & $\begin{array}{c}(4-3) \\
(2)\end{array}$ & $\begin{array}{c}(5-4) \\
(3)\end{array}$ & $\begin{array}{c}(6-5) \\
(4)\end{array}$ & $\begin{array}{c}(7-6) \\
(5)\end{array}$ & $\begin{array}{c}(8-7) \\
(6)\end{array}$ & $\begin{array}{c}(9-8) \\
(7)\end{array}$ & $\begin{array}{c}(10-9) \\
(8)\end{array}$ & $\begin{array}{c}(11-10) \\
(9)\end{array}$ & $\begin{array}{c}(12-11) \\
(10)\end{array}$ & $\begin{array}{c}(13-12) \\
(11)\end{array}$ & $\begin{array}{c}{[\mathrm{C} \text { I }] 609} \\
(12)\end{array}$ & $\begin{array}{c}{[\mathrm{C} \text { I] } 370} \\
(13)\end{array}$ & $\begin{array}{c}{[\mathrm{N} \text { II] }} \\
(14)\end{array}$ & $\begin{array}{c}f_{70 \mu \mathrm{m}}(\theta) \\
(15)\end{array}$ \\
\hline \multirow[t]{9}{*}{ NGC 0023} & -4.22 & 2.57 & 1.43 & 1.14 & -1.34 & -1.14 & -1.31 & -1.35 & -1.22 & -1.33 & -2.98 & 1.32 & 10.94 & 0.95 \\
\hline & $\ldots$ & 0.32 & 0.13 & 0.12 & $\ldots$ & $\ldots$ & $\ldots$ & $\ldots$ & $\ldots$ & $\ldots$ & $\ldots$ & 0.11 & 0.66 & 0.92 \\
\hline & $\ldots$ & 567.39 & 681.02 & 794.86 & $\ldots$ & $\ldots$ & $\ldots$ & $\ldots$ & $\ldots$ & $\ldots$ & $\ldots$ & 797.44 & 1439.42 & 0.75 \\
\hline & $\ldots$ & 0.09 & 0.06 & 0.09 & $\ldots$ & $\ldots$ & $\ldots$ & $\ldots$ & $\ldots$ & $\ldots$ & $\ldots$ & 0.06 & 0.04 & $\ldots$ \\
\hline & $\ldots$ & -0.23 & -0.08 & 0.31 & $\ldots$ & $\ldots$ & $\ldots$ & $\ldots$ & $\ldots$ & $\ldots$ & $\ldots$ & 0.24 & 0.20 & $\ldots$ \\
\hline & $\ldots$ & 2.17 & 1.20 & 0.96 & $\ldots$ & $\ldots$ & $\ldots$ & $\ldots$ & $\ldots$ & $\ldots$ & $\ldots$ & 1.12 & 5.00 & $\ldots$ \\
\hline & $\ldots$ & 4.43 & 4.62 & 4.80 & $\ldots$ & $\ldots$ & $\ldots$ & $\ldots$ & $\ldots$ & $\cdots$ & $\ldots$ & 5.33 & 18.52 & $\ldots$ \\
\hline & $\ldots$ & 2 & 2 & 2 & $\ldots$ & $\ldots$ & $\ldots$ & $\ldots$ & $\ldots$ & $\ldots$ & $\ldots$ & 1 & 1 & $\ldots$ \\
\hline & $\ldots$ & $\ldots$ & $\ldots$ & $\ldots$ & $\ldots$ & $\ldots$ & $\ldots$ & $\ldots$ & $\ldots$ & $\ldots$ & $\ldots$ & $\ldots$ & 428.10 & $\ldots$ \\
\hline \multirow[t]{9}{*}{ NGC 0034} & -0.83 & 1.54 & 1.91 & 2.11 & 2.05 & 2.20 & 1.66 & 1.66 & 1.17 & 0.93 & 0.88 & 0.97 & 3.52 & 1.00 \\
\hline & $\ldots$ & 0.11 & 0.06 & 0.05 & 0.09 & 0.09 & 0.08 & 0.08 & 0.07 & 0.08 & 0.13 & 0.05 & 0.23 & 0.98 \\
\hline & $\ldots$ & 565.28 & 678.33 & 791.24 & 904.36 & 1017.21 & 1130.22 & 1242.87 & 1355.98 & 1468.29 & 482.98 & 794.20 & 1433.43 & 0.85 \\
\hline & $\ldots$ & 0.04 & 0.02 & 0.02 & 0.03 & 0.03 & 0.03 & 0.03 & 0.04 & 0.06 & 0.10 & 0.03 & 0.03 & $\ldots$ \\
\hline & $\ldots$ & 0.10 & 0.17 & 0.11 & 0.29 & 0.25 & 0.40 & 0.24 & 0.57 & 0.17 & 0.29 & 0.43 & 0.40 & $\ldots$ \\
\hline & $\ldots$ & 1.30 & 1.61 & 1.78 & 1.73 & 1.85 & 1.40 & 1.40 & 0.99 & 0.79 & 0.74 & 0.82 & 1.88 & $\ldots$ \\
\hline & $\ldots$ & 10.83 & 14.64 & 22.25 & 11.53 & 13.21 & 8.24 & 12.73 & 7.07 & 4.65 & 5.69 & 11.71 & 15.67 & $\ldots$ \\
\hline & $\ldots$ & 1 & 1 & 1 & 1 & 1 & 1 & 1 & 1 & 2 & 1 & 1 & 1 & $\ldots$ \\
\hline & $\ldots$ & $\ldots$ & $\ldots$ & $\ldots$ & $\ldots$ & $\ldots$ & $\ldots$ & $\ldots$ & $\ldots$ & $\ldots$ & $\ldots$ & $\ldots$ & 368.10 & \\
\hline
\end{tabular}

\section{Notes.}

${ }^{\mathrm{a}}$ The spectrum of IRAS $05223+1908$ shows strong CO lines that have an inferred heliocentric velocity of $100 \mathrm{~km} \mathrm{~s}^{-1}$. This velocity was used for other line detections.

b These $f_{70 \mu \mathrm{m}}(\theta)$ values are all with respect to the total $70 \mu \mathrm{m}$ flux of the galaxy NGC 2146 .

${ }^{\mathrm{c}}$ These $f_{70 \mu \mathrm{m}}(\theta)$ values are all with respect to the integrated $70 \mu \mathrm{m}$ flux of the whole NGC 3690 system.

(This table is available in its entirety in machine-readable form.) 


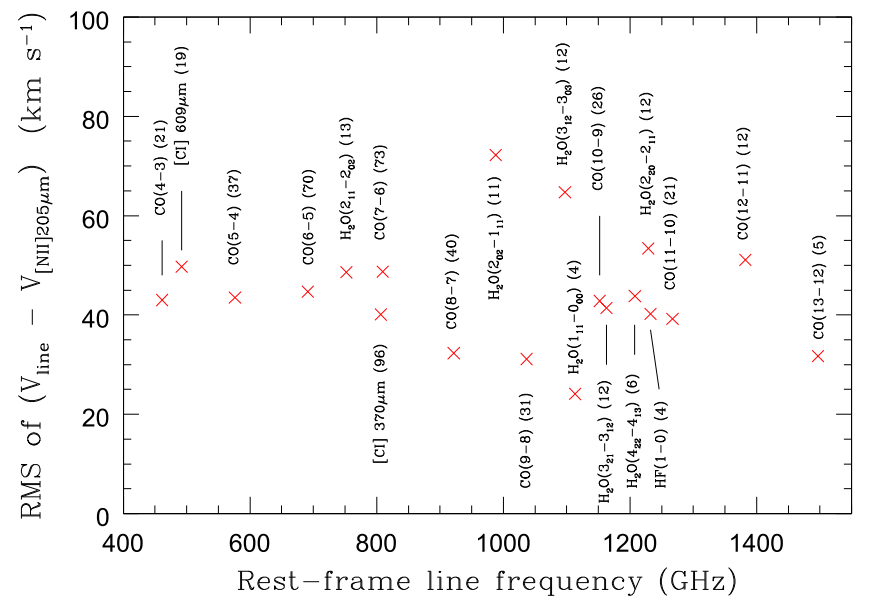

Figure 3. Plot of the rms value of $\left(V_{\text {line }}-V_{[\mathrm{N} \mathrm{II}]}\right)$ of a spectral line as a function of the line frequency, where $V_{\text {line }}$ is the inferred heliocentric velocity of the spectral line and $V_{[\mathrm{N} \mathrm{II}}$ is the similar velocity of the $[\mathrm{N} \mathrm{II}]$ line. The rms value was calculated using all the targets in which the line was detected at $\mathrm{S} / \mathrm{N} \geqslant 7$. Only the spectral lines that were detected at $S / N \geqslant 7$ for at least three targets are plotted here. For each spectral line, the number of targets used in the calculation of this rms value is given in parentheses next to the label of the line.

Column (15) gives the data of $f_{70 \mu \mathrm{m}}(\theta)$ for three different values of $\theta$ :

Row $1-f_{70 \mu \mathrm{m}}\left(35^{\prime \prime}\right)$, appropriate for the SPIRE/FTS beam sizes of the $\mathrm{CO}(5-4), \mathrm{CO}(7-6)$ or [C I] 370 lines.

Row $2-f_{70 \mu \mathrm{m}}\left(30^{\prime \prime}\right)$, appropriate for the SPIRE/FTS beam size of the $\mathrm{CO}(6-5)$ line.

Row $3-f_{70 \mu \mathrm{m}}\left(17^{\prime \prime}\right)$, appropriate for the SPIRE/FTS beam size of the [N II] line and the higher-J CO lines covered by the SSW spectral segment.

Table 5 lists those targets with one or more additional lines detected. These lines, which are listed in Column (2) of the table, are usually fainter than our main targeted lines in Table 4 and include, for example, a set of rotational transitions of $\mathrm{H}_{2} \mathrm{O}$ vapor and $\mathrm{HF}(1-0)$.

With the frequency coverage of SSW starting at $\sim 957 \mathrm{GHz}$ and that of SLW ending at $\sim 989 \mathrm{GHz}$, the two arrays have a frequency coverage overlap of $\sim 32 \mathrm{GHz}$. As noted in Table 3, up to 2 of the following targeted spectral lines could be seen in both arrays, depending on the source heliocentric velocity: $\mathrm{CO}(9-8)$ and $\mathrm{OH}^{+}\left(1_{12}-0_{12}\right)$ if $V_{h}$ is greater than 14,660 and $13,510 \mathrm{~km} \mathrm{~s}^{-1}$, respectively; $\mathrm{OH}^{+}\left(1_{22}-0_{11}\right)$ and $\mathrm{H}_{2} \mathrm{O}\left(2_{02}-1_{11}\right)$ if $V_{h}$ is less than 4640 and $9695 \mathrm{~km} \mathrm{~s}^{-1}$, respectively. For each of these lines, the line detection was performed on each detector array independently. If the line was detected in both SSW and SLW, the detection of the higher $\mathrm{S} / \mathrm{N}$ was chosen in the end. The fluxes of the $\mathrm{CO}(9-8)$ and $\mathrm{OH}^{+}\left(1_{12}-0_{12}\right)$ lines were all measured in the SSW array. For the other two lines, their fluxes given in Table 5 end with suffix "L" (for SLW) or "S" (SSW) to indicate from which detector array the flux was taken.

\section{Consideration of Systematic Effects}

\subsection{Partially Resolved Lines}

Some of the CO lines, especially those in the SSW spectral segment, may be partially resolved by the SPIRE/FTS instrumental spectral resolution. As a result, a sinc profilebased line flux derivation may underestimate the true line flux.
In many cases, these lines are either undetected or detected at a modest $\mathrm{S} / \mathrm{N}$, precluding an accurate line fit using a sincGaussian profile.

Figure 5 plots the theoretical prediction of the ratio of the line flux of a sinc-Gaussian line profile to the flux of a sinc profile with the same peak flux density, as a function of the line frequency for a number of velocity widths (FWHM) of the Gaussian component. In both cases, the width of the sinc function was fixed in frequency to correspond to that of the SPIRE/FTS instrumental resolution. It is clear that, near the blue end of the SSW spectral coverage, a line velocity dispersion of $200-300 \mathrm{~km} \mathrm{~s}^{-1}$ (in FWHM) could result in a significant flux underestimate if the sinc-only profile is used to derive the line flux. On the other hand, Figure 5 shows that, for $\mathrm{CO}(7-6)$, the line flux from the sinc profile fitting may underestimate the line flux by less than $20 \%$ if the intrinsic line FWHM is under $400 \mathrm{~km} \mathrm{~s}^{-1}$.

The majority of the archival spectra have quite high $\mathrm{S} / \mathrm{Ns}$ for $\mathrm{CO}(6-5), \mathrm{CO}(7-6),[\mathrm{CI}] 370 \mu \mathrm{m}$, and the [N II] lines. We have fit both sinc-only and sinc-Gaussian profiles to these lines in 31 archival spectra, of which the average $\mathrm{S} / \mathrm{Ns}$ are 34,30 , 26, and 45 for these four spectral lines, respectively. The resulting flux ratios are shown in Figure 6 as a function of the fitted Gaussian FWHM expressed in $\mathrm{GHz}$, where the dotted curve is a third-order polynomial representation of the theoretical prediction from Figure 5, given in Equation (1):

$$
R_{\text {theoretical }}=1.013-0.074 W+0.301 W^{2}-0.0307 W^{3} \text {, }
$$

and the solid curve is a third-order polynomial fit to the data points in Figure 15, given in Equation (2):

$$
R_{\mathrm{fit}}=1.051-0.110 W+0.315 W^{2}-0.0283 W^{3} .
$$

In both equations, $W$ is the FWHM (in $\mathrm{GHz}$ ) of the Gaussian component and $R$ stands for the (sinc-Gaussian to sinc) line flux ratio. The rms residual (along the $Y$-axis) between the data points and the solid curve is 0.03 . This plot shows that, for example, for an intrinsic line with of $1.0 \mathrm{GHz}$ (equivalent to $\sim 434$ and $372 \mathrm{~km} \mathrm{~s}^{-1}$ for $\mathrm{CO}(6-5)$ and $\mathrm{CO}(7-6)$, respectively), the sinc-only profile-based fluxes may underestimate the real flux by $\sim 20 \%$ for both of these lines.

\subsection{Flux Aperture Corrections}

The SPIRE/FTS beam depends on frequency in a non-trivial way (Swinyard et al. 2014). Table 2 lists the Gaussian FWHM beam sizes at the rest-frame frequencies of all our main targeted lines (see the SPIRE Handbook). This Gaussian approximation of the SPIRE/FTS beam should be adequate for any target that is either a true point-source or is only modestly extended with respect to the SPIRE/FTS beam. All our targets fall under one of these cases. For lines covered in the SLW spectral segment, the smallest FWHM beam size is $\sim 30^{\prime \prime}$ (i.e., for CO $(6-5)$ ). For $\mathrm{CO}(7-6)$, the relevant beam size is $\sim 35^{\prime \prime}$. For lines covered by the SSW part, a representative FWHM beam size is $\sim 17^{\prime \prime}$, as the beam size only weakly depends on frequency. For each target, we therefore list in Table 4 the values of $f_{70 \mu \mathrm{m}}(\theta)$ for $\theta=35^{\prime \prime}, 30^{\prime \prime}$, and $17^{\prime \prime}$, respectively.

Figure 7 shows how $f_{70 \mu \mathrm{m}}(\theta)$ varies with either $C(60 / 100)$ or $L_{\mathrm{IR}}$ for $\theta=30^{\prime \prime}$ (top panel) and $17^{\prime \prime}$ (bottom panel). While the former $\theta$ value is a conservative choice for all the spectral lines in the SLW spectral segment, the latter case is appropriate for the [N II] line and other lines in the SSW segment. The 


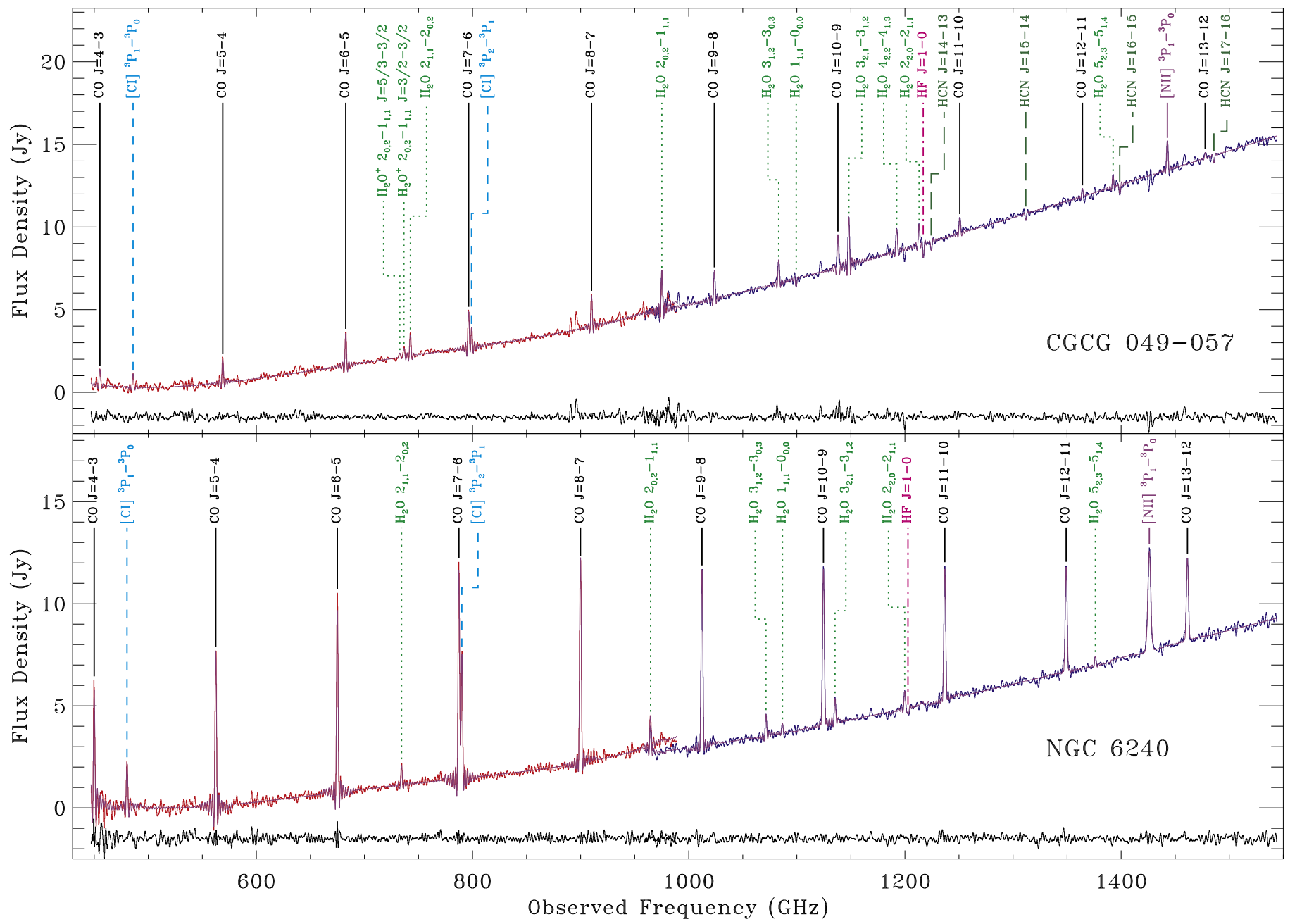

Figure 4. Examples of our line-fitting results for CGCG 049-057 (top) and NGC 6240 (bottom). In each case, we show the observed spectrum (in red or blue), overlaid with the fitted model continuum and line profiles (black), as well as their difference spectrum (also in black). The [N II] lines in both cases were fit using a sinc-Gaussian profile. All the other lines were fit using sinc-only profiles, except for the CO and [C I] lines in the case of NGC 6240, for which sinc-Gaussian profiles were used.

dotted lines in both plots indicate $f_{70 \mu \mathrm{m}}(\theta)=0.8$. For the FIR coldest (i.e., $C(60 / 100) \leqslant 0.6)$ or least luminous $\left(L_{\mathrm{IR}}\right.$ $<10^{11.3} L_{\odot}$ ) sample galaxies, about $85 \%$ and $50 \%$ of them pass the criteria $f_{70 \mu \mathrm{m}}\left(30^{\prime \prime}\right) \geqslant 0.8$ and $f_{70 \mu \mathrm{m}}\left(17^{\prime \prime}\right) \geqslant 0.8$, respectively. When considering the ratio of a SPIRE/FTS spectral line flux from this paper to the total IR or FIR flux, one could either (a) divide $f_{70 \mu \mathrm{m}}(\theta)$ into the line flux in this paper as an effective aperture correction or (b) multiply the total IR or FIR flux by this factor to arrive at an estimate on the flux within the SPIRE/FTS beam. Neither option is free from possible systematics. However, for a mid- $J$ CO line, (a) is likely a better option because both the $70 \mu \mathrm{m}$ dust emission and the warm CO line emission originate from more or less the same region, while the spectral energy distribution of the dust emission behind the value of $L_{\mathrm{IR}}$ or $L_{\mathrm{FIR}}$ is expected to vary significantly from the nucleus to the outer disk of a star-forming galaxy. In the remainder of this paper, we use the point-source-calibrated line fluxes in all analyses, but only include those galaxies with $f_{70 \mu \mathrm{m}}(\theta) \geqslant 80 \%$ when we consider a line-to-IR luminosity ratio where $\theta=30^{\prime \prime}$ or $17^{\prime \prime}$, depending on whether the line is covered in the SLW or SSW segment. In this manner the line fluxes used will always be less than $20 \%$ underestimated compared to their actual value.

\subsection{Line Detection Rate}

Panels (b) to (n) in Figure 8 are plots of the fractional detection rates of the spectral lines tabulated in Table 4 in a number of bins of $F_{\mathrm{IR}}$, indexed numerically from 1 to 7 . The bins are delineated at the following flux values: 7.5, 10, 15, 22, 35 , and $120 \times 10^{-13} \mathrm{~W} \mathrm{~m}^{-2}$. For example, the first bin of index 1 is for $6.5 \leqslant F_{\mathrm{IR}}<7.5 \times 10^{-13} \mathrm{~W} \mathrm{~m}^{-2}$, the second bin for $7.5 \leqslant F_{\mathrm{IR}}<10 \times 10^{-13} \mathrm{~W} \mathrm{~m}^{-2}$, and the last bin of index 7 for $F_{\mathrm{IR}}>120 \times 10^{-13} \mathrm{~W} \mathrm{~m}^{-2}$. Only the detections with a quality flag of $Q=1$ and 2 are used here. As a comparison, panel (a) in Figure 8 shows the number distribution of the observed LIRGs, with the galaxy number in each flux bin marked explicitly. It is clear that we have achieved a detection completeness of better than $\sim 90 \%$ at all flux levels for $\mathrm{CO}(6$ $-5), \mathrm{CO}(7-6)$, [C I] $370 \mu \mathrm{m}$, and [N II] $205 \mu \mathrm{m}$. The next best cases are for $\mathrm{CO}(5-4)$ and $\mathrm{CO}(8-7)$, for which the detection rates are still more than $\sim 60 \%$ even in the faintest flux bins.

Figure 9 shows similar plots for selected fainter spectral lines based on the detections tabulated in Table 5. It is clear that the detection rate is rather low for all of these lines, except in the three brightest flux bins, where the number of galaxies is low. To alleviate this shortcoming, we use stacked spectra created 
Table 5

Additional Detected Lines

\begin{tabular}{|c|c|c|c|c|c|c|c|c|c|}
\hline $\begin{array}{l}\text { Name } \\
\text { (1) }\end{array}$ & $\begin{array}{l}\text { Line } \\
\text { (2) }\end{array}$ & $\begin{array}{l}F^{\mathrm{a}} \\
(3)\end{array}$ & $\begin{array}{l}\sigma_{\mathrm{F}}^{\mathrm{b}} \\
(4)\end{array}$ & $\begin{array}{c}\nu_{\mathrm{obs}}{ }^{\mathrm{c}} \\
\text { (5) }\end{array}$ & $\begin{array}{l}\sigma_{\nu}{ }^{\mathrm{d}} \\
(6)\end{array}$ & $\begin{array}{c}\nu_{\text {diff }}^{\mathrm{e}} \\
(7)\end{array}$ & $\begin{array}{c}\left(f_{\nu}\right)_{\mathrm{p}}{ }^{\mathrm{f}} \\
(8)\end{array}$ & $\begin{array}{c}(\mathrm{S} / \mathrm{N})^{\mathrm{g}} \\
(9)\end{array}$ & $\begin{array}{c}Q^{\mathrm{h}} \\
(10)\end{array}$ \\
\hline \multirow[t]{4}{*}{ NGC 0023} & $\mathrm{H}_{2} \mathrm{O}\left(3_{21}-3_{12}\right)$ & -1.53 & 0.17 & 1145.41 & 0.09 & -0.06 & -1.29 & 4.03 & 2 \\
\hline & $\mathrm{HF}(1-0)$ & -1.39 & 0.21 & 1213.74 & 0.10 & -0.24 & -1.17 & 4.50 & 2 \\
\hline & $\mathrm{HCN}(16-15)$ & 1.14 & 0.21 & 1395.89 & 0.13 & 0.46 & 0.96 & 3.20 & 2 \\
\hline & HCN (17-16) & 1.56 & 0.24 & 1483.76 & 0.10 & 1.31 & 1.32 & 3.30 & 4 \\
\hline \multirow[t]{5}{*}{ NGC 0034} & $\mathrm{H}_{2} \mathrm{O}\left(2_{02}-1_{11}\right)$ & $1.01 \mathrm{~S}$ & 0.12 & 969.36 & 0.08 & 0.44 & 0.85 & 7.73 & 1 \\
\hline & $\mathrm{H}_{2} \mathrm{O}\left(3_{12}-3_{03}\right)$ & 0.49 & 0.07 & 1076.35 & 0.10 & 0.10 & 0.42 & 4.20 & 2 \\
\hline & $\mathrm{H}_{2} \mathrm{O}\left(3_{21}-3_{12}\right)$ & 0.73 & 0.07 & 1140.87 & 0.06 & 0.33 & 0.61 & 3.59 & 2 \\
\hline & $\mathrm{H}_{2} \mathrm{O}^{+}\left(1_{11}-0_{00} \mathrm{~J}=3 / 2-1 / 2\right)$ & 0.37 & 0.08 & 1094.60 & 0.13 & 0.85 & 0.31 & 3.88 & 2 \\
\hline & $\mathrm{OH}^{+}\left(1_{12}-0_{12}\right)$ & 0.58 & 0.09 & 1013.18 & 0.10 & -0.07 & 0.49 & 3.77 & 2 \\
\hline
\end{tabular}

Notes.

${ }^{a}$ Line flux in $10^{-17} \mathrm{~W} \mathrm{~m}^{-2}$. A negative value here indicates an absorption. Note that the flux derivation assumed a point-source case. See Section 4.2 for a prescription of the flux aperture correction in case the target is moderately extended with respect to the SPIRE/FTS beam. The flux values of the $\mathrm{OH}^{+}\left(1_{22}-0_{11}\right)$ and $\mathrm{H}_{2} \mathrm{O}$ $\left(2_{02}-1_{11}\right)$ lines end with suffix "S" (standing for SSW) or "L" (SLW) to indicate from which detector array the flux was measured.

${ }^{\mathrm{b}}$ Line flux uncertainty in $10^{-17} \mathrm{~W} \mathrm{~m}{ }^{-2}$.

${ }^{\mathrm{c}}$ Observed line frequency in GHz.

${ }^{\mathrm{d}}$ Uncertainty of the observed line frequency in GHz.

e This equals the observed frequency minus the expected line frequency based on the redshift of the target, expressed in units of GHz.

${ }^{\mathrm{f}}$ Peak line flux density in Jy. A negative value here also indicates an absorption.

${ }^{\mathrm{g}} \mathrm{S} / \mathrm{N}$ for the peak line flux density.

${ }^{\mathrm{h}}$ This is the same quality flag as defined in Table 4.

(This table is available in its entirety in machine-readable form.)

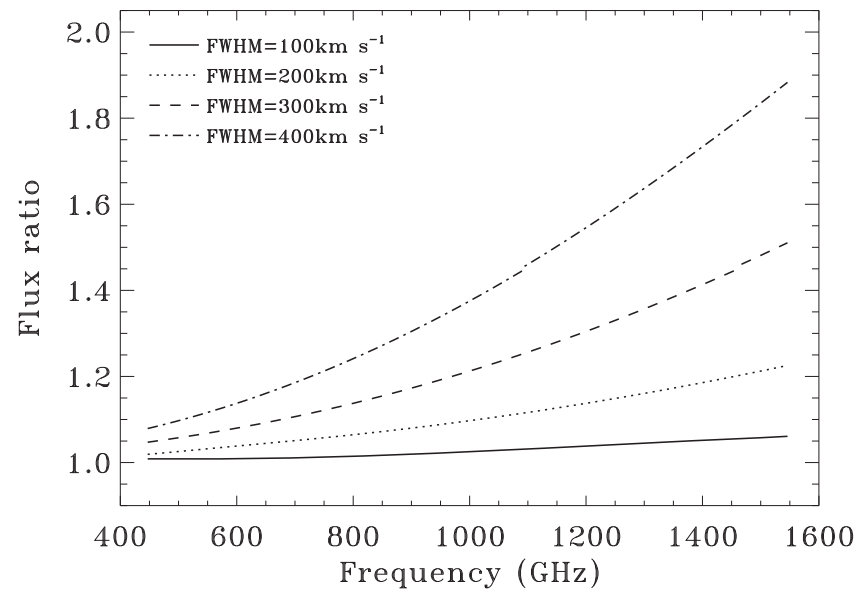

Figure 5. Theoretically predicted ratio of the flux of a sinc-Gaussian line profile to the flux of a sinc-only line profile as a function the line central frequency. The width of the sinc function in each line profile is fixed at the SPIRE/FTS spectral resolution. The results are shown for four different FWHM values (as noted in the legend) of the Gaussian component of the sincGaussian profile. Both line profiles have the same central frequency and peak flux density.

by summing over the spectra of individual sources, to study some of these fainter lines.

\section{Data Analysis and Discussion}

\subsection{CO SLEDs}

Figure 10 is a comparison of the observed CO SLEDs among the brightest galaxies in each of the following 3 FIR color bins: (a) 11 "FIR-cold" galaxies (in red) of $0.50<C(60 /$

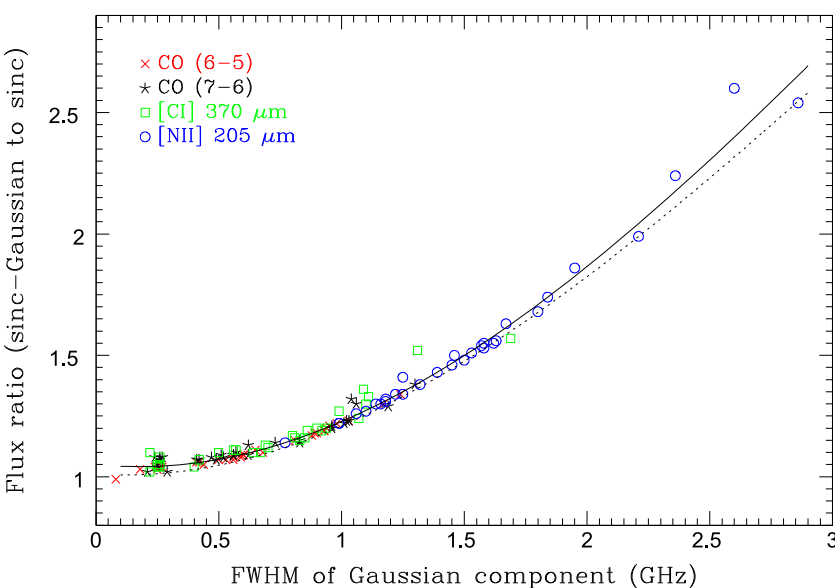

Figure 6. Plot of the line flux ratio of a line fit with a sinc-Gaussian profile to the same line fit with a sinc-only profile as a function of the fitted FWHM (in $\mathrm{GHz}$ ) of the Gaussian component for the [N II] line, $\mathrm{CO}(7-6), \mathrm{CO}(6-5)$, and [C I] $370 \mu \mathrm{m}$ detected at high $\mathrm{S} / \mathrm{Ns}$ in 31 archival spectra. The different spectral lines are color-coded, as explained in the legend. The dotted curve is a third-order polynomial representation of the theoretical prediction from Figure 2. The solid curve is a third-order polynomial fit to the data points here. The overall vertical rms residual between the data points and the solid curve is 0.03

$100) \leqslant 0.65$, which are brighter than $f_{\nu}(60 \mu \mathrm{m})=8.1 \mathrm{Jy}$; (b) 11 "FIR-intermediate" galaxies (in black) of $0.75<C(60 /$ $100) \leqslant 0.90$ and brighter than $f_{\nu}(60 \mu \mathrm{m})=12.0 \mathrm{Jy}$; and (c) 10 "FIR-warm" galaxies (in blue) of $C(60 / 100) \geqslant 1.0$ and brighter than $f_{\nu}(60 \mu \mathrm{m})=10.7 \mathrm{Jy}$.

These represent the brightest sample galaxies in their respective FIR color bin. All satisfy $f_{70 \mu \mathrm{m}}\left(17^{\prime \prime}\right)>0.8$, which means that the shape of each CO SLED should not deviate by 


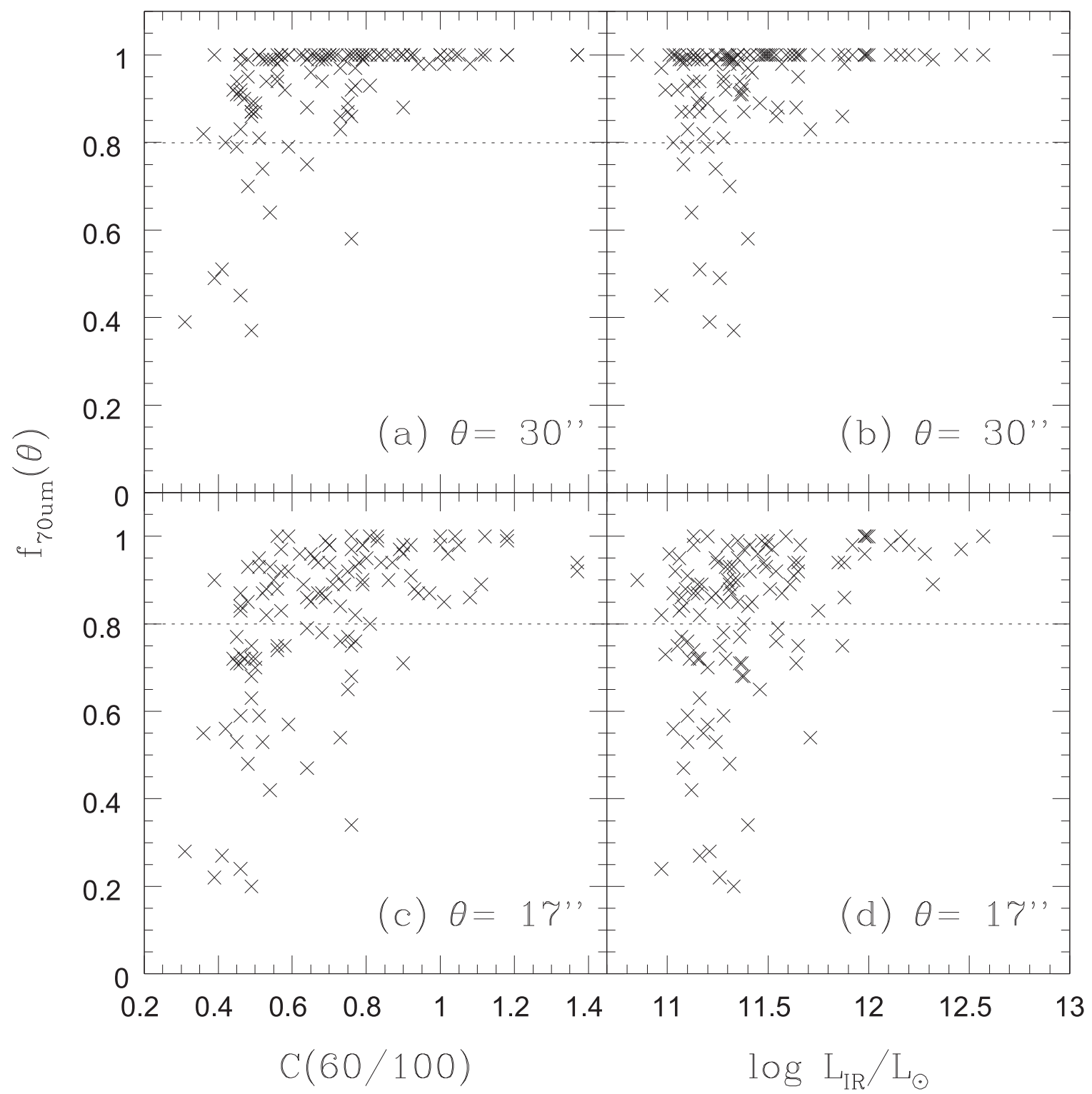

Figure 7. Plots of $f_{70 \mu \mathrm{m}}(\theta)$, the fractional $70 \mu \mathrm{m}$ flux within a Gaussian beam of FWHM $\theta$, as a function of $C(60 / 100)$ or $L_{\mathrm{IR}}$ for $\theta=30^{\prime \prime}$ (top panels) and $17^{\prime \prime}$ (bottom panels). The dotted line in each plot indicates $f_{70 \mu \mathrm{m}}(\theta)=0.8$.

more than $20 \%$ from the true shape across the plotted range of $J$ values, even if the target is not point-like with respect to the SPIRE/FTS beam sizes in SSW. All the CO SLEDs in Figure 10 are normalized to unity at $J=6$. They are arranged from the top left to bottom right in an increasing order of $L_{\mathrm{IR}}$. These plots together reinforce the previous finding ( $\mathrm{Lu}$ et al. 2014; Rosenberg et al. 2015) that the overall shape of a CO SLED is more fundamentally correlated with the FIR color than with $L_{\mathrm{IR}}$. For example, NGC 4418 has only a modest $L_{\mathrm{IR}}$, but one of the warmest FIR colors known in the local universe. Its CO SLED appears even "warmer" than that of Mrk 231, which is more than 10 times more IR luminous. Since the FIR color is driven in turn by the spatially averaged intensity of the dust heating radiation field (e.g., Draine \& Li 2007), this finding suggests that it is the radiation field intensity that shapes the overall CO excitation condition over $4<J<13$.

A few CO SLEDs in Figure 10 show an apparent "kink," with the integrated line flux of a CO line (most often CO (9 $-8)$ ) being lower than the value from a smooth interpolation from the neighboring data points. Such examples include Mrk 231, IRAS F05189-2524, and ESO 286-IG 019. However, these "out-of-line" data points are mostly at a significance no greater than $3 \sigma$ based on the error bars shown here. Furthermore, the $\mathrm{CO}(9-8)$ line is near the long-wavelength end of SSW. As we mentioned before, the error bars plotted here (from Table 4, Row 2 in Column 7) are from the linefitting procedure and might somewhat underestimate the true line flux uncertainty for $\mathrm{CO}(9-8)$. Therefore, this $\mathrm{CO}(9-8)$ kink phenomenon is likely to be an artifact.

In Figure 11 we plot the individual CO SLEDs from Figure 10 separately for the three FIR color bins. The individual CO SLEDs are plotted in various colors. For clarity, only the detected lines are included. Within each FIR color bin, we derived a median flux value at each $J$ using the detected lines only and also plotted the "median CO SLED" synthesized from these median flux values as a thick black curve. Since the upper limits were not used to calculate these median values, some caution should be exercised when interpreting the 


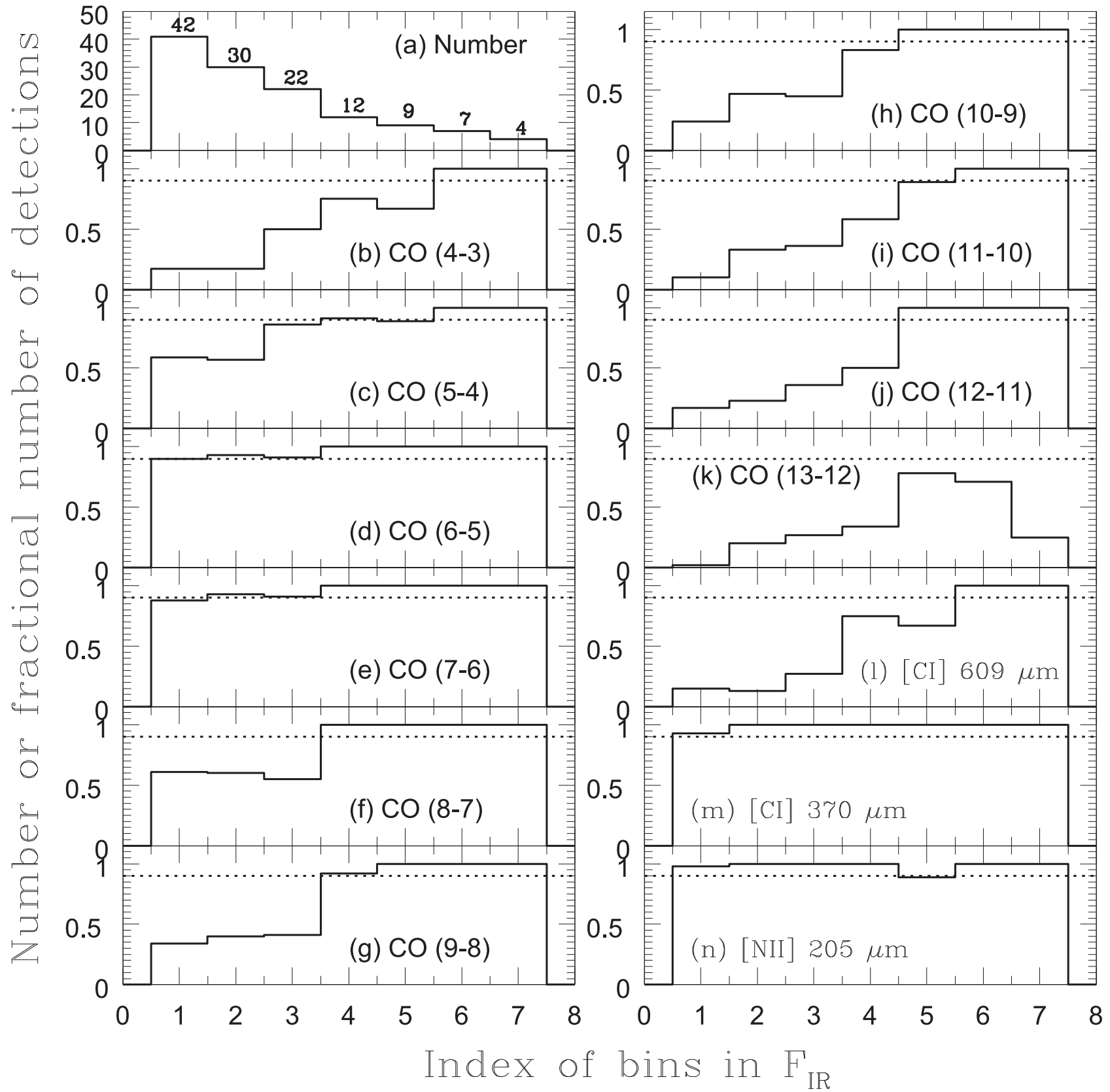

Figure 8. Panels (b) to (n) show the fractional detection rates of the main targeted spectral lines in a number of bins of the total IR flux $F_{\mathrm{IR}}$, where the flux bins are delineated at the following values: 7.5, 10.0, 15.0, 22, 35, and $120 \times 10^{-13} \mathrm{~W} \mathrm{~m}^{-2}$. For example, the first bin (i.e., index $=1$ ) has $6.5 \leqslant F_{\mathrm{IR}}<7.5 \times 10^{-13} \mathrm{~W} \mathrm{~m} \mathrm{~m}^{-2}$, the second bin has $7.5 \leqslant F_{\mathrm{IR}} \leqslant 10 \times 10^{-13} \mathrm{~W} \mathrm{~m}^{-2}$ and the last bin is for $F_{\mathrm{IR}}>120 \times 10^{-13} \mathrm{~W} \mathrm{~m}^{-2}$. The subject spectral line and the $90 \%$ completeness level are marked in each plot. As a comparison, panel (a) shows similar distributions of the total observed galaxies, with the number of galaxies in each flux bin explicitly marked. The multiple entries for NGC 3690 and NGC 2146 in Table 1 were counted separately here.

significance of a median flux value when there are many upper limits, e.g., near the high-J end in Figure 11(a). Nevertheless, these median CO SLEDs, given numerically in Table 6, are still useful for illustrating the systematic changes in the shapes of CO SLEDs as the FIR color increases. There is also an indication that the variance of the CO SLED shapes also increases as the FIR color increases. Since all the CO SLEDs are normalized to 1 at $J=6$, this variance manifests itself in the scatter of the individual CO SLEDs at the high- $J$ end in each plot. In particular, a large variance is seen in the warmest FIR color bin. This may suggest that the local condition of the radiation field and gas density become so complicated or extreme that the spatially averaged FIR color becomes less accurate at predicting the shape of a CO SLED. In Figure 11(c) we have labelled the three individual galaxies associated with some of the warmest CO SLEDs in our sample. One of them, NGC 4418, is known to be among the most compact extragalactic IR sources known (e.g., Sakamoto et al. 2013).

To confirm that the systematic variation in SLED shape seen for the brightest galaxies of the sample in Figures 10 and 11 is not dependent on their $60 \mu \mathrm{m}$ flux densities, we show the entire sample in Figure 12 by plotting each CO line luminosity, normalized by that of $\mathrm{CO}(6-5)$, as a function of the FIR color. For $J=4$ or $J \gtrsim 9$, there are now significant numbers of nondetections. We therefore focus on the overall trend and whether the upper limits are consistent with the trend. To this end, in each 


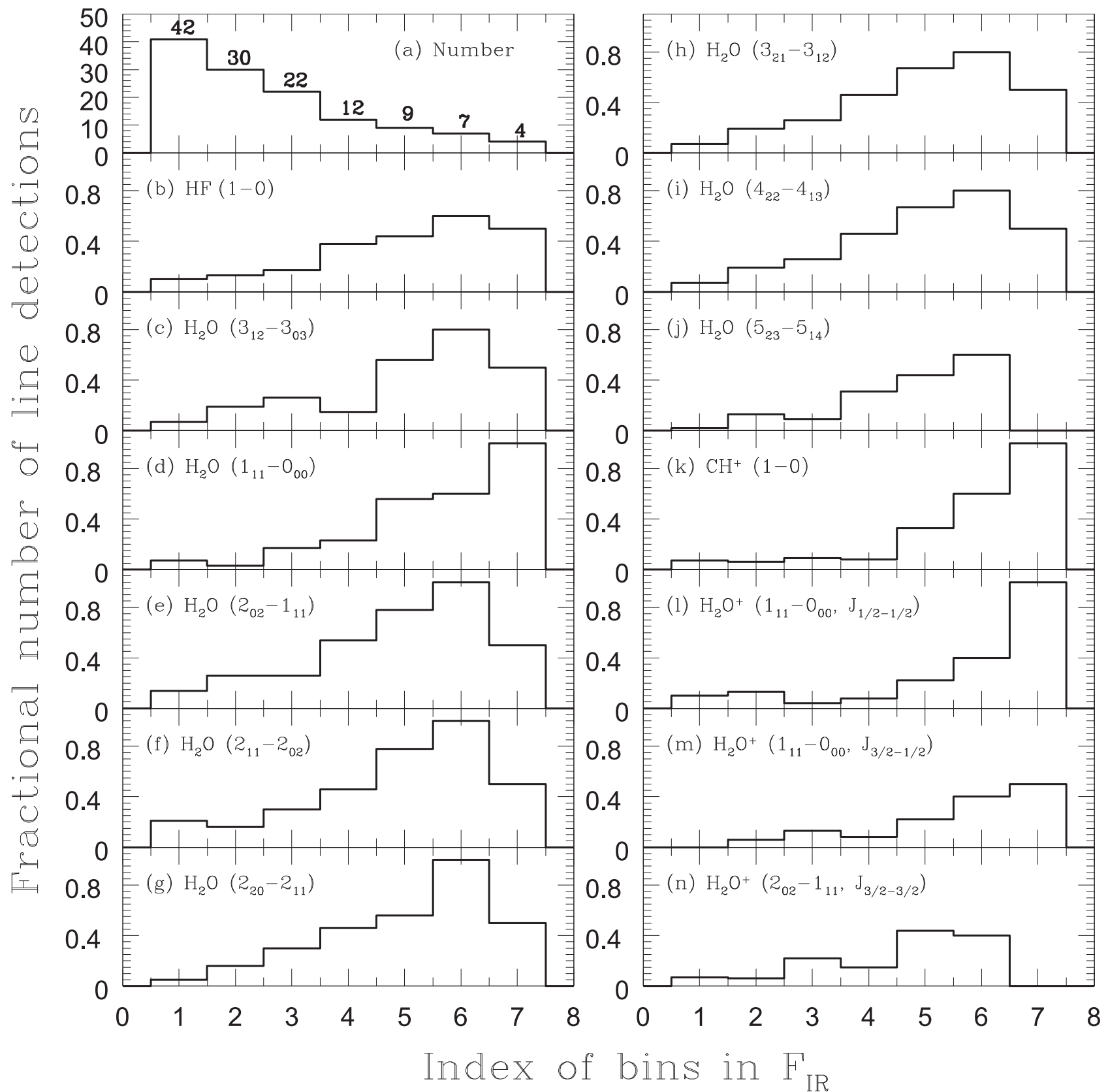

Figure 9. Plots similar to those in Figure 8, but for the fractional detection (in either emission or absorption) rates of selected fainter spectral lines. The subject spectral line is labeled in each plot.

plot the horizontal dotted line marks where the normalized line flux equals 1 and the two vertical dashed lines separate the three color bins used in Figures 10 and 11. The individual sources belonging to the dominant AGN subsample defined earlier are shown in red. For the CO lines of $J \geqslant 9$ (covered in SSW), we only used those sources with $f_{70 \mu \mathrm{m}}\left(17^{\prime \prime}\right) \geqslant 0.8$ in order to control the effect on the normalized line fluxes from the SPIRE/ FTS beam size difference between SSW and SLW. For $C(60 /$ $100) \lesssim 0.7$, the CO SLEDs tend to be brightest at $J<6$. In contrast, at $C(60 / 100) \sim 1.0$, the observed CO SLEDs become nearly flat across all $J$ levels or even show somewhat increasing normalized line fluxes toward higher $J$. At the FIR colors in between, the CO SLEDs tend to peak around $J \sim 6$ or 7 . These observations are all consistent with the data of the brightest sample galaxies shown in Figures 10 and 11.
Furthermore, there is no obvious segregation between the dominant AGNs and the rest of the sample in any plot in Figure 12. With the caveat that our AGN sample size is small, this implies that the presence of an energetic AGN in a LIRG has little or only marginal effect on the shape of the CO SLED over the $J$ levels shown here. This is in general agreement with other recent studies on the mid- $J$ CO line emissions in individual AGNs (e.g., Pereira-Santaella et al. 2013; Zhao et al. 2016b). We discuss the subject of AGN gas heating in more detail in Section 5.3.

\subsection{Star Formation and Molecular Gas Heating}

The simplest model for explaining the systematic change in the shapes of the CO SLEDs shown in the previous figures is to 

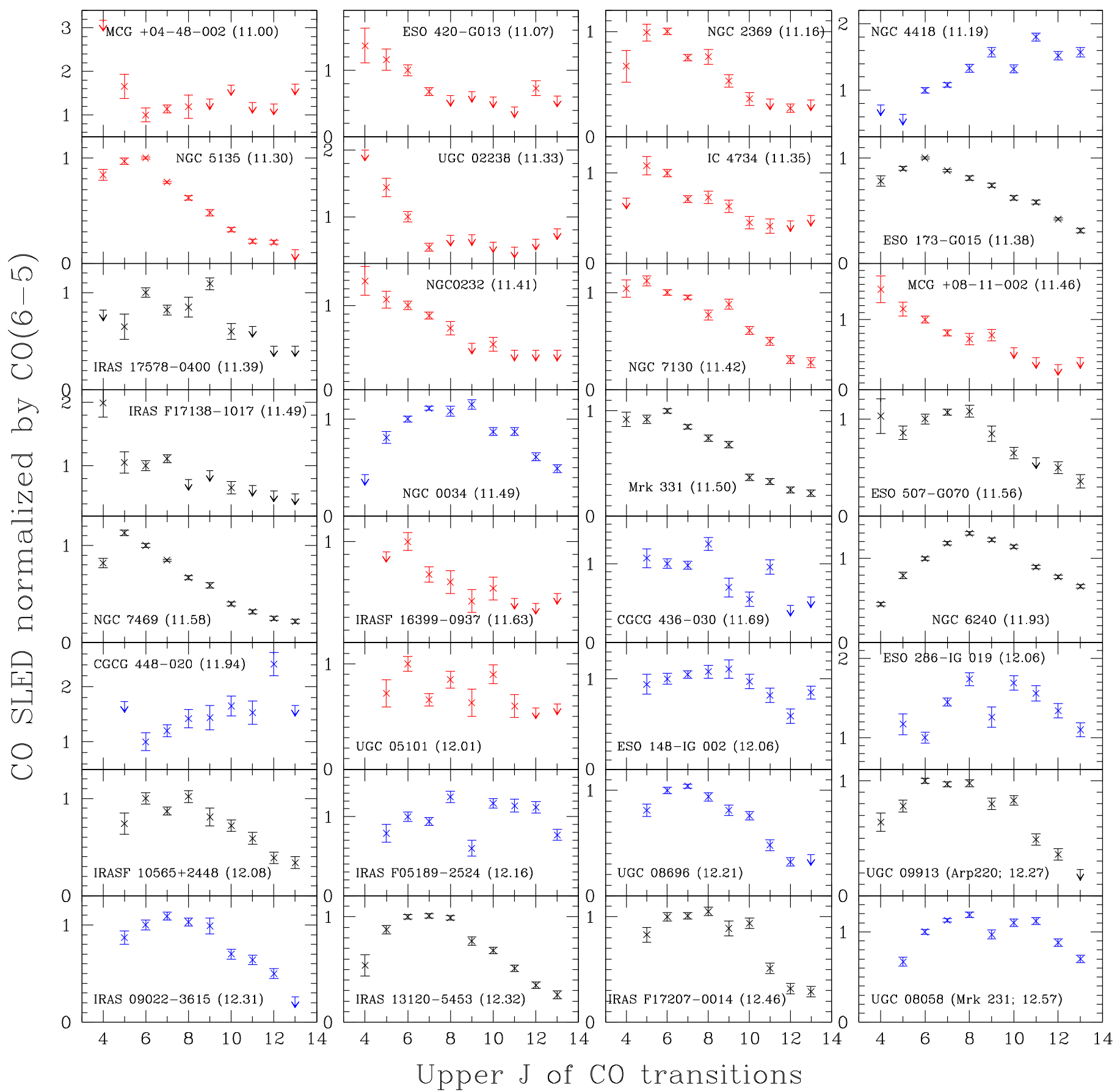

Figure 10. Individual CO SLEDs, each normalized to 1 at $J=6$, of the brightest sample galaxies in each of the three FIR color bins: Those in red are for the 11 FIRcold galaxies with $0.50<C(60 / 100) \leqslant 0.65$ and $f_{\nu}(60 \mu \mathrm{m})>8.1 \mathrm{Jy}$, those in black are for the 11 galaxies with $0.75<C(60 / 100) \leqslant 0.90$ and $f_{\nu}(60 \mu \mathrm{m})>12.0 \mathrm{Jy}$, and those in blue are for the 10 FIR-warm galaxies with $C(60 / 100) \geqslant 1.0$ and $f_{\nu}(60 \mu \mathrm{m})>10.7 \mathrm{Jy}$. All the galaxies shown here satisfy $f_{70 \mu \mathrm{m}}\left(17^{\prime \prime}\right)>0.8$. The individual SLEDs are arranged in increasing order of $L_{\mathrm{IR}}$ from the top left to the bottom right. The galaxy name and logarithmic $L_{\mathrm{IR}}$ in solar units (in parentheses) are noted in each panel.

assume a single molecular gas phase that gets warmer and denser when the FIR color becomes warmer. However, we showed earlier ( $\mathrm{Lu}$ et al. 2014) that one generally requires at least two distinct $\mathrm{CO}$ gas phases to explain the observed $\mathrm{CO}$ SLEDs. We explore this important finding further using the full galaxy sample here, which is twice the size of the sample used in Lu et al. (2014).

Figure 13 shows how a CO line luminosity, normalized by $L_{\mathrm{IR}}$, varies as a function of the FIR color for the entire sample. As in Figure 12, those dominant AGNs are shown in red. All the plots in Figure 13 span 2.0 dex vertically to facilitate direct comparison. The horizontal dotted line in each plot indicates the value of -4.88 , the average logarithmic $\mathrm{CO}(7-6) / \mathrm{IR}$ luminosity ratio identified by Lu et al. (2015) on a combined set of the LIRGs from the current sample and additional ULIRGs from the Herschel archive. This line serves as a fiducial level to help identify the most energetic $\mathrm{CO}$ line across all $J$ values at a given $C(60 / 100)$. If one focuses first on the mid- $J$ CO lines of $J=6$ or 7 in Figure 13, the line-to-IR luminosity ratios appear to only weakly depend on $C(60 / 100)$ 


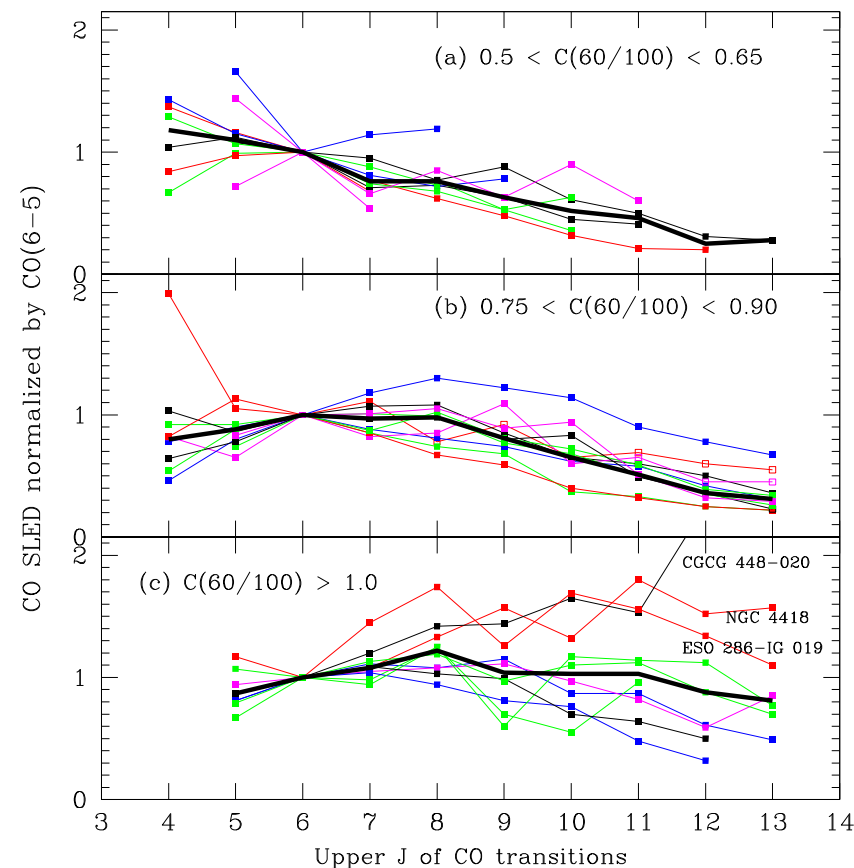

Figure 11. Plots of the individual CO SLEDs (connected squares in various colors) from Figure 10, along with the median CO SLEDs (the thick curve in black) they generate, for each of the three FIR color bins (as labeled in the plots). The three galaxies with the warmest CO SLEDs are labeled in the bottom panel.

Table 6

Median CO SLEDs from Brightest Sample Galaxies

\begin{tabular}{lccc}
\hline \hline $\begin{array}{l}\text { Line } \\
(1)\end{array}$ & $\begin{array}{c}\text { Cold }^{\mathrm{a}} \\
(2)\end{array}$ & $\begin{array}{c}\text { Intermediate }^{\mathrm{a}} \\
(3)\end{array}$ & $\begin{array}{c}\text { Warm }^{\mathrm{a}} \\
(4)\end{array}$ \\
\hline $\mathrm{CO}(4-3)$ & 1.18 & 0.80 & $\ldots$ \\
$\mathrm{CO}(5-4)$ & 1.10 & 0.88 & 0.87 \\
$\mathrm{CO}(6-5)$ & 1.00 & 1.00 & 1.00 \\
$\mathrm{CO}(7-6)$ & 0.76 & 0.97 & 1.08 \\
$\mathrm{CO}(8-7)$ & 0.76 & 0.98 & 1.22 \\
$\mathrm{CO}(9-8)$ & 0.63 & 0.81 & 1.04 \\
$\mathrm{CO}(10-9)$ & 0.52 & 0.65 & 1.03 \\
$\mathrm{CO}(11-10)$ & 0.46 & 0.51 & 1.03 \\
$\mathrm{CO}(12-11)$ & 0.25 & 0.36 & 0.88 \\
$\mathrm{CO}(13-12)$ & 0.28 & 0.31 & 0.81 \\
\hline
\end{tabular}

Note.

a These columns correspond to the same FIR color bins used in Figures 10 and 11: $0.5<C(60 / 100) \leqslant 0.65$ (i.e., "Cold"), $0.75<C(60 / 100) \leqslant 0.9$ ("Intermediate"), and $1.0<C(60 / 100) \leqslant 1.4$ ("warm"). Note that the frequencydependent SPIRE/FTS beam has a minimal effect on the results here, as these results were derived using galaxies satisfying $f_{70 \mu \mathrm{m}}\left(17^{\prime \prime}\right)>0.80$.

and show apparently the smallest scatters among all the lines plotted in Figure 13. In fact, the ratios shown in panel $(J)$ in Figure 13 are for the combined $\mathrm{CO}(6-5)$ and $\mathrm{CO}(7-6)$ line fluxes. Excluding the AGNs and the outlier NGC 6240, the ratios of the remaining galaxies show very little dependence on $C(60 / 100)$ across the entire FIR color range covered here. On the other hand, the first two panels in Figure 13 show that, as $C$ $(60 / 100)$ becomes smaller, $\mathrm{CO}(4-3)$ or even $\mathrm{CO}(5-4)$ becomes relatively stronger. As pointed out in $\mathrm{Lu}$ et al. (2014), a single gas phase model can not explain these observations. One needs at least two gas components: a warm and dense component, which emits the $\mathrm{CO}$ lines primarily in the mid- $J$ (i.e., $5 \lesssim J \lesssim 10$ ) with a general peak around $J \approx 6$ or 7 and correlates energetically with the dust emission, is mainly responsible for the observed constant ratio seen around $J=6$ or 7 . Since the dominant heating source for the IR emission in the majority of LIRGs is unambiguously current $\mathrm{SF}$, the same ongoing SF should also be responsible for this warm $\mathrm{CO}$ gas component, although the exact or dominant heating mechanism is still controversial (see Lu et al. 2014 for a list of possible mechanisms). The other gas component is a cold gas phase of moderate density, which emits $\mathrm{CO}$ lines primarily at $J \lesssim 4$ and is not directly related to current SF that powers $L_{\mathrm{IR}}$ in LIRGs.

This two-component picture has been supported by other independent studies, with many of the recent studies on nonLTE modeling of observed CO SLEDs in LIRGs and starforming galaxies pointing to at least two distinct gas phases (e.g., Panuzzo et al. 2010; Van der Werf et al. 2010; Rangwala et al. 2011; Kamenetzky et al. 2012; Spinoglio et al. 2012; Pellegrini et al. 2013; Pereira-Santaella et al. 2013; Rigopoulou et al. 2013; Papadopoulos et al. 2014; Rosenberg et al. 2014a, 2014b; Schirm et al. 2014; Xu et al. 2015). A few of our sample galaxies have also been directly imaged in $\mathrm{CO}(6-5)$ at a high angular resolution. The resulting $\mathrm{CO}(6-5)$ line emission shows a very different spatial scale than either $\mathrm{CO}(1-0)$ or CO (2-1) (e.g., Xu et al. 2014, 2015). This further supports our two-component picture.

\subsection{AGN and Molecular Gas Heating}

We showed in Figure 12 that the presence of a dominant AGN in a galaxy does not appear to have a significant impact on the shape of a CO SLED up to $J \sim 10$, and in Figure 13 that most of the dominant AGNs in our sample tend to show a lower mid-J CO-to-IR ratio. We explore the possible physics behind these phenomena here.

Figure 14 is a plot of the $\log$ of the ratio of the CO line luminosity summed over $\mathrm{CO}(6-5)$ and $\mathrm{CO}(7-6)$ to $L_{\mathrm{IR}}$ as a function of $f_{\mathrm{AGN}}$ for the same set of galaxies shown in Figure 13(j). We have offset the $Y$-axis by the sample median $\log$ value of -4.53 for the SF-dominated galaxies. The colorcoding scheme is the same as in Figure 13, except for the upper limits shown here in green for the sake of visual clarity. The average $f_{\mathrm{AGN}}$ value and its uncertainty (=the standard deviation of the mean) are plotted as an error bar here, as described in Section 2.2, from Díaz-Santos et al. (2017). The distribution of the data points in Figure 14 indicates a possible trend that points to a lower $Y$-axis value on average as $f_{\mathrm{AGN}}$ increases. For example, the $Y$-axis median value is near zero for the sources with $f_{\mathrm{AGN}} \leqslant 0.25$. This median value would be about -0.1 over $0.25<f_{\mathrm{AGN}} \leqslant 0.5$ if the two upper limits in this bin were also taken into consideration. For $f_{\mathrm{AGN}}>0.5$, there are 5 data points. The resulting median value is -0.31 , equal to about $2.6 \sigma$, where $\sigma$ is the rms scatter of the sample sources with $f_{\mathrm{AGN}}<0.25$. We explain in detail the solid curves in Figure 14 below.

Another useful way to gain insights into AGN heating of molecular gas is to compare the CO SLEDs between wellknown AGNs and starbursts by extending their CO SLEDs from our SPIRE/FTS observations to even higher $J$ levels using PACS spectroscopic data. Lu et al. (2014) singled out Mrk $231 \quad\left(f_{\mathrm{AGN}}=0.77\right.$; Díaz-Santos et al. 2017) and NGC $1068\left(f_{\mathrm{AGN}} \gtrsim 0.50\right.$; Telesco \& Decher 1988; Lu et al. 2014) as two representative AGN-dominated galaxies that 

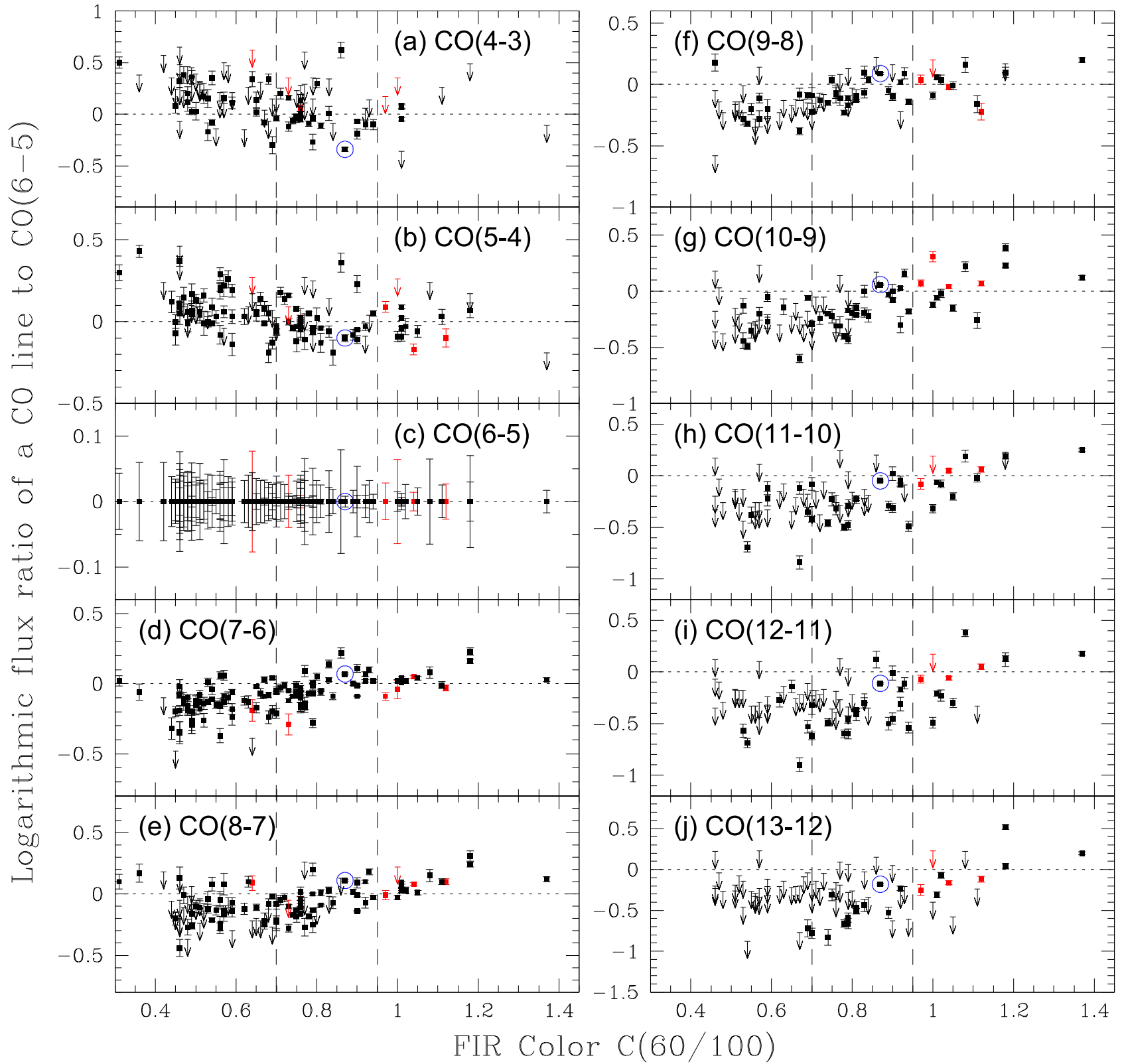

Figure 12. Plots of the $\log$ of the flux ratio of a $\mathrm{CO}$ line of the upper level $J$ to $\mathrm{CO}(6-5)$ as a function of $C(60 / 100)$ for the sample galaxies detected in $\mathrm{CO}(6-5)$ and for $J$ from 4 to 13 (as labeled in each plot). The horizontal dotted line in each plot marks where the logarithmic line ratio equals 0 . The two vertical dashed lines separate the three subsamples used in Figures 10 and 11. For the CO lines covered in SSW (i.e., $J \geqslant 9$ ), we further limited the data plotted here to those galaxies with $f_{70 \mu \mathrm{m}}\left(17^{\prime \prime}\right)>0.8$. The AGNs are shown in red and NGC 6240 is further circled in blue. For the plot of CO (6-5), it shows the relative line flux uncertainty.

display a lower $\mathrm{CO}(7-6)$ to IR ratio than the typical ratios seen in the SF-dominated galaxies in our sample. These AGNs are known to have significant hot $\mathrm{CO}$ gas emissions at $J>10$ (Hailey-Dunsheath et al. 2012; González-Alfonso et al. 2014b), which could be associated with the AGN-powered X-ray dissociated regions (XDR) (Van der Werf et al. 2010; Spinoglio et al. 2012). In Figure 15 we show the expanded CO SLEDs of these two dominant AGNs, along with those of M82, Arp 220 (=UGC 09913), and IC 0694 (= UGC 06472 or Arp 299A), by making use of the PACS spectroscopic data from Mashian et al. (2015). M82 is an archetypical starburst. With $f_{\mathrm{AGN}} \sim 0.12$ (Díaz-Santos et al. 2017), Arp 220 is also dominated by a starburst. The last galaxy, IC 0694, is controversial as to whether it harbors a strong AGN, with both positive evidence for (e.g., Sargent \& Scoville 1991; Della
Ceca et al. 2002; Henkel et al. 2005; Tarchi et al. 2007; Alonso-Herrero et al. 2013; Rosenberg et al. 2014b) and against (e.g., Alonso-Herrero et al. 2000) it. Both Arp 220 and Mrk 231 are compact enough to be practically point sources for both SPIRE and PACS. IC 0694 is itself a compact source and its SPIRE and PACS fluxes were based on a point-source case. The mid-J CO line emission of NGC 1068 is shown to be extended and dominated by the circumnuclear SF based on a SPIRE/FTS mapping observation (Spinoglio et al. 2012). The total SPIRE/FTS CO line fluxes in Table 1 of Spinoglio et al. (2012) were used here. As shown in Spinoglio et al. (2012) and Mashian et al. (2015), the PACS part of the CO SLED is dominated by the central compact source. M82 is somewhat extended with respect to both SPIRE and PACS beams (Kamenetzky et al. 2012; Mashian et al. 2015). Its PACS line 


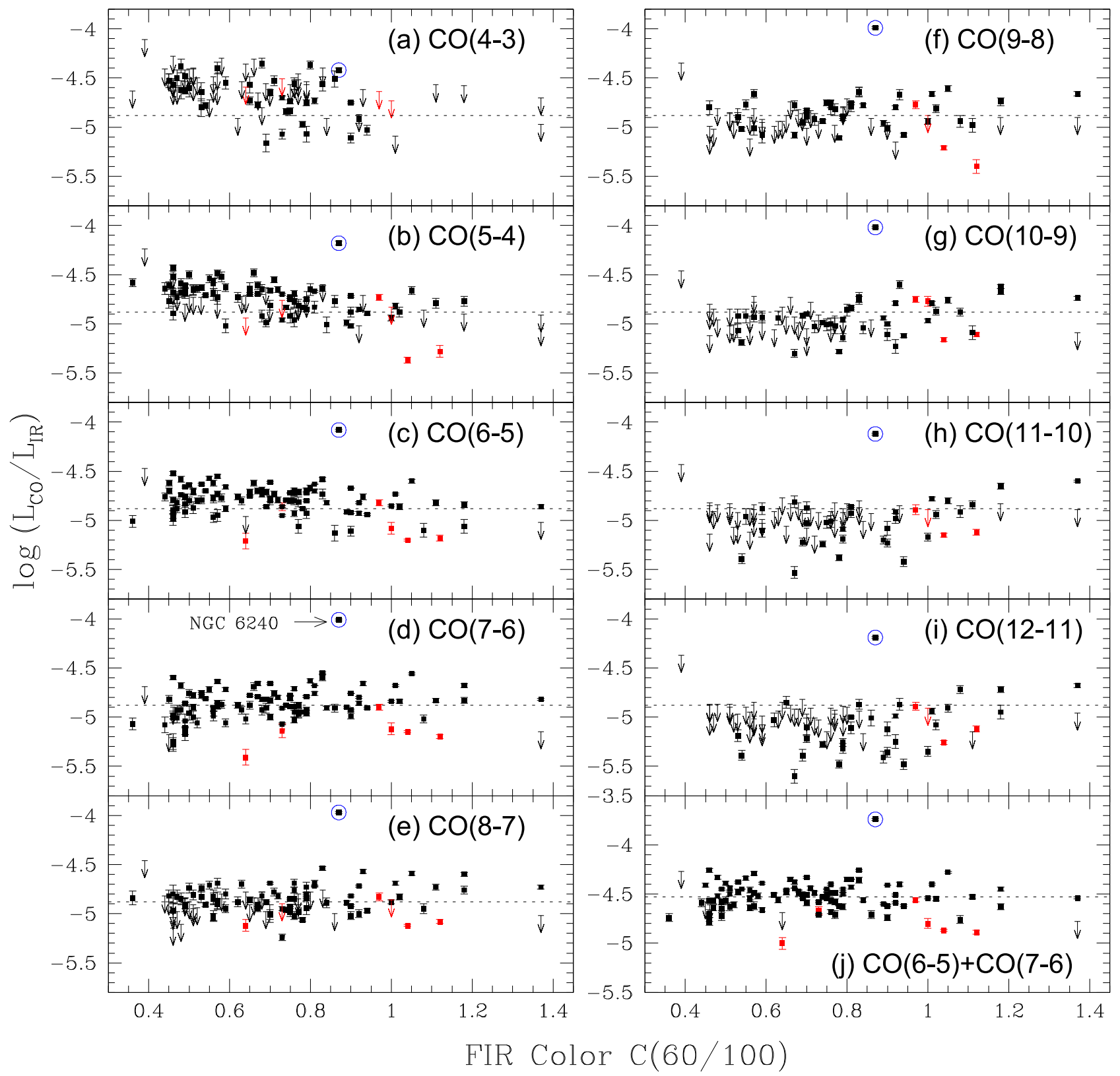

Figure 13. Panels (a) to (i) are plots of the $\log$ of the luminosity of a CO line of the upper level $J$, divided by $L_{\mathrm{IR}}$, as a function of $C(60 / 100)$ for our sample galaxies and for $J$ from 4 to 12 , respectively. For $J<9$ (i.e., with the CO line detected in the SLW array), only the targets with $f_{70 \mu \mathrm{m}}\left(30^{\prime \prime}\right)>0.8$ are plotted; for $J \geqslant 9$ (i.e., detected in SSW), only those with $f_{70 \mu \mathrm{m}}\left(17^{\prime \prime}\right)>0.8$ are shown here. The red data points are the dominant AGNs that also satisfy our FTS beam size-based selection criterion. The galaxy NGC 6240 is further circled in blue. Panel (j) is for the sum of CO (6-5) and CO (7-6). The horizontal dotted lines in panels (a) to (i) indicate the average logarithmic ratios of -4.88 adopted for $\mathrm{CO}(7-6)$ in Lu et al. (2015). The dotted line in (j) indicates a value of -4.53 , the median ratio for the galaxies plotted in (j), excluding the AGNs and NGC 6240.

fluxes were integrated over the PACS field of view of $47^{\prime \prime} \times 47^{\prime \prime}$. We therefore used the SPIRE/FTS CO line fluxes within a constant aperture of comparable size from Kamenetzky et al. (2012).

If one focuses on the mid- $J$ regime, there is an apparent scatter in CO SLED shape between the individual sources. In particular, the CO SLED shape of NGC 1068 appears to be different from that of any of the other sources. However, this could be largely expected given the fact that the mid- $J$ CO SLED shape depends on $\mathrm{C}(60 / 100)$ (see Figure 11). NGC 1068 has a relatively mild FIR color at C(60/ $100)=0.76$. In comparison, IC 0694, M82, and Mrk 231 all have very warm FIR colors, with C $(60 / 100)=1.01,1.08$, and
1.04 , respectively. With $\mathrm{C}(60 / 100)=0.90$, Arp 220 falls in between. As a result, at least part of the mid- $J$ differences among the galaxies seen in Figure 15 should be due to their FIR color differences. In fact, if we "convert" the observed CO SLEDs of NGC 1068 and Arp 220 to what one would have seen if they had $\mathrm{C}(60 / 100)>1.0$, the results would be those shown in the insert in Figure 15. This conversion was done by multiplying the observed SLED by the ratio of two of the median CO SLEDs in the 3 FIR color bins in Figure 11 (or Table 6) based on the FIR color of the target. For Arp 220, this ratio equals Col. (4) divided by Col. (3) in Table 6; for NGC 1068, it is Col. (4) divided by the average value of Cols. (2) and (3) in Table 4, as its FIR color falls between the 


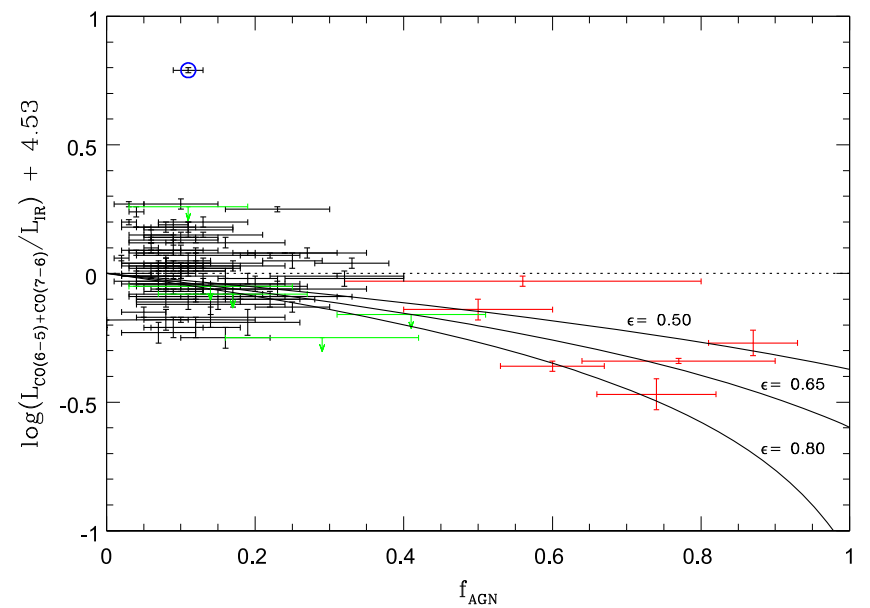

Figure 14. Plot of the logarithmic ratio of the luminosity of the sum of the $\mathrm{CO}(6-5)$ and $\mathrm{CO}(7-6)$ line emission to $L_{\mathrm{IR}}$ as a function of $f_{\mathrm{AGN}}$ for the same set of galaxies as in Figure 13(j). The luminosity ratios plotted are offset by the sample median log value of -4.53 marked in Figure 13(j). The colorcoding scheme is the same as in Figure 13, except for the upper limits shown here in green for clarity. A few galaxies without an $f_{\mathrm{AGN}}$ value are not plotted here. The solid curves stand for the function of $\log \left(1-1.15 \epsilon f_{\mathrm{AGN}}\right)$ with $\epsilon=50 \%, 65 \%$ and $80 \%$, respectively, which are further explained in the text.

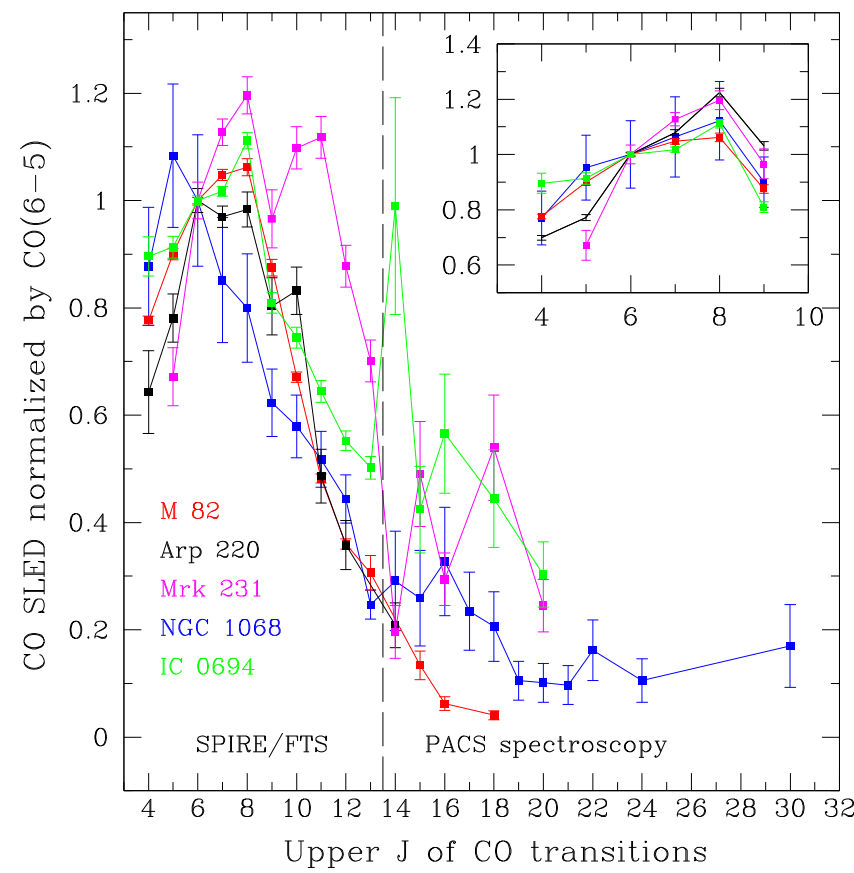

Figure 15. CO SLEDs, all normalized to 1 at $J=6$, of a few well-known starbursts and AGNs (as labeled in the plot). These were constructed by combining the SPIRE/FTS data in this paper (or from the literature in the cases of M82 and NGC 1068) with the higher- $J$ CO line fluxes from the PACS data in Mashian et al. (2015). The vertical dashed line separates the SPIRE/FTS frequency side from the PACS side. The small insert shows the sections of these CO SLEDs over $4 \leqslant J<10$, after "removing" a systematic dependence of the mid- $J$ CO SLED shape on the FIR color. (See the text for more details.)

FIR color ranges associated with the latter two columns. This exercise illustrates an important point of Figure 15: (A) there is no significant (systematic) difference over $5 \lesssim \mathrm{J} \lesssim 10$ (i.e., the mid- $J$ regime) between the AGNs and starbursts considered here after one has accounted for the FIR color dependence of the mid- $J$ CO SLED shape. Another important point of Figure 15 is that (B) the two dominant AGNs, Mrk 231 and NGC 1068, show significant excess of the CO line emission at higher $J$ (i.e., $J>13$ ) in comparison with the two starburstdominated galaxies, M82 and Arp 220.

(A) is the same conclusion we drew in Figure 12, i.e., the mid- $J$ CO SLED shape is not impacted by the presence of an AGN in any obvious and significant way. There are two possible explanations for (A): (i) that SF and AGN gas heating produce more or less the same CO SLED shape in the mid- $J$ regime, or (ii) that the gas cooling associated with AGN gas heating occurs mainly at $J>10$. We reject scenario (i) with different arguments, depending on whether the observed gas cooling at $J>10$ is due to AGN heating. If the observed gas cooling at $J>10$ is due to AGN heating, this implies that the dense $\mathrm{CO}$ gas surrounding the AGN is very hot, no matter whether the actual gas heating is via the $\mathrm{X}$-ray radiation alone or via a combined X-ray and far-UV radiation field from the AGN. Therefore, it is unrealistic for the mid- $J$ CO SLED shape to be similar to that in a pure starburst case. On the other hand, if the observed gas cooling at $J>10$ were not related to AGNs, most of the gas cooling associated with AGN heating would be limited to being within the mid- $J$ regime. In this case, the lower mid-J CO-to-IR flux ratios of AGNs seen in Figures 13 or 14 would conflict with the fact that AGNs should be more effective in heating the gas than the dust, regardless of whether it is via X-ray or AGN-driven shocks, leading to a mid- $J$ CO-to-IR flux ratio higher than those of SFdominated galaxies. Therefore (ii) is the most likely explanation. Namely, the lower mid- $J$ CO-to-IR luminosity ratios associated with those dominant AGNs are due to the fact that the SPIRE/FTS spectral coverage does not sample most of the $\mathrm{CO}$ gas cooling associated with the AGN heating. This conclusion is also consistent with the theoretical prediction (e.g., Spaans \& Meijerink 2008) that gas temperatures in XDRs can be much higher than those found photon-dominated regions (PDRs) powered by the far-UV radiation of young, massive stars.

The conclusion drawn above leads to two important corollaries. One is that the mid- $J$ CO line emission of a (U) LIRG is entirely powered by SF, regardless of whether an AGN is present or not. This lays the foundation for a mid- $J$ CO line, such as $\mathrm{CO}(7-6)$, to be used as a robust SFR tracer, both locally and at high redshifts ( $\mathrm{Lu}$ et al. 2015). The other corollary is that, as shown in Lu et al. (2014), the expected $Y$ axis position of an AGN in Figure 14 is given by

$$
\delta[\log (\mathrm{CO} / \mathrm{IR})]=\log \left(1-L_{\mathrm{IR}}^{\mathrm{AGN}} / L_{\mathrm{IR}}\right),
$$

where $L_{\mathrm{IR}}^{\mathrm{AGN}}$ is the IR luminosity attributed to the AGN (i.e., excluding the SF in the host galaxy). To relate Equation (3) to $f_{\mathrm{AGN}}$, one has to relate the two IR luminosities on the right side of the equation to their respective bolometric luminosities. It is relatively secure to establish (via observations) a mean relationship between $L_{\mathrm{IR}}$ and the bolometric luminosity, $L_{\text {bolo }}$, for the galaxy as a whole. Veilleux et al. (2009) proposed an average of $L_{\mathrm{bolo}} / L_{\mathrm{IR}}=1.15$ for ULIRGs. We adopt this ratio also for our LIRGs. In contrast, it is not observationally straightforward to establish such a relationship for the AGN itself, i.e., separating the AGN from its host galaxy. This would require high spatial resolution observations in the mid- to farinfrared. If we denote the quantity $L_{\mathrm{IR}}^{\mathrm{AGN}} / L_{\mathrm{bolo}}^{\mathrm{AGN}}$ by $\epsilon$, then we have

$$
\delta[\log (\mathrm{CO} / \mathrm{IR})]=\log \left(1-1.15 \epsilon_{\mathrm{AGN}}\right) .
$$


The solid curves in Figure 14 are from Equation (4) with $\epsilon=50 \%, 65 \%$, and $80 \%$, respectively, and illustrate that Equation (4) can follow the overall distribution of the AGN data points in Figure 14 with some reasonable value of $\epsilon$.

Under the framework presented here, IC 0694 clearly harbors an energetic AGN, perhaps, a heavily dust-enshrouded one, as suggested by some authors (Della Ceca et al. 2002; Alonso-Herrero et al. 2013). This galaxy is not included in Figure 14 because it has $f_{70 \mu \mathrm{m}}\left(30^{\prime \prime}\right)<0.8$ (see Table 4). However, all mid-IR AGN diagnostics point to a low AGN fractional contribution to its bolometric luminosity, at $f_{\text {AGN }} \sim 0.05$ (Díaz-Santos et al. 2017).

Despite the convincing picture of AGN heating of molecular gas unveiled here, we restrict our discussion by pointing out that the sample size of the dominant AGNs presented here is small. As a result, the claims drawn here are in principle still subject to small number statistics.

\subsection{Shocks and Molecular Gas Heating}

NGC 6240 stands out as the only clear outlier in our sample in terms of the mid- $J \mathrm{CO} / \mathrm{IR}$ ratio. The starburst superwinds in NGC 6240 are believed to power large-scale diffuse ionized gas (e.g., Heckman et al. 1987) and shock-excited $\mathrm{H}_{2}$ line emission (e.g., Max et al. 2005). Its SPIRE/FTS CO SLED has been modeled in detail and its excitation was shown to be likely due to shocks (Meijerink et al. 2013). However, there is some difficulty with a stellar shock, which is in turn driven by the SF process, being the main heating mechanism behind the high $\mathrm{CO} / \mathrm{IR}$ ratios observed, because the same SF process should have a far-UV radiation component that is proportional in some way to the mechanical energy of the shock. While the shock itself would heat the gas, the far-UV component would heat the dust which in turn would radiate in the IR. One would therefore expect both the $\mathrm{CO}$ line emission and the IR emission to be impacted by the superwinds and shocks, and therefore that the CO-to-IR luminosity might not be significantly elevated. Indeed, all other well-known superwind galaxies (e.g., M82, Arp 220) show "normal" CO-to-IR-luminosity ratios. In fact, a supernova or stellar wind driven shock has been the most favored gas heating scenario in many recent studies of the mid$J$ CO SLEDs of LIRGs and star-forming galaxies (see Lu et al. 2014 for a list of references). Therefore, shock gas heating does not necessarily raise the $\mathrm{CO} / \mathrm{IR}$ luminosity ratio as long as the shock energy is ultimately derived from SF.

The gas in the nuclear region of NGC 6240 is known to be highly turbulent (Tacconi et al. 1999), with strong molecular gas outflows (Feruglio et al. 2013a, 2013b). Recent ALMA imaging in $\mathrm{HCN}(4-3)$ and $\mathrm{CS}(7-6)$ has shown that the dense gas is concentrated along the ridge between the two nuclei in NGC 6240 (Scoville et al. 2015). ALMA imaging in CO (6-5) would reveal directly whether shocks associated with this nuclear gas component could be responsible for the elevated $\mathrm{CO} / \mathrm{IR}$ ratio.

Objects such as NGC 6240 are likely to be rare, as none of the other galaxies in our sample are like it. Lu et al. (2014) showed two more galaxies from the Herschel archive that resemble NGC 6240 in terms of an elevated CO/IR luminosity ratio. They are NGC 1266 and 3C 293, both of which are known to have significant, non-SF driven shocks such as shocks driven by an AGN-related outflow or radio jet (Ogle et al. 2010; Alatalo et al. 2011; Lanz et al. 2015).

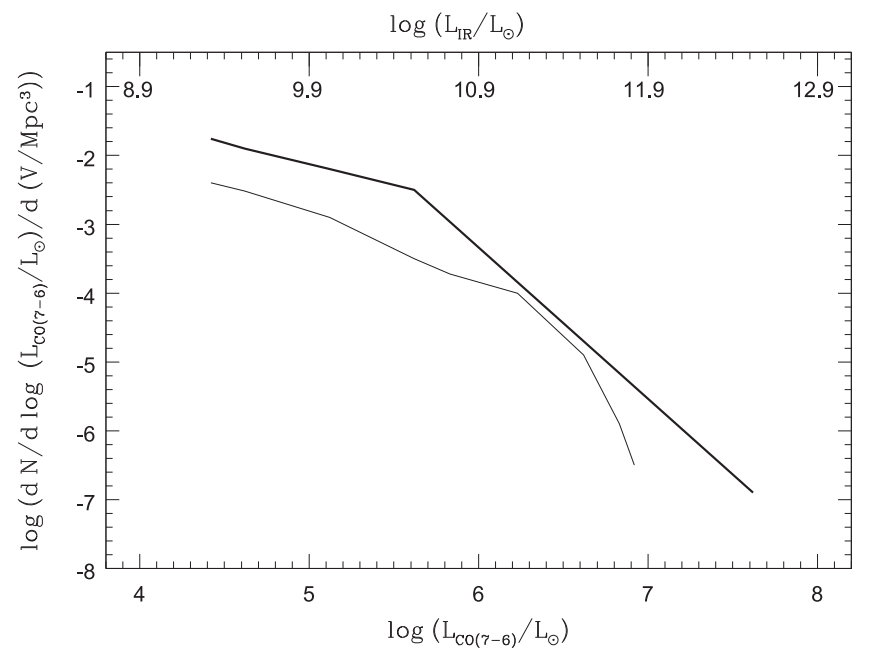

Figure 16. Comparison of our $\mathrm{CO}(7-6) \mathrm{LF}$ scaled from the infrared LF of Sanders et al. (2003) (thick curve) and a local CO (7-6) LF of Lagos et al. (2012) based on a PDR model (thin curve) for $L_{\mathrm{IR}}>2 \times 10^{9} L_{\odot}$, with the logarithmic $L_{\mathrm{IR}}$ scale shown at the top of the plot. The typical uncertainty for our $\mathrm{CO}(7-6) \mathrm{LF}$ is on the order of $0.11 \mathrm{dex}$ (see the text).

\subsection{On Local CO Line Luminosity Functions}

In Section 5.2 we have shown that a mid-J CO line, e.g., $\mathrm{CO}(7-6)$, is potentially a robust tracer of SFR. This opens up the new possibility of characterizing galaxy SFRs at high redshifts by measuring only the flux of $\mathrm{CO}(7-6)$. With a modern facility such as ALMA, this may become routine. The LF of CO (7-6) could therefore develop as a powerful tool to characterize the cosmic evolution of SFR. To this end, one needs to know the present-day $\mathrm{CO}(7-6) \mathrm{LF}$ to serve as the local benchmark. While there are no observational data on such an LF, Lagos et al. (2012) derived a CO (7-6) LF based on a galaxy formation model coupled with a PDR framework for gas heating dominated by far-UV photons.

For most LIRGs, both $L_{\mathrm{IR}}$ and a mid- $J \mathrm{CO}$ line emission trace the same SFR. For lower luminosity galaxies, $L_{\mathrm{IR}}$ may no longer be dominated by young, massive stars. As a result, these two quantities are expected to decouple from each other when $L_{\mathrm{IR}}$ is low enough. Liu et al. (2015) analyzed a large SPIRE/ FTS sample consisting of LIRGs and normal star-forming galaxies and found that $L_{\mathrm{CO}(7-6)}$ and $L_{\mathrm{IR}}$ are coupled nearly linearly down to about $L_{\mathrm{FIR}} \sim 10^{9} L_{\odot}$ (roughly equivalent to $L_{\mathrm{IR}}$ $\approx 2 \times 10^{9} L_{\odot}$ ), with an overall scatter increasing only to $\sim 0.2$ dex at the low-luminosity end. In their study, Liu et al. also applied an aperture correction to $L_{\mathrm{IR}}$ that is similar to $f_{70 \mu \mathrm{m}}\left(35^{\prime \prime}\right)$ defined in this paper. Their result therefore lends support to extending the luminosity limit, above which the $\mathrm{CO}(7-6) / \mathrm{IR}$ ratio remains constant, to $L_{\mathrm{IR}} \sim 2 \times 10^{9} L_{\odot}$.

This constancy of the $\mathrm{CO}(7-6) / \mathrm{IR}$ ratio allows one to derive a local LF of the $\mathrm{CO}(7-6)$ emission from the wellcharacterized local LF of $L_{\mathrm{IR}}$. We show in Figure 16 the $\log$ of such a $\mathrm{CO}(7-6) \mathrm{LF}$ (hereafter referred to as $\left.\mathrm{LF}_{\mathrm{CO}(7-6)}^{\mathrm{IR}-\mathrm{scaled}}\right)$ as a function of $\log L_{\mathrm{CO}(7-6)}$, where $\mathrm{LF}_{\mathrm{CO}(7-6)}^{\mathrm{IR}-\text { scaled }}$ is expressed as $d N / d \log \left(L_{\mathrm{CO}(7-6)} / L_{\odot}\right) / d\left(V / \mathrm{Mpc}^{3}\right)$, with $N$ standing for number of galaxies. This function was scaled from the two-power-law-fitted infrared LF of Sanders et al. (2003) by adopting a constant luminosity scale factor of $\left\langle\log \left(L_{\mathrm{IR}} / L_{\mathrm{CO}(7-6)}\right)\right\rangle=4.88 \pm 0.01$ for (U)LIRGs from $\mathrm{Lu}$ et al. (2015). For comparison we also plotted the model-based $\mathrm{CO}(7-6) \mathrm{LF}$ (hereafter $\mathrm{LF}_{\mathrm{CO}(7-6)}^{\text {model }}$ ) from Lagos et al. (i.e., 
from the solid black curve for $z=0$ in their Figure 5). To have a rough assessment of the uncertainty of $\mathrm{LF}_{\mathrm{CO}(7-6)}^{\mathrm{IR} \text {, }}$, we note that $\log \left(d N / d \log L_{\mathrm{CO}(7-6)}\right)=\log \left(d N / d \log L_{\mathrm{IR}}\right)+\log$ $\left(d \log L_{\mathrm{IR}} / d \log L_{\mathrm{CO}(7-6)}\right)$. The error of the first term on the right side of the above expression can be set to the typical error of 0.08 for the log of the power-law fit of the LF of $L_{\mathrm{IR}}$ in Sanders et al. (see their Table 6). The error of the second term on the right side of the expression can be set to 0.07 , the formal $1 \sigma$ error of $\left\langle\log \left(L_{\mathrm{FIR}} / L_{\mathrm{CO}(7-6)}\right)\right\rangle$ given in the study of Liu et al. The square root of the quadratic sum of these two errors is 0.11, which we take as an estimate on the uncertainty of the $\log$ of $\mathrm{LF}_{\mathrm{CO}(7-6)}^{\mathrm{IR}-\mathrm{scaled}}$.

There are some significant differences between the two curves in Figure 16. First, $\mathrm{LF}_{\mathrm{CO}(7-6)}^{\mathrm{model}}$ from Lagos et al. predicts fewer counts for all luminosity values covered in Figure 16. Second, the turnover of $\operatorname{LF}_{\mathrm{CO}(7-6)}^{\mathrm{IR} \text {-scaled }}$ occurs at $L_{\mathrm{CO}(7-6)} \approx$ $10^{5.6} L_{\odot}$, which is about 0.8 dex lower than the turnover luminosity of $\mathrm{LF}_{\mathrm{CO}(7-6)}^{\text {model }}$. This difference is larger than any that could arise from a systematic underestimate in the SPIRE/FTS $\mathrm{CO}(7-6)$ line fluxes (i.e., as a result of our choice of sinc line profile; see Figure 5). Another difference is that $\mathrm{LF}_{\mathrm{CO}(7-6)}^{\text {model }}$ is much steeper than $\mathrm{LF}_{\mathrm{CO}(7-6)}^{\mathrm{IR}-\text { scaled }}$ at $L_{\mathrm{IR}} \gtrsim 10^{11.5} L_{\odot}$. Both of these differences may reflect the fundamental uncertainty as to whether the far-UV photon gas heating that is the backbone of all PDR models is the main heating mechanism behind the mid$J$ CO line emission in galaxies. For example, recent studies on modeling of the $\mathrm{CO}$ SLEDs of galaxies tend to favor a mechanical heating via shocks (see Lu et al. 2014 for a discussion of this topic). In view of this uncertainty, an $L_{\mathrm{IR}}$-scaled LF such as the one shown in Figure 16 might be a more practical choice for the LF of a mid- $J$ CO line emission, at least for luminous galaxies.

\subsection{Neutral Carbon Line Emission}

The ground state of neutral carbon has a simple three-level, fine-structure system, with the upper and middle energy levels at $62.5 \mathrm{~K}\left({ }^{3} \mathrm{P}_{2}\right)$ and $23.6 \mathrm{~K}\left({ }^{3} \mathrm{P}_{1}\right)$ above the bottom level $\left({ }^{3} \mathrm{P}_{0}\right)$. The ${ }^{3} P_{1} \rightarrow{ }^{3} P_{0}$ transition (i.e., [C I] $\left.609 \mu \mathrm{m}\right)$ has $n_{\mathrm{c}} \approx 470 \mathrm{~cm}^{-3}$ and the ${ }^{3} P_{2} \rightarrow{ }^{3} P_{1}$ transition ([C I] $370 \mu \mathrm{m}$ ) has $n_{\mathrm{c}} \approx 1.2 \times 10^{3} \mathrm{~cm}^{-3}$ (assuming collisions with $\mathrm{H}_{2}$ and a gas temperature of $100 \mathrm{~K}$; see Table 1 in Carilli \& Walter 2013). The latter critical density is similar to that of CO (1 $-0)$. In the optically thin case, the ratio of the two [C I] lines depends only on the excitation temperature $\left(T_{\mathrm{ex}}\right)$ between the energy levels ${ }^{3} \mathrm{P}_{2}$ and ${ }^{3} \mathrm{P}_{1}$. If the gas density is high enough that the neutral carbon ground system is nearly thermalized, the $T_{\mathrm{ex}}$ from the [C I] line ratio should be close to the gas kinetic temperature. In the thermalized case, the observed [C I] fluxes can also be used to infer the total neutral carbon column density. Prior to the advent of Herschel, the [C I] line observations were available for a small number of nearby galaxies (e.g., Büttgenbach et al. 1992; Schilke et al. 1993; Harrison et al. 1995; Stutzki et al. 1997; Gerin \& Phillips 2000; Israel \& Baas 2002; Papadopoulos \& Greve 2004) and for some high-redshift galaxies (see references in Carilli \& Walter 2013).

We plot in Figure 17(a) the line flux ratio of [C I] $370 \mu \mathrm{m}$ to [C I] $609 \mu \mathrm{m}$ as a function of the FIR color for the whole SPIRE/FTS sample, overlaid with implied excitation temperatures $\left(T_{\mathrm{ex}}\right)$ assuming an optically thin case (Stutzki et al. 1997). The relevant excitation temperature range for our LIRGs appears to be from $\sim 15$ and $30 \mathrm{~K}$. This is in agreement with similar findings on some IR active galaxies in the literature (e.g., Weiß et al. 2003). NGC 6240 has the highest $T_{\mathrm{ex}}$ at $40.7 \mathrm{~K}$, among all the sample sources with both [C I] lines detected. Most observations of the $[\mathrm{CI}]$ lines in the literature indicate optically thin cases (e.g., Ojha et al. 2001; Weiß et al. 2003). If this assumption does not hold, the true excitation temperatures would be somewhat higher than those shown in Figure 17(a).

In the classical picture of PDRs (e.g., Kaufman et al. 1999), the [C I] line emission arises from a thin transition layer (of $A_{\mathrm{V}} \sim 1$ to a few) in a gas cloud, between $\mathrm{C}^{+}$and CO. Recent observations have indicated good spatial correspondence between the [C I] $609 \mu \mathrm{m}$ line emission and some low- $J$ rotational transitions from either $\mathrm{CO}$ or ${ }^{13} \mathrm{CO}$ in molecular clouds in our Galaxy (e.g., Ojha et al. 2001; Ikeda et al. 2002; Beuther et al. 2014). This suggests that neutral carbon may be found more ubiquitously throughout a molecular cloud than previously thought, opening up the possibility of using one of the $[\mathrm{C}$ I] lines as an alternative tracer for the mass of molecular gas in distant galaxies (e.g., Papadopoulos \& Greve 2004; Papadopoulos et al. 2004). Figure 17(c) shows that, for our LIRGs, the [C I] $370 \mu \mathrm{m}$ to $\mathrm{CO}(7-6)$ ratio is strongly anticorrelated with $C(60 / 100)$ over the full FIR color range explored here. This ratio drops by at least a factor of 5 when $C$ $(60 / 100)$ increases from 0.4 to 1.2 . In contrast, the [C I] $370 \mu \mathrm{m}$ to $\mathrm{CO}(4-3)$ ratio in Figure 17 (b) has a much weaker overall dependence on $C(60 / 100)$ based on those sources with both [C I] $370 \mu \mathrm{m}$ and $\mathrm{CO}(4-3)$ lines detected, albeit with increased scatter. In general, as $J$ decreases, the ratio of [C I] $370 \mu \mathrm{m}$ (or [C I] $609 \mu \mathrm{m}$ ) to the $\mathrm{CO}$ line of the upper energy level $J$ becomes less dependent on $C(60 / 100)$. This would be evident if one had replaced $\mathrm{CO}(7-6)$ in Figure 17(c) with $\mathrm{CO}(5-4)$ or $\mathrm{CO}(6-5)$. This overall trend can also been seen in our stacked spectra discussed later (see Figure 21 or Table 7). These statistical results suggest that the [C I] line emission in our LIRGs comes predominantly from regions of molecular gas of moderate densities and temperatures, which collectively represent the bulk of the molecular gas mass. It is therefore promising to use the [C I] lines as an alternative molecular gas mass tracer for galaxies at high redshifts.

In Figure 17(c), the dominant AGNs on average show a higher [C I] $370 \mu \mathrm{m}$ to $\mathrm{CO}(7-6)$ ratio than the SF-dominated galaxies at a given $C(60 / 100)$. However, such a systematic difference is not evident in Figure 17(c), which is a plot of the [C I] $370 \mu \mathrm{m}$ line-to-IR luminosity ratio as a function of $C(60 /$ 100). This suggests that the "elevated" [C I] $370 \mu \mathrm{m}$ to $\mathrm{CO}(7$ -6) ratios seen in the dominant AGNs are likely due to the fact that these dominant AGNs have relatively "depressed" CO (7 -6) to IR luminosity ratios, as discussed in Section 5.3.

\subsection{Spectral Stacking and Fainter Lines}

In view of the low detection rates of the fainter spectral lines, we also co-added individual SPIRE/FTS spectra (after registering them all in the rest-frame using a spline interpolation along with the redshift inferred from the observed frequency of the $[\mathrm{N} \mathrm{II}]$ line) to increase sensitivity. Shown in Figures 18-20 (i.e., top panel) are the stacked spectra within three different FIR color bins. These are unweighted median spectra, obtained in the frequency domain, where the instrumental resolution is frequency-independent. Since most of the spectral lines are expected to be unresolved, there is little 


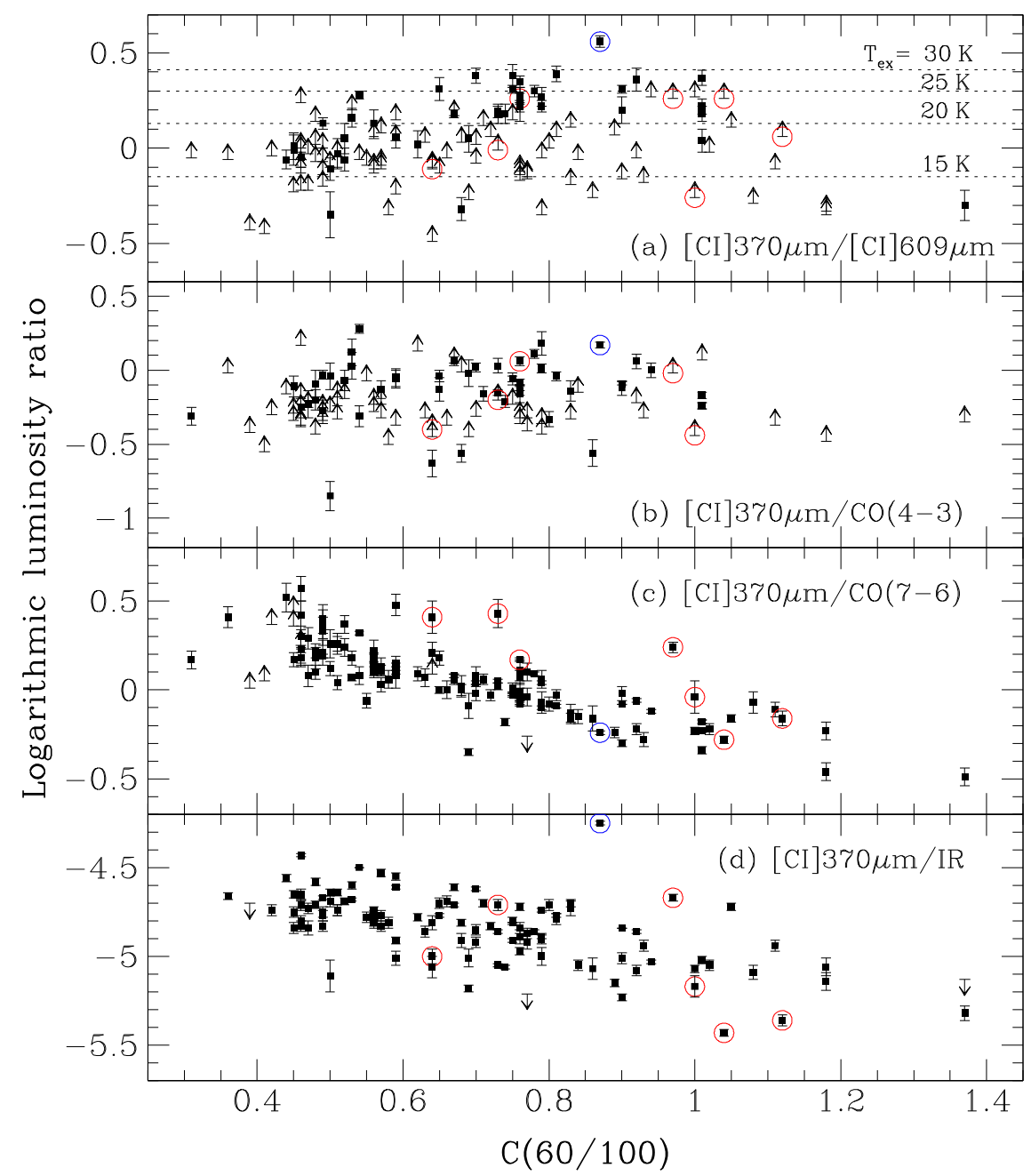

Figure 17. Plots of various [C I] line-related luminosity ratios as a function of the FIR color for our galaxy sample: (a) [C I] $370 \mu \mathrm{m}$ to [C I] $609 \mu \mathrm{m}$, (b) [C I] $370 \mu \mathrm{m}$ to $\mathrm{CO}(4-3)$, (c) [C I] $370 \mu \mathrm{m}$ to $\mathrm{CO}(6-5)$, and (d) [C I] $370 \mu \mathrm{m}$ to the total IR emission. In each plot, arrows indicate the $3 \sigma$ limits when one line involved in the ratio was undetected; the cases where both lines in the ratio were undetected are not included here. NGC 6240 and the known AGNs are further circled in blue and red, respectively. The dotted lines in (a) show the implied gas excitation temperatures from the line ratio using an optically thin case. In (d), only those sources with $f_{70 \mu \mathrm{m}}\left(35^{\prime \prime}\right)>0.8$ are included.

systematic effect from shifting a line in frequency. The weighted median spectra would look similar when the weight used was the inverse of the square of the average noise in SSW or SLW. We prefer the median method over a weighted or unweighted averaging method in order to be less biased to the brightest galaxies in each FIR color bin. We marked the frequency locations of all the main targeted lines and some of the fainter lines in each spectrum, along with the sample standard deviation (middle panel) and the number of individual spectra (bottom panel) used in the stacking process at a given frequency.

In Table 7, we give the peak line flux densities (or the 3- $\sigma$ upper limits), in units of relative Jy, for the $\mathrm{CO}$, [N II], and [C I] lines, as well as a set, of the $\mathrm{H}_{2} \mathrm{O}$ line transitions and $\mathrm{HF}(1-0)$. For an unresolved line, this peak flux density is proportional to its line flux. The error bars at $1 \sigma$ were estimated from the noise in the stacked spectrum, and therefore do not reflect the dispersion (shown in Panel (b) in Figures 18-20) among the individual spectra that went into the stacking process. Our stacking procedure was carried out using the point-source calibrated spectra of individual sources regardless of angular sizes. The frequency-dependent SPIRE/FTS beam could in principle imprint its signature on the relative line fluxes across the frequency range, especially between SSW and SLW, even though the median filtering method we employed should reduce this effect by filtering out those very extended sources that tend to have smaller point-source line fluxes. To check on this systematic effect, we performed the same stacking process on a subset of the sources that are "compact" enough to satisfy $f_{70 \mu \mathrm{m}}\left(17^{\prime \prime}\right)>0.80$ to see if this would result in a significantly different $\mathrm{CO}$ or $\mathrm{H}_{2} \mathrm{O}$ SLED shape. This additional selection process threw out about half of the targets in the FIR cold color bin (i.e., Figure 18), but only a few sources in the FIR warm color bin (Figure 20). We found that the two stacking processes produced similar $\mathrm{CO}$ and $\mathrm{H}_{2} \mathrm{O}$ SLED shapes in each color bin.

Using Table 7, we show in Figure 21 plots of the line intensity, normalized by that of $\mathrm{CO}(6-5)$, as a function of the rest-frame line frequency for the $\mathrm{CO}$ lines (in red), the two [C I] lines (black), the [N II] line (magenta), as well as a suite of $\mathrm{H}_{2} \mathrm{O}$ lines (blue) and $\mathrm{HF}(1-0)$ (green) from the three stacked spectra shown in Figures 18-20, respectively. A detected line is plotted as a filled square whereas an undetected line is shown 
Table 7

Line Intensities from Spectral Stacking Using a Median Method ${ }^{\mathrm{a}}$

\begin{tabular}{|c|c|c|c|c|c|c|c|c|c|c|c|c|c|}
\hline Line & $\begin{array}{c}\text { Freq. } \\
(\mathrm{GHz}) \\
(2)\end{array}$ & $\begin{array}{c}(C(60 / 100) \\
\text { Peak }^{\mathrm{b}}\end{array}$ & $\begin{array}{l}=0.3 \\
\mathrm{rms}^{\mathrm{b}}\end{array}$ & $\begin{array}{c}\text { to } \\
\operatorname{std}^{b}\end{array}$ & $\begin{array}{l}0.6) \\
n_{\mathrm{pts}} \\
(6)\end{array}$ & $\begin{array}{c}(C(60 / 100) \\
\text { Peak }^{\mathrm{b}}\end{array}$ & $\begin{array}{l}=0.6 \\
\mathrm{rms}^{\mathrm{b}}\end{array}$ & $\begin{array}{l}\text { to } \\
\text { std }^{\mathrm{b}}\end{array}$ & $\begin{array}{l}0.9) \\
n_{\mathrm{pts}} \\
(10)\end{array}$ & $\begin{array}{c}(C(60 / 100) \\
\text { Peak }^{\mathrm{b}}\end{array}$ & $\begin{array}{c}=0.9 \\
\mathrm{rms}^{\mathrm{b}} \\
(12)\end{array}$ & $\begin{array}{c}\text { to } \\
\text { std }^{\mathrm{b}} \\
\text { (13) }\end{array}$ & $\begin{array}{l}1.4) \\
n_{\mathrm{pts}} \\
(14)\end{array}$ \\
\hline $\mathrm{CO}(4-3)$ & 461.041 & 1.409 & 0.048 & 1.05 & 47 & 1.503 & 0.042 & 4.97 & 47 & 1.179 & 0.051 & 2.55 & 14 \\
\hline [C I] $609 \mu \mathrm{m}$ & 492.161 & 0.877 & 0.048 & 0.59 & 50 & 0.750 & 0.042 & 2.26 & 52 & 0.559 & 0.051 & 0.70 & 23 \\
\hline $\mathrm{CO}(5-4)$ & 576.268 & 1.399 & 0.048 & 0.87 & 50 & 1.535 & 0.042 & 5.30 & 52 & 1.200 & 0.051 & 2.20 & 23 \\
\hline $\mathrm{CO}(6-5)$ & 691.473 & 1.020 & 0.048 & 0.75 & 50 & 1.424 & 0.042 & 4.91 & 52 & 1.576 & 0.051 & 2.16 & 23 \\
\hline $\mathrm{H}_{2} \mathrm{O}\left(2_{11}-2_{02}\right)$ & 752.033 & $<0.144$ & 0.048 & 0.24 & 50 & 0.192 & 0.042 & 0.65 & 52 & 0.243 & 0.051 & 1.25 & 23 \\
\hline $\mathrm{CO}(7-6)$ & 806.652 & 0.800 & 0.048 & 0.72 & 50 & 1.428 & 0.042 & 4.75 & 52 & 1.535 & 0.051 & 2.47 & 23 \\
\hline [C I] $370 \mu \mathrm{m}$ & 809.342 & 1.353 & 0.048 & 0.86 & 50 & 1.389 & 0.042 & 5.02 & 52 & 0.971 & 0.051 & 1.57 & 23 \\
\hline $\mathrm{CO}(8-7)$ & 921.800 & 0.634 & 0.048 & 0.70 & 50 & 1.021 & 0.042 & 4.33 & 52 & 1.625 & 0.051 & 2.65 & 23 \\
\hline $\mathrm{H}_{2} \mathrm{O}\left(2_{02^{-}} 1_{11}\right)^{c}$ & 987.927 & 0.347 & 0.048 & 0.58 & 50 & 0.391 & 0.042 & 1.00 & 52 & 0.489 & 0.051 & 1.72 & 23 \\
\hline $\mathrm{CO}(9-8)$ & 1036.912 & 0.407 & 0.051 & 0.50 & 50 & 0.991 & 0.046 & 2.76 & 52 & 1.257 & 0.049 & 2.07 & 23 \\
\hline $\mathrm{H}_{2} \mathrm{O}\left(3_{12}-3_{03}\right)$ & 1097.365 & $<0.153$ & 0.051 & 0.28 & 50 & 0.191 & 0.046 & 0.77 & 52 & 0.413 & 0.049 & 1.45 & 23 \\
\hline $\mathrm{H}_{2} \mathrm{O}\left(1_{11}-0_{00}\right)$ & 1113.343 & $<0.153$ & 0.051 & 0.35 & 50 & $<0.138$ & 0.046 & 0.51 & 52 & $<0.147$ & 0.049 & 1.65 & 23 \\
\hline $\mathrm{CO}(10-9)$ & 1151.985 & 0.366 & 0.051 & 0.36 & 50 & 0.611 & 0.046 & 2.37 & 52 & 1.417 & 0.049 & 1.84 & 23 \\
\hline $\mathrm{H}_{2} \mathrm{O}\left(3_{21}-3_{12}\right)$ & 1162.912 & $<0.153$ & 0.051 & 0.35 & 50 & 0.301 & 0.046 & 1.22 & 52 & 0.550 & 0.049 & 2.78 & 23 \\
\hline $\mathrm{H}_{2} \mathrm{O}\left(4_{22}-4_{13}\right)$ & 1207.639 & $<0.153$ & 0.051 & 0.30 & 50 & $<0.138$ & 0.046 & 0.51 & 52 & 0.278 & 0.049 & 1.02 & 23 \\
\hline $\mathrm{H}_{2} \mathrm{O}\left(2_{20}-2_{11}\right)$ & 1228.789 & 0.171 & 0.051 & 0.40 & 50 & 0.265 & 0.046 & 0.88 & 52 & 0.250 & 0.049 & 1.34 & 23 \\
\hline HF (1-0) & 1232.476 & -0.208 & 0.051 & 0.50 & 50 & $<0.138$ & 0.046 & 0.61 & 52 & -0.163 & 0.049 & 1.87 & 23 \\
\hline CO (11-10) & 1267.014 & 0.199 & 0.051 & 0.44 & 50 & 0.597 & 0.046 & 2.05 & 52 & 0.913 & 0.049 & 1.56 & 23 \\
\hline CO (12-11) & 1381.995 & $<0.153$ & 0.051 & 0.33 & 50 & 0.387 & 0.046 & 1.54 & 52 & 0.880 & 0.049 & 1.22 & 23 \\
\hline $\mathrm{H}_{2} \mathrm{O}\left(5_{23}-5_{14}\right)$ & 1410.618 & $<0.153$ & 0.051 & 0.32 & 50 & $<0.138$ & 0.046 & 0.42 & 52 & $<0.147$ & 0.049 & 0.66 & 23 \\
\hline$[\mathrm{N} \mathrm{III}] 205 \mu \mathrm{m}$ & 1461.134 & 5.840 & 0.051 & 3.42 & 50 & 4.563 & 0.046 & 12.90 & 52 & 1.568 & 0.049 & 2.89 & 23 \\
\hline CO (13-12) & 1496.923 & $<0.153$ & 0.051 & 0.36 & 50 & 0.253 & 0.046 & 1.05 & 52 & 0.688 & 0.049 & 1.26 & 23 \\
\hline
\end{tabular}

Notes.

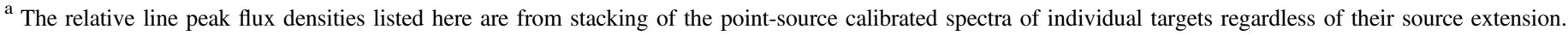

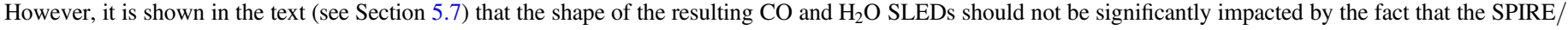
FTS beam is frequency-dependent.

b These are respectively the line peak flux density, the rms noise in continuum, and the sample standard deviation, all in units of relative Jy.

c The line flux of $\mathrm{H}_{2} \mathrm{O}\left(2_{02}-1_{11}\right)$ was measured in the SLW spectral segment.

either as an upper limit if it is a $\mathrm{CO}$ line or as a range of $\pm 3 \sigma$ if it is a $\mathrm{H}_{2} \mathrm{O}$ line or $\mathrm{HF}(1-0)$. The $\mathrm{CO}(4-3)$ and $\mathrm{CO}(14-13)$ lines are excluded here as both of them were stacked over fewer individual spectra. Note that the [N II] line is not shown in (a) or (b), as the line is too bright to fit within the plot. As a comparison, we also plot, in each color bin, the CO SLED (in red crosses) from the stacking of the subset of targets with $f_{70 \mu \mathrm{m}}\left(17^{\prime \prime}\right)>0.80$. For clarity, the crosses were manually offset in frequency by $-10 \mathrm{GHz}$. There appears to be no significant difference between the two sets of CO SLEDs shown here, especially in (a) and (b). The difference is apparently larger for the warm FIR color bin in (c), but this is largely due to the fact that the intrinsic scatter in individual $\mathrm{CO}$ SLED shape is large in the first place (see Figure 11). The same conclusion could have been drawn if we had done a similar comparison on the $\mathrm{H}_{2} \mathrm{O}$ SLEDs here. As a result, the relative $\mathrm{CO}$ and $\mathrm{H}_{2} \mathrm{O}$ line fluxes in Table 7 are not significantly affected by the fact that the SPIRE/FTS beam varies significantly across SSW and SLW.

The CO SLEDs from the stacked spectra in Figure 21 are consistent with their corresponding median CO SLEDs based on the brightest sample galaxies (see Figure 11 and Table 6). This confirms that the average CO SLED shape is indeed correlated primarily with the FIR color, not significantly influenced by apparent flux or luminosity. Also note in Figure 21 that, while the relative flux strength between [C I] $370 \mu \mathrm{m}$ and $\mathrm{CO}(7-6)$ varies greatly across the 3 FIR colors, that between [C I] $370 \mu \mathrm{m}$ and $\mathrm{CO}$ (5-4) varies much less so. This reinforces our earlier conclusion that the ratio of [C I] $370 \mu \mathrm{m}$ to a CO line of the upper energy level $J$ becomes less dependent on $C(60 / 100)$ as $J$ decreases (see Figures 17(b) and (c)). As a further application of the stacked spectra, we use Figure 21 to study below how the relative strengths of $\mathrm{H}_{2} \mathrm{O}$ lines vary as FIR color increases.

\subsection{1. $\mathrm{H}_{2} \mathrm{O}$ Vapor Lines}

While $\mathrm{H}_{2} \mathrm{O}$ is an abundant molecular species in the ISM, it remains mostly as ice on dust grains (e.g., van Dishoeck et al. 2011). Only in warm molecular gas does it exist in vapor form and can be detected in terms of its rotational transitions in emission or absorption depending on the background continuum. These lines were detected by the Infrared Space Observatory in a small number of galaxies, including Arp 220 (González-Alfonso et al. 2004), NGC 253, and NGC 1068 (Goicoechea et al. 2005), and Mrk 231 (González-Alfonso et al. 2008). With the improved sensitivity of Herschel, the sample of galaxies with $\mathrm{H}_{2} \mathrm{O}$ line detections has been expanded to include additional bright galaxies and (U)LIRGs (e.g., González-Alfonso et al. 2010, 2012, 2013; Van der Werf et al. 2010; Weiß et al. 2010; Rangwala et al. 2011; Kamenetzky et al. 2012; Spinoglio et al. 2012; Appleton et al. 2013). Yang et al. (2013) collected a sample of 176 galaxies with either published or unpublished SPIRE/FTS spectra and examined simple flux-flux correlations between the luminosity of a water 


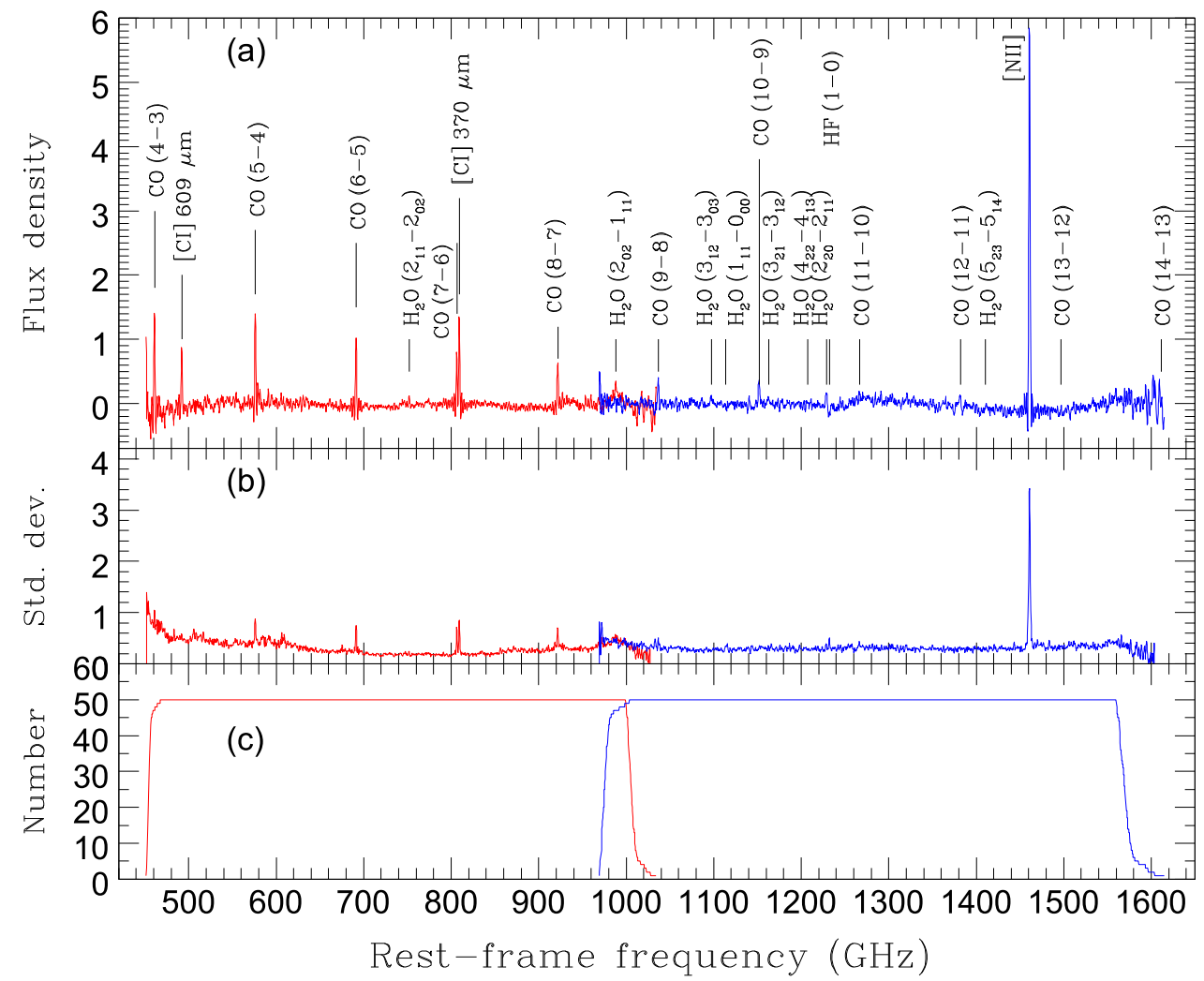

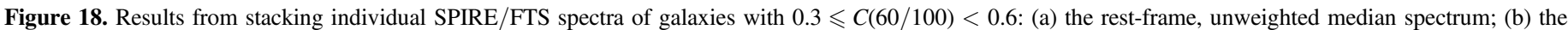

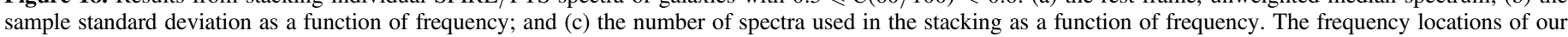
main targeted lines, as well as a number of $\mathrm{H}_{2} \mathrm{O}$ lines and $\mathrm{HF}(1-0)$, are marked in (a).

line and $L_{\mathrm{IR}}$ for a subset of 45 galaxies with at least one water line detected. They found that, in general, the water line luminosity scales nearly linearly with $L_{\mathrm{IR}}$ and favored the IR pumping plus collisional excitation model proposed by González-Alfonso et al. (2010). However, if the water lines from highly excited energy levels in (U)LIRGs are dominated by IR photon pumping, one would expect the shape of the SLED of the $\mathrm{H}_{2} \mathrm{O}$ line emission to be highly sensitive to the FIR color or dust temperature $T_{\text {dust }}$ (or IR photon density) (González-Alfonso et al. 2014a). However, such a trend was not observed by Yang et al. (2013). We investigate this subject further here.

Since the $\mathrm{H}_{2} \mathrm{O}$ lines were only detected in a fraction of our sample galaxies, we chose to make use of the data in Table 7 from the stacked spectra. As shown in Figure 21, only two individual $\mathrm{H}_{2} \mathrm{O}$ lines, $\mathrm{H}_{2} \mathrm{O}\left(2_{02}-1_{11}\right)$ and $\mathrm{H}_{2} \mathrm{O}\left(2_{20}-2_{11}\right)$, are detected in all three stacked spectra. Both these lines show only small variations in their $\mathrm{CO}(6-5)$-normalized line flues: 0.28 to 0.34 for $\mathrm{H}_{2} \mathrm{O}\left(2_{02}-1_{11}\right)$ and 0.16 to 0.19 for $\mathrm{H}_{2} \mathrm{O}\left(2_{20}-2_{02}\right)$. These two lines are characterized by their relatively low upper energy levels of $E_{\text {up }}=100-200 \mathrm{~K}$. In contrast, such variations are much larger for water lines with higher $E_{\mathrm{up}}$. For example, the $\mathrm{CO}(6-5)$-normalized flux of $\mathrm{H}_{2} \mathrm{O}\left(3_{21}-3_{12}\right)$ (with $E_{\text {up }}=305 \mathrm{~K}$ ) increases from a value less than 0.15 in the FIR cold subsample to a value of 0.35 in the FIR warm subsample. Since the $\mathrm{CO}(6-5)$ line luminosity traces the total SFR well, this quantitative observation suggests that, as $C(60 /$ 100) increases, only $\mathrm{H}_{2} \mathrm{O}$ emission lines associated with a highenough $E_{\text {up }}$ are enhanced above the average line luminosity per SFR.
We explore the above phenomenon further in Figure 22, where we show the resulting $\mathrm{H}_{2} \mathrm{O}$ SLEDs from each of the three stacked spectra by plotting the strength of a $\mathrm{H}_{2} \mathrm{O}$ line, relative to that of $\mathrm{H}_{2} \mathrm{O}\left(2_{02}-1_{11}\right)$, as a function of the upper level energy of that line. The $\mathrm{H}_{2} \mathrm{O}$ SLEDs of the 3 FIR color bins are differentiated by different colors. (One can connect the data points of the same color to see more clearly the differences between the individual SLEDs.) It is evident that the $\mathrm{H}_{2} \mathrm{O}$ SLED of the warmest FIR color bin of $0.9 \leqslant C(60 / 100)<1.4$ (shown in blue) is "tilted" more toward the high- $E_{\text {up }}$ lines than the $\mathrm{H}_{2} \mathrm{O}$ SLED of the least warm FIR color bin of $0.3 \leqslant C(60 /$ $100)<0.6$ (shown in red). In other words, as the FIR color increases, the strengths of the water lines with $E_{\text {up }}>200 \mathrm{~K}$ are increasingly enhanced relative to those of the water lines with $E_{\text {up }} \lesssim 200 \mathrm{~K}$. The clearest difference among the different FIR color bins is seen in $\mathrm{H}_{2} \mathrm{O}\left(3_{21}-3_{12}\right)$ with $E_{\text {up }} \sim 300 \mathrm{~K}$, where the vertical displacement between the successive FIR color bins has a significance of at least 2.5 times the uncertainty inferred from the error bars shown. However, the most significant point is that the observed pattern in Figure 22 is consistent with the IR pumping model predictions by González-Alfonso et al. (2014a), who showed that an increasing $T_{\text {dust }}$ enhances the relative strengths of the $\mathrm{H}_{2} \mathrm{O}$ lines of $E_{\text {up }}>200 \mathrm{~K}$ (see their Figure 3).

The $\mathrm{H}_{2} \mathrm{O}$ line of the lowest $E_{\text {up }}$ is $\mathrm{H}_{2} \mathrm{O}\left(1_{11}-0_{00}\right)$ at 1113.3 GHz. This line is in absorption in more than half of the detections, with the strong absorption case in Arp 220 (see Figure 2) being a good example. In contrast, the $\mathrm{H}_{2} \mathrm{O}$ lines of the highest $E_{\text {up }}$ are almost always in emission in our sample, e.g., $\mathrm{H}_{2} \mathrm{O}\left(3_{21}-3_{12}\right)$ and $\mathrm{H}_{2} \mathrm{O}\left(5_{23}-5_{14}\right)$. This contrast supports 


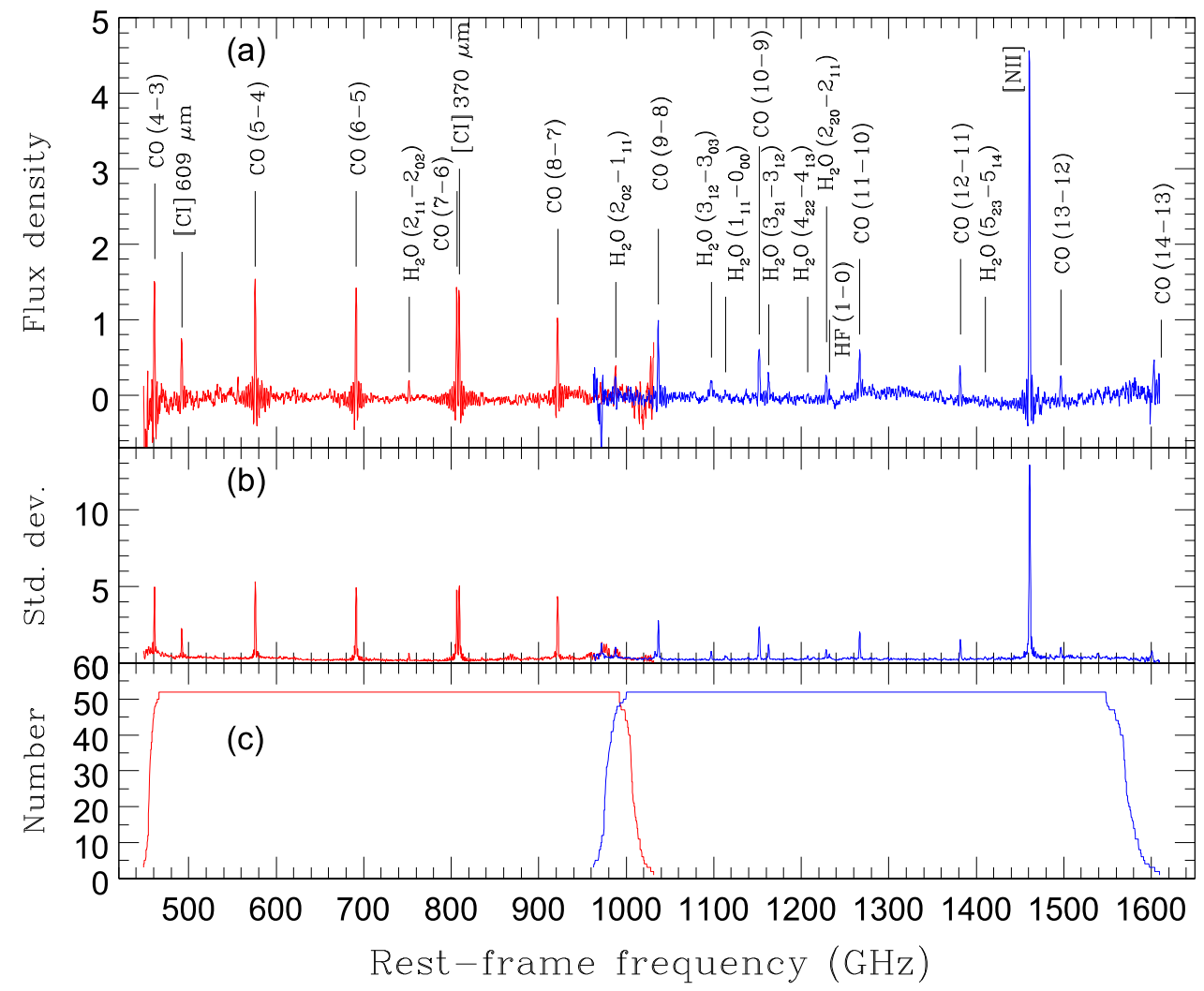

Figure 19. Same as Figure 18, but using the sample galaxies with $0.6 \leqslant C(60 / 100)<0.9$.

the picture that lower-energy water lines are overall not significantly affected by the IR pumping.

\subsection{2. $H F(1-0)$}

Despite its relatively low abundance in the ISM, Fluorine (F) can react with molecular hydrogen $\left(\mathrm{H}_{2}\right)$ to form $\mathrm{HF}$, which locks up most $\mathrm{F}$ atoms. If the $\mathrm{F} / \mathrm{H}$ abundance ratio is more or less fixed, the formation process of $\mathrm{HF}$ involving $\mathrm{H}_{2}$ implies that its number density may scale with that of $\mathrm{H}_{2}$, thus can serve as an alternative tracer of the total molecular mass (Neufeld et al. 2005). The lowest rotational transition, $\mathrm{HF}(1-0)$, has a frequency of $1232.476 \mathrm{GHz}$, which is within the SPIRE/FTS frequency coverage. The critical density for collisional excitation of this transition with $\mathrm{H}_{2}$ is quite high, at about $5 \times 10^{10} \mathrm{~cm}^{-3}$ (Neufeld et al. 2005). As a result, the line is typically observed in absorption in the Milky Way (e.g., Neufeld et al. 2010; Phillips et al. 2010; Sonnentrucker et al. 2010, 2015; Monje et al. 2011). With the assumption that most $\mathrm{HF}$ is in its ground rotational state, the observed absorption line strength of HF $(1-0)$ has been used to infer the column density of HF along the line of sight, and thus, indirectly, that of $\mathrm{H}_{2}$ (e.g., Neufeld et al. 2005; Monje et al. 2011, 2014). Recent Herschel observations of Mrk 231 (Van der Werf et al. 2010), NGC 7130 (Pereira-Santaella et al. 2013), and the Orion Bar in our own Galaxy (Van der Tak et al. 2012) have revealed that this line could be predominantly in emission. Possible excitation mechanisms for the emission have been discussed in the literature, including near-infrared radiative pumping, chemical pumping, and collision with electrons (Van der Tak et al. 2012; PereiraSantaella et al. 2013), but with no consistent picture emerging at this point. The observational fact that HF (1 $-0)$ can be either in emission or absorption at a galactic level suggests that it might be not straightforward to use the observed flux of this line to infer the total molecular gas content of a galaxy.

We can look to see whether there are any trends in the ratio of the HF $(1-0)$ flux to $F_{\mathrm{IR}}$ or the underlying continuum for our galaxy sample. To this end, we used a continuum flux based on the more recent HIPE 14 flux calibration. While the line flux calibration improved very marginally since HIPE 11, the continuum flux calibration has improved significantly. We further estimated the telescope residual continuum signal by fitting a polynomial of the order of 5 to the median spectrum of the surrounding detectors (i.e., for SLW, these are the five detectors in the first detector ring; for SSW, these are the five detectors in the second detector ring that also co-align spatially with the aforementioned five SLW detectors; see Swinyard et al. 2014). We can calculate the value of this polynomial function at any given frequency. For our galaxy sample, the mean value is about 0.1 Jy over the SSW frequency range, with a sample standard deviation of $\sim 0.2 \mathrm{Jy}$. (This residual telescope signal is much less in SLW.) This polynomial fit of the telescope residual continuum was then subtracted from the target spectrum extracted from the central detectors before any continuum flux was measured. We then measured the median continuum flux densities in two line-free frequency ranges: $1169-1221 \mathrm{GHz}$ and $1273-1305 \mathrm{GHz}$ (in the rest-frame), which bracket the HF line in frequency. The continuum flux density at the frequency of the HF line, derived as a linear interpolation of these two flux densities, was used to calculate the line EW. 


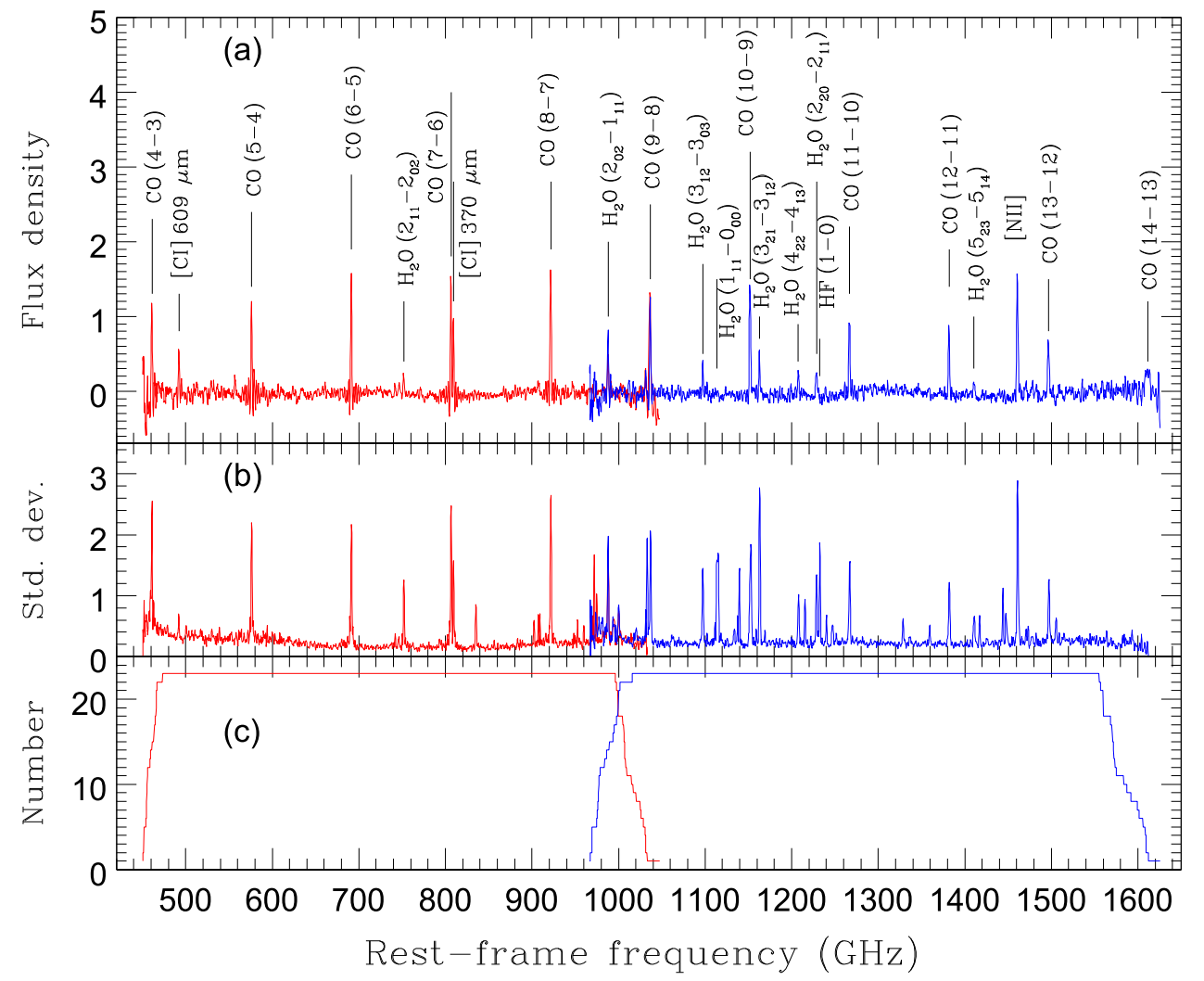

Figure 20. Same as Figure 19, but using the sample galaxies with $0.9 \leqslant C(60 / 100)<1.4$.

If the HF (1-0) line was detected for a galaxy, its integrated line flux was calculated using a sinc line profile and tabulated in Table 5, where a negative flux denotes a line in absorption; in the case of a non-detection we derived a $3 \sigma$ upper limit for the flux amplitude using the local noise level in the spectrum. In Figure 23 we plot, as a function of the FIR color, (a) the ratio of the absolute HF (1-0) luminosity to $L_{\mathrm{IR}}$ and (b) the EW of the absolute HF $(1-0)$ line flux or its $3 \sigma$ upper limit for our sample galaxies. For a detected line, we use different colors to separate an emission case (in red) from an absorption case (in blue). The dominant AGNs as defined in Section 2.2 are further circled in magenta. In either plot, there is no clear segregation trend between the emission and absorption cases as $C(60 / 100)$ increases, nor any significant difference between the strong AGNs and those dominated by SF. The emission and absorption cases appear to be roughly equally represented in our sample so that $\mathrm{HF}(1-0)$ is either undetected or only marginally detected in our stacked spectra (see Figure 21). One conjecture is that the observed $\mathrm{HF}(1-0)$ line is a net of individual emission and absorption sites along the line of sight and could be in either emission or absorption depending on whether the emission sites collectively outnumber the absorption sites. Without a good understanding of the physical picture behind the integrated $\mathrm{HF}(1-0)$ line flux, it is rather uncertain to use the observed HF line flux to infer the molecular gas mass of a galaxy, especially at high redshift where the spatial resolution is usually poor.

There could be possibly a weak trend for a lower absolute $\mathrm{HF}(1-0)$ luminosity to $L_{\mathrm{IR}}$ ratio on average at a higher $C(60 /$ 100) in Figure 23(a). However, the fact the emission and absorption cases of HF (1-0) are well mixed in Figure 23(a) makes it challenging to draw any meaningful inference from such a trend. Figure 23(a) shows that, of all the emission cases we detected, the $L_{\mathrm{HF}} / L_{\mathrm{IR}}$ is the largest $\left(\approx 1.3 \times 10^{-5}\right)$ in the case of IRAS $05442+1732$. On the other hand, the strongest absorption case in Figure 23(b) is NGC 0023, which has an EW of $106( \pm 16) \mathrm{km} \mathrm{s}^{-1}$.

\section{Summary}

In this paper we presented a Herschel SPIRE 194-671 $\mu \mathrm{m}$ spectroscopic survey of 121 galaxies belonging to a complete, flux-limited sample of 123 LIRGs down to a total IR flux of $6.5 \times 10^{-13} \mathrm{~W} \mathrm{~m}^{-2}$, selected from the GOALS. This program complements the other two Herschel surveys on the GOALS sample: a broadband photometric survey at 70, 100, 160, 250, 350 , and $500 \mu \mathrm{m}$ (J. Chu et al. 2017, in preparation) and a spectroscopic line survey of some major FIR fine-structure lines (Díaz-Santos et al. 2013).

From the SPIRE spectra presented here, we derived and tabulated the integrated line fluxes or upper limits for (a) the CO rotational transitions of $J$ to $J-1$ over $4 \leqslant J \leqslant 13$, being most complete for the $\mathrm{CO}$ lines in the mid- $J$ regime (i.e., $5 \leqslant J \leqslant 10$ ), (b) the fine-structure [N II] line at $205 \mu \mathrm{m}$, with a detection completeness at nearly $100 \%$, and (c) the two finestructure lines of the neutral carbon in its ground state, [C I] at 609 and $370 \mu \mathrm{m}$, with a very high detection completeness for the $370 \mu \mathrm{m}$ line. We also tabulated additional (usually fainter) spectral lines detected in many individual targets, such as some of the rotational transitions of $\mathrm{H}_{2} \mathrm{O}$ vapor and $\mathrm{HF}(1-0)$, the $J=1$ to 0 rotational transition of hydrogen fluoride. The [N II] data presented here have been statistically analyzed in detail in Zhao et al. (2013, 2016a). 


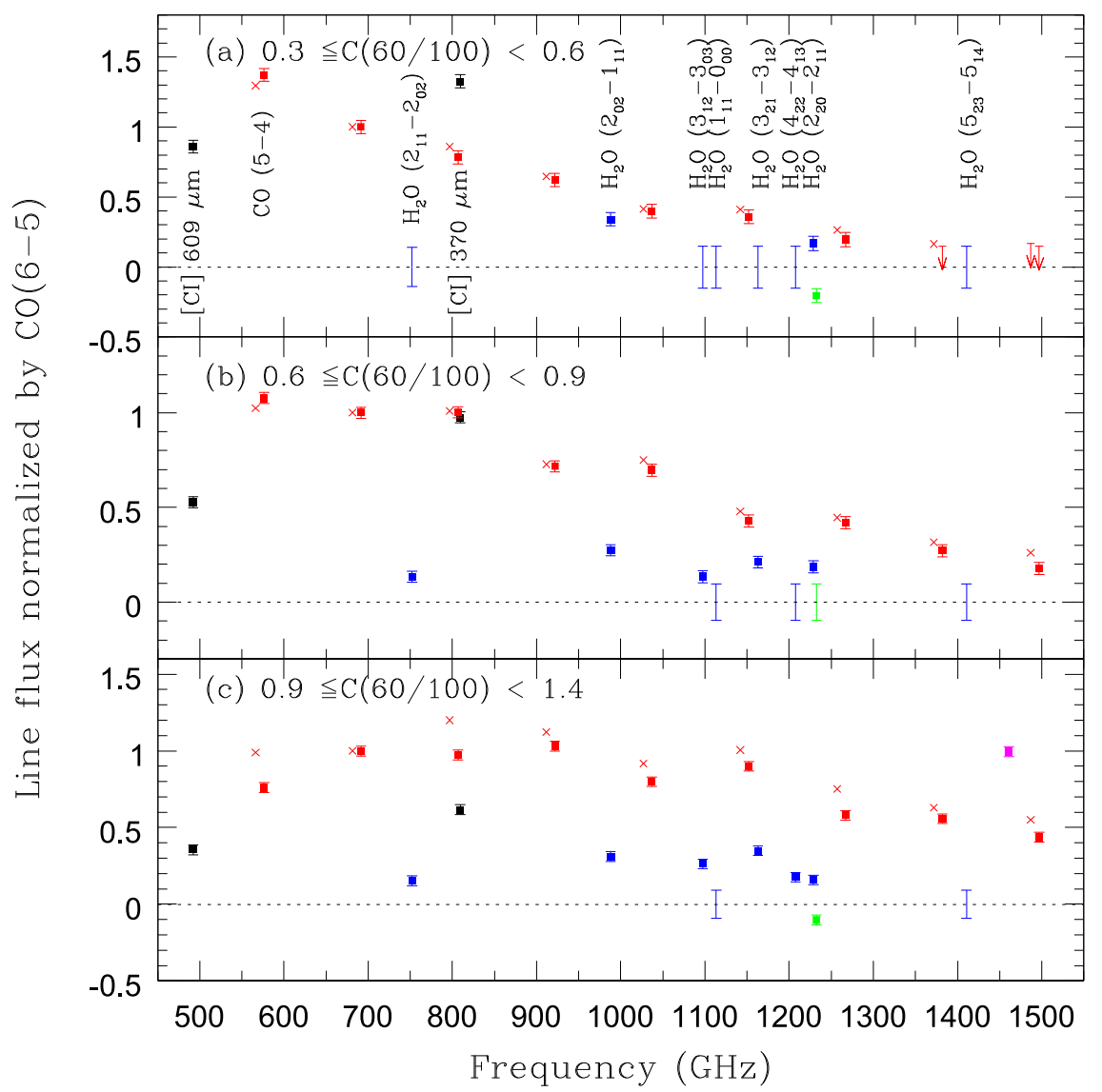

Figure 21. Panels (a) to (c) are plots of the line intensity or its upper limit, normalized by the intensity of $\mathrm{CO}(6-5)$, as a function of the line frequency, for the $\mathrm{CO}$ lines (in red), the two [C I] lines (black), the [N II] line (magenta), as well as a suite of $\mathrm{H}_{2} \mathrm{O}$ lines (blue) and $\mathrm{HF}(1-0)$ (green) from the three stacked spectra shown in Figures 18-20, respectively. The normalized [N II] line fluxes are 5.72, 3.20, and 1.00 in (a), (b) and (c), respectively. Therefore this line is off the scale in both (a) and (b). Detected lines are shown as filled squares. For an undetected $\mathrm{CO}$ line, its $3 \sigma$ upper limit is plotted; for an undetected $\mathrm{H}_{2} \mathrm{O}$ or $\mathrm{HF}(1-0)$ line, its $\pm 3 \sigma$ range is shown to enclose both emission and absorption possibilities. Some of the lines are labeled in (a) to guide line identifications. For the CO lines, we also show the results (in red crosses) from a similar stacking procedure, but there results are limited to the subset of "compact" targets with $f_{70 \mu \mathrm{m}}\left(17^{\prime \prime}\right)>0.80$. For clarity, this second CO data set is offset by $-10 \mathrm{GHz}$ along the frequency axis in each plot.

We found that the overall shape of a CO SLED over $4 \leqslant J \leqslant 13$ is much better correlated with FIR color than with IR luminosity $L_{\mathrm{IR}}$. From this we inferred that the intensity of the dust heating radiation field is the main determinant of the overall excitation temperature of dense molecular gas in these galaxies. The CO-line-to-IR-luminosity ratios presented here confirm our earlier analysis ( $\mathrm{Lu}$ et al. 2014), calling for a minimum of two distinct galactic molecular gas components: (i) a cold component, which emits the $\mathrm{CO}$ lines primarily at $J \lesssim 4$ and likely represents the same gas phase as traced by $\mathrm{CO}(1-0)$, and (ii) a warm and dense gas component, dominant over the mid- $J$ regime $(4<J \lesssim 10)$, which is intimately related to current SF.

Based on a quantitative estimate of the fractional contribution of AGN to the bolometric luminosity, $f_{\mathrm{AGN}}$, we singled out a set of seven dominant AGNs with $f_{\mathrm{AGN}}>50 \%$. The mid- $J$ CO SLEDs of these dominant AGNs are statistically identical to those of the galaxies dominated by SF, but on average have a lower mid-J CO-to-IR-luminosity ratio. Considering the galaxy sample as a whole (excluding NGC 6240 ), we observe an overall trend toward a falling mid- $J$ COto-IR-luminosity ratio with increasing $f_{\mathrm{AGN}}$. Combining our SPIRE/FTS data with CO data of $J>13$ from the literature, we further illustrated that the lower mid-J CO-to-IR ratios of these dominant AGNs are likely a result of an excess of $\mathrm{CO}$ emission in the $J>10 \mathrm{CO}$ lines, likely associated with AGN heating of molecular gas. As a result, the mid- $J$ CO line emission in a LIRG is predominantly powered by SF, irrespective of whether the galaxy harbors an energetic AGN or not.

NGC 6240 is a clear outlier in our sample with a mid- $J$ COto-IR luminosity ratio much higher than that of any other galaxy in the sample. We argued that the likely gas heating scenario in NGC 6240 involves shocks unrelated to current SF.

The relatively tight correlation between the mid- $J$ CO line emission and the total IR emission for our LIRGs implies that the shape of the local LF of a mid-J CO line should be close to that of $L_{\mathrm{IR}}$ except for a constant scale factor in luminosity. The $\mathrm{CO}(7-6) \mathrm{LF}$ determined this way differs significantly from a PDR-model-based LF, suggesting either that the PDR heating mechanism may need to be revised or that it may not be the dominant heating mechanism for this warm molecular gas phase.

The ratios of the two neutral carbon lines imply a relatively modest excitation temperature, ranging from 15 to $30 \mathrm{~K}$. This, together with the fact that the [C I] line flux scales more linearly with $\mathrm{CO}(4-3)$ than with a higher- $J \mathrm{CO}$ line (e.g., $\mathrm{CO}(7-6)$ ), suggests that the $[\mathrm{C} \mathrm{I}]$ line emission is physically more related to the cold $\mathrm{CO}$ component (i) defined above. 


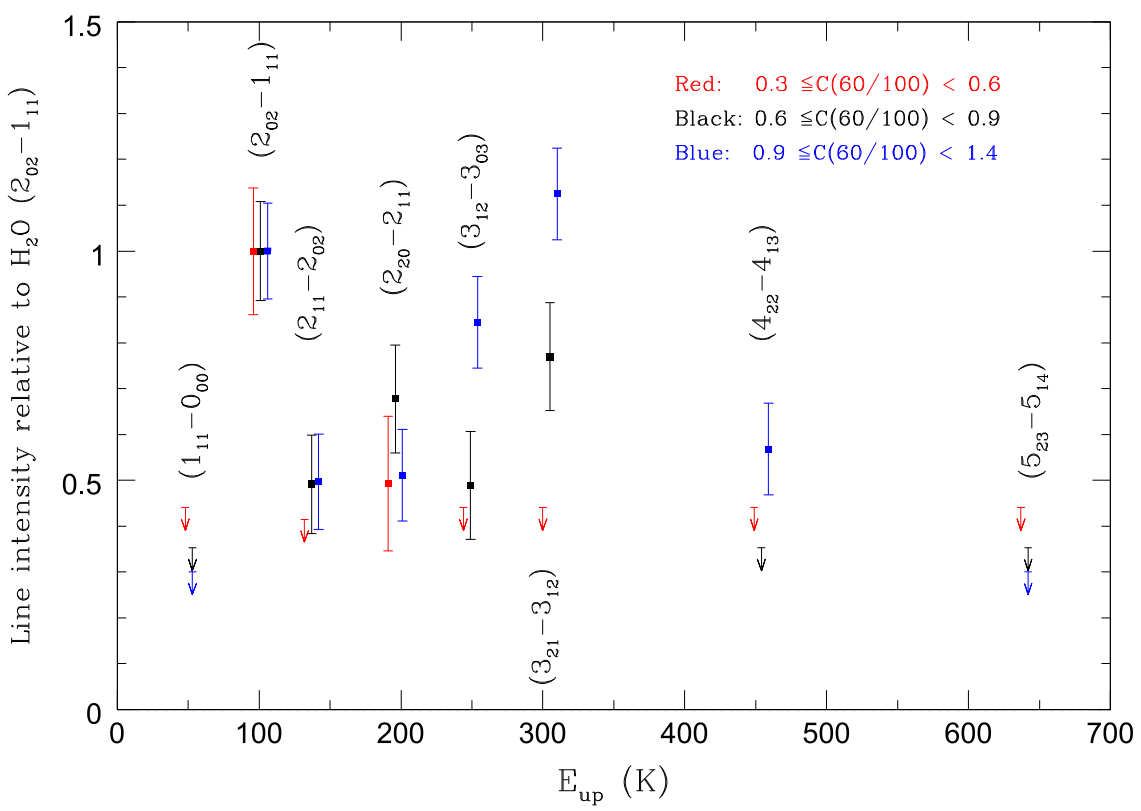

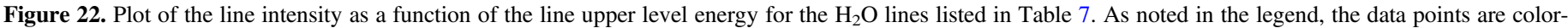

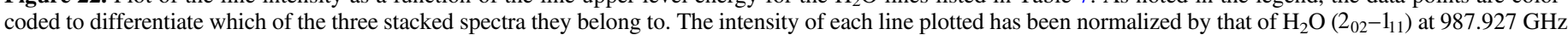

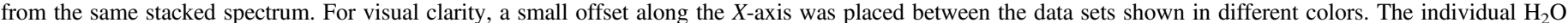
transitions are labeled in the plot. Note that the $3 \sigma$ upper limit shown for a non-detection also constrains the amplitude of the subject line if it is in absorption.

To better measure some of the fainter lines in the SPIRE spectra, we derived stacked spectra of three subsamples of different FIR colors. Our results indicate an evolution of the $\mathrm{H}_{2} \mathrm{O}$ SLED as the FIR color becomes warmer in a direction that is consistent with an IR photon pumping framework suggested by González-Alfonso et al. (2014a).

The HF (1-0) line was detected in emission in some sources, but in absorption in others. No correlation with the FIR color was identified. This, together with the fact that $\mathrm{HF}(1$ $-0)$ is only barely detected in our stacked spectra, suggests that both emission and absorption may be present in any given galaxy. As a result, it is not straightforward to use the observed $\mathrm{HF}(1-0)$ line strength (in either absorption or emission) to estimate the total molecular gas mass in a galaxy.

This paper benefited from a number of thoughtful comments made by the anonymous referee. The work presented here is based in part on observations made with Herschel, a European Space Agency Cornerstone Mission with significant participation by NASA. Support for this work was provided in part by NASA through an award issued by JPL/Caltech. N.L. acknowledges partial support from the Natural Science Foundation of China (NSFC) under grant No. 11673028. Y.Z. is partially supported by NSFC grant No. 11673057. Y.G. and Y.Z. are partially supported by NSFC grants No. 11173059, 11390373, and 11420101002, and the CAS pilot-b project No. XDB09000000. T.D.-S. acknowledges support from ALMA-CONICYT project 31130005 and FONDECYT 1151239. V.C. acknowledges partial support from the EU FP7 Grant PIRSES-GA-2012316788. K.I. acknowledges support by the Spanish MINECO under grantAYA2013-47447-C3-2-P and MDM-2014-0369 of ICCUB (Unidad deExcelencia "María de Maeztu"). G.C.P. was supported by a FONDECYT postdoctoral fellowship (No. 3150361). This research has made use of the NASA/ IPAC Extragalactic Database (NED), which is operated by the Jet Propulsion Laboratory, California Institute of Technology, under contract with the National Aeronautics and Space Administration.

\section{Appendix \\ Notes on Infrared Luminosities of Targets with Companions}

The targets in Table 1 with their $L_{\mathrm{IR}}$ value marked by "(*)" are all in a (either physical or projected) system of multiple galaxies based on optical and near-IR imaging data (see Howell et al. 2010; and for an updated work on this, see J. M. Mazzarella et al. 2017, in preparation). When the angular separations from the galaxy targeted by our SPIRE/FTS observation to the companions are all (a) larger than the largest IRAS beam (i.e., $\sim 4^{\prime}$ ) or (b) smaller than or comparable to the smallest beam size of SPIRE/FTS (i.e., $\sim 17^{\prime \prime}$ ), we used the IRAS-measured total $L_{\mathrm{IR}}$ for our target. For a case in between (a) and (b), we used a reduced $L_{\mathrm{IR}}$ scaled from the IRAS total $L_{\mathrm{IR}}$ using either the Spitzer Space Observatory (hereafter Spitzer) $70 \mu \mathrm{m}$ or $24 \mu \mathrm{m}$ fluxes of the individual galaxies (Díaz-Santos et al. 2010, 2011). If the individual galaxies in the system are separated in the Spitzer $70 \mu \mathrm{m}$ image, the $70 \mu \mathrm{m}$ flux-scaled luminosities were always preferred. In this appendix, we describe how we derived $L_{\mathrm{IR}}$ in each of these cases. Note that, regardless of how $L_{\mathrm{IR}}$ was derived, the FIR color, $C(60 / 100)$, is still based on the IRAS total fluxes unless specified otherwise. Therefore, the adopted value of $C(60 / 100)$ is dominated by the brightest individual galaxy in the system. In most case, this brightest galaxy is the target in our SPIRE/ FTS observation.

NGC 0034: This is a LIRG in a galaxy pair with NGC 0035 (a non-LIRG) at an angular separation of $318^{\prime \prime}$. We used the IRAS total $L_{\mathrm{IR}}$ for our target. 


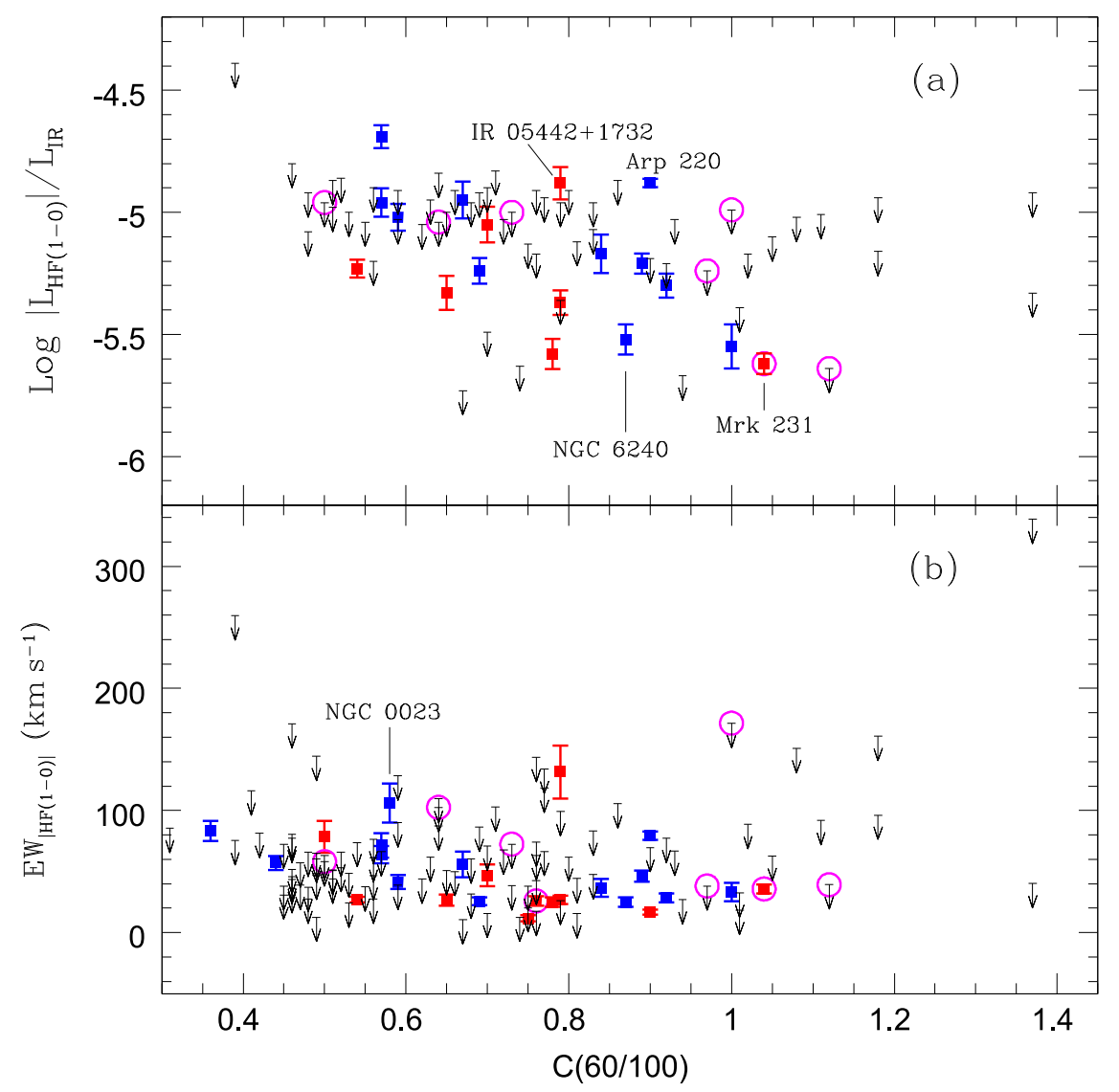

Figure 23. Plots as a function of the FIR color of (a) the ratio of the absolute $\operatorname{HF}(1-0)$ luminosity to $L_{\mathrm{IR}}$ and (b) the equivalent width (EW) of the absolute $\mathrm{HF}(1-0)$ line flux for our sample galaxies. The emission and absorption cases of detections are shown in red and blue, respectively. For non-detections, the $3 \sigma$ upper limits are shown in black. The dominant AGNs are further circled in magenta. In (a), only those targets compact enough (i.e., with $\left.f_{70 \mu \mathrm{m}}\left(17^{\prime \prime}\right)>0.8\right)$ are plotted; in (b) the whole sample is shown. Two galaxies (IRAS 05442+1732 and NGC 23), which are discussed in the text, as well as a few well-known individual galaxies, are labeled in the plots.

$M C G-02-01-051$ : This is a LIRG in a galaxy pair with MCG -02-01-052 (a non-LIRG) at an angular separation of 64". We used a $24 \mu \mathrm{m}$ flux scaled $L_{\mathrm{IR}}$ for our target, which accounts for $91 \%$ of the IRAS total $L_{\mathrm{IR}}$.

NGC 0232: This is a LIRG in a pair with NGC 0235 (a nonLIRG) at an angular separation of $121^{\prime \prime}$. The $L_{\mathrm{IR}}$ and the FIR color of this target were derived from IRAS high-resolution data in Surace et al. (2004).

NGC 0317B: This is a LIRG in a pair with NGC 0317A (a non-LIRG) at an angular separation of $33^{\prime \prime}$. We used a $24 \mu \mathrm{m}$ flux scaled $L_{\mathrm{IR}}$ for the target, which accounts for $98 \%$ of the IRAS total $L_{\mathrm{IR}}$.

IC 1623: This refers to the galaxy pair involving IC 1623A and IC $1623 \mathrm{~B}$, separated by $12^{\prime \prime}$. We used the IRAS total $L_{\mathrm{IR}}$ here.

ESO 244-G012: This refers to a galaxy pair involving two galaxies, one LIRG and one non-LIRG, separated by $17^{\prime \prime}$. We used IRAS total $L_{\mathrm{IR}}$ here.

NGC 0876: This is in a pair with NGC 0877 at an angular separation of $123^{\prime \prime}$. We used a $70 \mu \mathrm{m}$ flux scaled $L_{\mathrm{IR}}$ for our target, which accounts for $75 \%$ of the IRAS total $L_{\mathrm{IR}}$. Note that the resulting $L_{\mathrm{IR}}$ is just below the threshold for being a LIRG.

$M C G+02-08-029$ : This is in a pair with MCG +02-08-030 (a non-LIRG) at an angular separation of $22^{\prime \prime}$. We used a $24 \mu \mathrm{m}$ flux scaled $L_{\mathrm{IR}}$ for our target, which accounts for $98 \%$ of the IRAS total $L_{\mathrm{IR}}$.
UGC 02608: This is in a projected pair with a much fainter galaxy UGC 02612 with an angular separation of $247^{\prime \prime}$. We used the IRAS total $L_{\mathrm{IR}}$ for our target.

IRAS 05223+1908: The CO lines in the SPIRE/FTS spectrum of this target can be best fit with a heliocentric velocity of $100 \mathrm{~km} \mathrm{~s}^{-1}$. The PACS image at $100 \mu \mathrm{m}(\mathrm{J}$. Chu et al. 2017, in preparation) reveals three separate sources within $\sim 40^{\prime \prime}$ of the targeted position of our SPIRE/FTS observation: Object 1 (at R.A. $=5^{\mathrm{h}} 25^{\mathrm{m}} 16^{\mathrm{s}} .69$, decl. $=19^{\circ}$ $10^{\prime} 48$ "'7; J2000), Object $2\left(5^{\mathrm{h}} 25^{\mathrm{m}} 17^{\mathrm{s}} .75,19^{\circ} 10^{\prime} 12^{\prime \prime} 2\right)$, and Object $3 \quad\left(5^{\mathrm{h}} 25^{\mathrm{m}} 16^{\mathrm{s}} 40,19^{\circ} 10^{\prime} 35^{\prime \prime} \cdot 9\right)$. Our SPIRE/FTS observation was pointed at Object 1 . At $39^{\prime \prime}$ away from Object 1, Object 2 is largely outside the SPIRE/FTS beam. Object 3 is only at $13^{\prime \prime}$ from Object 1 and should contribute to the observed SPIRE/FTS spectrum. However, Object 3 is very red in color, with PACS flux densities of $0.12,0.81$ and $2.72 \mathrm{Jy}$ at 70,100 , and $160 \mu \mathrm{m}$, respectively. Our checks of the archived images from the Hubble Space Telescope, the Two-Micron All Sky Survey and Spitzer in some near- to mid-IR bands between 1.6 and $8 \mu \mathrm{m}$ in wavelength revealed no detections of this source, with quite stringent near-IR upper limits (e.g., a $3 \sigma$ limit of $27 \mathrm{mJy}$ at $3.6 \mu \mathrm{m}$ ). All these findings suggest that Object 3 is unlikely to be a Galactic source. Therefore, the CO lines detected in our SPIRE/FTS spectrum should be largely from Object 1 , which should then be a Galactic source. If Object 3 is indeed an extragalactic object, its extremely red FIR color calls for a very high 
redshift (e.g., $\gtrsim 3$ ). However, this inference seems to conflict with its relatively high PACS fluxes. Object 2 shows a quiescent FIR color based on its PACS flux densities of 1.97, 2.95, and $3.38 \mathrm{Jy}$ at 70,100 . and $160 \mu \mathrm{m}$, respectively. The IRAS $05223+1908$ system was detected in H I $21 \mathrm{~cm}$ line emission at a heliocentric velocity of $8867 \mathrm{~km} \mathrm{~s}^{-1}$ with the Arecibo radio telescope ( $\mathrm{Lu}$ et al. 1990). This H I emission might come from Object 2, as the $3 ! 2$ Arecibo beam enclosed all the three objects here.

IRAS 05442+1732: This is a pair with UGC 03356 (a nonLIRG) at an angular separation of $85^{\prime \prime}$. We used a $70 \mu \mathrm{m}$ flux scaled $L_{\mathrm{IR}}$ for our target, which accounts for $88 \%$ of the IRAS total $L_{\mathrm{IR}}$.

UGC 03410: This is in a pair with UGC 03405 (a non-LIRG) at an angular separation of $124^{\prime \prime}$. We used a $70 \mu \mathrm{m}$ flux scaled $L_{\mathrm{IR}}$, which accounts for $83 \%$ of the IRAS total $L_{\mathrm{IR}}$.

ESO 255-IG 007: This refers to two galaxies in a triple-galaxy system. In terms of the $24 \mu \mathrm{m}$ flux scaled $L_{\mathrm{IR}}$, these galaxies are (a) $6.04 \times 10^{11} L_{\odot} \quad$ (position: R.A. $=6^{\mathrm{h}} 27^{\mathrm{m}} 21.70$, decl. $=-47^{\circ} 10^{\prime} 36$ ". 2 ; J2000), (b) $1.19 \times 10^{11} L_{\odot}$ (position: $6^{\mathrm{h}} 27^{\mathrm{m}} 22^{\mathrm{s}} .55,-47^{\circ} 10^{\prime} 47^{\prime \prime} \cdot 3$ ), (c) $6.63 \times 10^{10} L_{\odot}$ (position: $\left.6^{\mathrm{h}} 27^{\mathrm{m}} 23.09,-47^{\circ} 11^{\prime} 02^{\prime \prime} 6\right)$. Our SPIRE/FTS observation was pointed at the brightest galaxy (a). The galaxies (b) and (c) are $14^{\prime \prime}$ and $30^{\prime \prime}$ off our pointing, respectively. We therefore used the summed luminosity of the two LIRGs (a) and (b) in Table 1.

NGC 2341: This is in a pair with NGC 2342 at an angular separation of $144^{\prime \prime}$. We used the IRAS high resolution data in Surace et al. (2004) to calculate the $L_{\mathrm{IR}}$ and FIR color for this target.

NGC 2342: See the note on NGC 2341 above.

$N G C$ 2388: This target has two companion galaxies: NGC 2389 at an angular offset of $202^{\prime \prime}$ and NGC 2385 at $323^{\prime \prime}$ off. We used the IRAS total $L_{\mathrm{IR}}$ for our target.

$M C G$-02-33-098: This refers to a pair system of one LIRG and one non-LIRG, separated by $11^{\prime \prime}$. The LIRG component accounts for $70 \%$ of the IRAS total $L_{\mathrm{IR}}$. we used the IRAS total $L_{\mathrm{IR}}$ here.

$N G C$ 5653: There is a companion galaxy at about $10^{\prime \prime}$ west of NGC 5653, but the companion is much fainter. We used the IRAS total $L_{\mathrm{IR}}$ here.

NGC 5734: This is in a pair with NGC 5743 (a non-LIRG) with an angular separation of $158^{\prime \prime}$. We used the IRAS highresolution data in Surace et al. (2004) to calculate the $L_{\mathrm{IR}}$ and FIR color for this target.

VV 340a: This is in a pair with VV340b at an angular separation of $39^{\prime \prime}$. Both galaxies are LIRGs. We used a $70 \mu \mathrm{m}$ flux scaled $L_{\mathrm{IR}}$ for our target, which accounts for $82 \%$ of the IRAS total $L_{\mathrm{IR}}$.

IC 4518A: This is in a pair with IC 4518B (a non-LIRG) at an angular separation of $45^{\prime \prime}$. We used a $70 \mu \mathrm{m}$ flux scaled $L_{\mathrm{IR}}$ for our target, which accounts for $84 \%$ of the IRAS total $L_{\mathrm{IR}}$.

$V V$ 705: This refers to a pair of two LIRGs with an angular separation of $7^{\prime \prime}$. We used the IRAS total $L_{\mathrm{IR}}$ here.

CGCG 052-037: This is in a pair with 2MASX J163813386827170 (a non-LIRG) at an angular separation of $68^{\prime \prime}$. We used a $70 \mu \mathrm{m}$ flux scaled $L_{\mathrm{IR}}$ for our target, which accounts for $97 \%$ of the IRAS total $L_{\mathrm{IR}}$.

NGC 6285: In a pair with NGC 6286 (a LIRG) with an angular separation of $91^{\prime \prime}$. We used the IRAS high resolution fluxes from Surace et al. (2004) to calculate the $L_{\mathrm{IR}}$ and FIR color for this target. The resulting $L_{\mathrm{IR}}$ suggests that NGC 6285 is just short of being a LIRG.

$N G C$ 6286: In a pair with NGC 6285 (a non-LIRG) with an angular separation of $91^{\prime \prime}$. We used the IRAS high resolution fluxes from Surace et al. (2004) to calculate the $L_{\mathrm{IR}}$ and FIR color for this target.

IRAS 17578-040: This has two non-LIRG companions: 2MASX J18003399-0401443 (at 60" off our SPIRE/FTS pointing) and 2MASX J18002449-040023 (at 113" off). We used a $70 \mu \mathrm{m}$ flux scaled $L_{\mathrm{IR}}$ for our target, which accounts for $83 \%$ of the IRAS total $L_{\mathrm{IR}}$ for the system.

$N G C$ 6621: This is in the Arp 81 galaxy group involving two other non-LIRG galaxies: NGC 6621SE (at 25" off our SPIRE/FTS pointing) and NGC 6622 (at 41" off). We used a $24 \mu \mathrm{m}$ flux-scaled $L_{\mathrm{IR}}$ for our target, which accounts for $97 \%$ of the IRAS total $L_{\mathrm{IR}}$.

IC 4687: This is in a galaxy system involving two other galaxies: IC 4689 (a non-LIRG at 84" off our SPIRE/FTS pointing) and IC 4686 (a LIRG at $28^{\prime \prime}$ off). We used a $24 \mu \mathrm{m}$ flux-scaled $L_{\mathrm{IR}}$ for our target, which accounts for $54 \%$ of the IRAS total $L_{\mathrm{IR}}$ for the system.

$M C G+04-48-002$ : This is in a pair with NGC 6921 (a nonLIRG) at an angular separation of $91^{\prime \prime}$. We used a $70 \mu \mathrm{m}$ flux-scaled $L_{\mathrm{IR}}$ for our target, which accounts for $78 \%$ of the IRAS total $L_{\mathrm{IR}}$.

CGCG 448-020: This refers to a triple-galaxy system consisting of two LIRGs (CGCG 448-020SEsw at R.A. $=20^{\mathrm{h}} 57^{\mathrm{m}} 24^{\mathrm{s}} .09$, and decl. $=+17^{\circ} 07^{\prime} 35^{\prime \prime} .2, \quad \mathrm{~J} 2000$; CGCG 448-020SEne at $20^{\mathrm{h}} 57^{\mathrm{m}} 24^{\mathrm{s}} .38$ and $+17^{\circ} 07^{\prime} 39^{\prime \prime}$. and a non-LIRG galaxy (CGCG 448-020NE at $20^{\mathrm{h}} 57^{\mathrm{m}} 23 \mathrm{~s} .65$ and $\left.+17^{\circ} 07^{\prime} 44^{\prime \prime} 1\right)$. All the galaxies are within $12^{\prime \prime}$ of each other. We used the IRAS total $L_{\mathrm{IR}}$ for this target.

NGC 7469: In a pair with IC 5283 (a non-LIRG) at an angular separation of $79^{\prime \prime}$. We used a $70 \mu \mathrm{m}$ flux-scaled $L_{\mathrm{IR}}$ for our target, which accounts for $86 \%$ of the IRAS total $L_{\mathrm{IR}}$. $N G C$ 7592: This refers to a galaxy pair consisting of NGC 7285E and NGC 7592W at an angular separation of $12^{\prime \prime}$. Both galaxies are LIRGs based on their $24 \mu \mathrm{m}$ fluxes. We used the IRAS total $L_{\mathrm{IR}}$.

$N G C$ 7674: This is in a pair with NGC 7674A (a non-LIRG) at an angular separation of $34^{\prime \prime}$. We used a $24 \mu \mathrm{m}$ flux-scaled $L_{\mathrm{IR}}$ for our target, which accounts for $96 \%$ of the IRAS total $L_{\mathrm{IR}}$.

NGC 7679: This is in a pair with NGC 7678 at an angular separation of $271^{\prime \prime}$. We used the IRAS total $L_{\mathrm{IR}}$ for our target. NGC 7771: This is in a triple-galaxy system with NGC 7769 and NGC 7770. Only NGC 7771 is a LIRG based on the $24 \mu \mathrm{m}$ imaging photometry. NGC 7769 is too far away to have meaningful contribution to the IRAS fluxes. The separation between NGC 7771 and NGC 7770 is $61^{\prime \prime}$. We therefore split the IRAS total $L_{\mathrm{IR}}$ between these two galaxies based on their $24 \mu \mathrm{m}$ fluxes. The resulting $L_{\mathrm{IR}}$ of NGC 7771 accounts for $76 \%$ of the IRAS total $L_{\mathrm{IR}}$.

Mrk 331: This is in a pair with UGC 12812 (a non-LIRG) at an angular separation of $118^{\prime \prime}$. We used a $24 \mu \mathrm{m}$ flux scaled $L_{\mathrm{IR}}$ for our target, which accounts for $99 \%$ of the IRAS total $L_{\mathrm{IR}}$.

\section{References}

Alatalo, K., Blitz, L., Young, L. M., et al. 2011, ApJ, 735, 88

Alonso-Herrero, A., Rieke, G. H., Rieke, M. J., \& Scoville, N. Z. 2000, ApJ, 532,845 
Alonso-Herrero, A., Roche, P. F., Esquej, P., et al. 2013, ApJL, 779, L14 Aniano, G, Draine, B. T., Gordon, K. D., \& Sandstrom, K. 2011, PASP, 123, 1218 Appleton, P. N., Guillard, P., Boulanger, F., et al. 2013, ApJ, 777, 66 Armus, L., Mazzarella, J. M., Evans, A. S., et al. 2009, PASP, 121, 559 Bayet, E., Gerin, M., Philips, T. G., \& Contursi, A. 2009, MNRAS, 399, 264 Beuther, H., Ragan, S. E., Ossenkopf, V., et al. 2014, A\&A, 571, A53 Buat, V., \& Deharveng, J. M. 1988, A\&A, 195, 60

Büttgenbach, T. H., Keene, J., Phillips, T. G., \& Walker, C. K. 1992, ApJL, 397, L15

Caputi, K. I., Lagache, G., Yan, L., et al. 2007, ApJ, 660, 97

Carilli, C. L., \& Walter, F. 2013, ARA\&A, 51, 105

da Cunha, E., Groves, B., Walter, F., et al. 2013, ApJ, 766, 13

Daddi, E., Elbaz, D., Walter, F., et al. 2010, ApJ, 714, 118

Della Ceca, R., Ballo, L., Tavecchio, F., et al. 2002, ApJL, 581, L9

Díaz-Santos, T., Armus, L., Charmandaris, V., et al. 2013, ApJ, 774, 68

Díaz-Santos, T., Armus, L., Charmandaris, V., et al. 2014, ApJ, 788, 17

Díaz-Santos, T., Armus, L., Charmandaris, V., et al. 2017, ApJ, submitted

Díaz-Santos, T., Charmandaris, V., Armus, L., et al. 2010, ApJ, 723, 993

Díaz-Santos, T., Charmandaris, V., Armus, L., et al. 2011, ApJ, 741, 32

Draine, B. T., \& Li, A. 2007, ApJ, 657, 810

Elbaz, D., Dickinson, M., Hwang, H. S., et al. 2010, A\&A, 533, A119

Feruglio, C., Fiore, F., Maiolino, R., et al. 2013a, A\&A, 549, A51

Feruglio, C., Fiore, F., Piconcelli, E., et al. 2013b, A\&A, 558, A87

Gao, Y, \& Solomon, P. M. 1999, ApJL, 512, L99

Gao, Y, \& Solomon, P. M. 2014, ApJ, 606, 271

Genzel, R., Tacconi, L. J., Gracia-Carpio, J., et al. 2010, MNRAS, 407, 2091

Gerin, M., \& Phillips, T. G. 2000, ApJ, 537, 644

Gilli, R., Norman, C., Vignali, C., et al. 2014, A\&A, 562, 67

Goicoechea, J., Martín-Pintado, J., \& Cernicharo, J. 2005, ApJ, 619, 291

González-Alfonso, E., Fischer, J., Aalto, S., \& Falstad, N. 2014a, A\&A, 567, A91

González-Alfonso, E., Fischer, J., Bruderer, S., et al. 2013, A\&A, 550, A25

González-Alfonso, E., Fischer, J., Graciá-Carpio, J., et al. 2012, A\&A, 541, A4

González-Alfonso, E., Fischer, J., Graciá-Carpio, J., et al. 2014b, A\&A, 561, A27

González-Alfonso, E., Fischer, J., Isaak, K., et al. 2010, A\&A, 518, 43

González-Alfonso, E., Smith, H. A., Ashby, M. L. N., et al. 2008, ApJ, 675,303

González-Alfonso, E., Smith, H. A., Fischer, J., \& Cernicharo, J. 2004, ApJ, 613,247

Greve, T. R., Leonidaki, I., Xilouris, E. M., et al. 2014, ApJ, 794, 142

Griffin, M. J., Abergel, A., Abreu, A., et al. 2010, A\&A, 518, L3

Gruppioni, C., Pozzi, F., Rodighiero, G., et al. 2013, MNRAS, 432, 23

Haan, S., Armus, L., Laine, S., et al. 2011, ApJS, 197, 27

Hailey-Dunsheath, S., Sturm, E., Fischer, J., et al. 2012, ApJ, 755, 57

Harrison, A., Puxley, P., Russell, A., \& Brand, P. 1995, MNRAS, 277, 413

Heckman, T. M., Armus, L., \& Miley, G. K. 1987, AJ, 93, 276

Helou, G. 1986, ApJL, 311, L33

Henkel, C., Peck, A. B., Tarchi, A., et al. 2005, A\&A, 436, 75

Howell, J. H., Armus, L., Mazzarella, J. M., et al. 2010, ApJ, 715, 572

Ikeda, M., Oka, T., Tatematsu, K., Sekimoto, Y., \& Yamamoto, S. 2002, ApJS, 139,467

Inami, H., Armus, L., Charmandaris, V., et al. 2013, ApJ, 777, 156

Israel, F. P., \& Baas, F. 2002, A\&A, 383, 82

Iwasawa, K., Sanders, D. B., Teng, S. H., et al. 2011, A\&A, 529, A106

Kamenetzky, J., Glenn, J., Rangwala, N., et al. 2012, ApJ, 753, 70

Kamenetzky, J., Rangwala, N., Glenn, J., Maloney, P. R., \& Conley, A. 2016, ApJ, 829, 93

Kaufman, M. J., Wolfire, M. G., Hollenbach, D. J., \& Luhman, M. L. 1999, ApJ, 527, 795

Lagos, C. P., Bayet, E., Baugh, C. M., et al. 2012, MNRAS, 426, 2142

Lanz, L., Ogle, P. M., Evans, D., et al. 2015, ApJ, 801, 17

Laurent, O, Mirabel, I. F., Charmandaris, V., et al. 2000, A\&A, 359, 887

Le Flóch, E., Papovich, C., Dole, H., et al. 2005, ApJ, 632, 169

Leech, J., Isaak, K. G., Papadopoulos, P. P., Gao, Y., \& Davis, G. R. 2010, MNRAS, 406, 1364

Liu, D., Gao, Y., Isaak, K., et al. 2015, ApJL, 810, L14

Lu, N., Dow, M. W., Houck, J. R., Salpeter, E. E., \& Lewis, B. M. 1990, ApJ, 357,388

Lu, N., Zhao, Y., Xu, C. K., et al. 2014, ApJL, 787, L23

Lu, N., Zhao, Y., Xu, C. K., et al. 2015, ApJL, 802, L11

Lutz, D., Berta, S., Contursi, A., et al. 2016, A\&A, 591, A136

Magnelli, B., Elbaz, D., Chary, R. R., et al. 2009, A\&A, 496, 57

Magnelli, B., Elbaz, D., Chary, R. R., et al. 2011, A\&A, 528, A35

Markwardt, C. B. 2009, adass XVIII, 411, 251

Mashian, N., Sturm, E., Sternberg, A., et al. 2015, ApJ, 802, 81

Max, C. E., Canalizo, G., Macintosh, B. A., et al. 2005, ApJ, 621, 738
Meijerink, R., Kristensen, L. E., Weiß, A., et al. 2013, ApJL, 762, L16

Monje, R. R., Emprechtinger, M., Phillips, T. G., et al. 2011, ApJ, 734, 23

Monje, R. R., Lord, S., Falgarone, E., et al. 2014, ApJ, 785, 22

Murphy, E. J., Stierwalt, S., Armus, L., Condon, J. J., \& Evans, A. S. 2013, ApJ, 768, 2

Muzzin, A., van Dokkum, P., Kriek, M., et al. 2010, ApJ, 725, 742

Neufeld, D. A., Sonnentrucker, P., Phillips, T. G., et al. 2010, A\&A, 518, L108

Neufeld, D. A., Wolfire, M., \& Schilke, P. 2005, ApJ, 628, 260

Ogle, P., Boulanger, F., Guillard, P., et al. 2010, ApJ, 1193

Ojha, R., Stark, A. A., Hsieh, H. H., et al. 2001, ApJ, 548, 253

Ott, S. 2010, in ASP Conf. Ser. 434, Astronomical Data Analysis Software and

Systems XIX, ed. Y. Mizumoto, K. Morita, \& M. Ohishi (San Francisco,

CA: Astronomical Society of the Pacific), 139

Panuzzo, P., Rangwala, N., Rykala, A., et al. 2010, A\&A, 518, L37

Papadopoulos, P. P., \& Greve, T. R. 2004, ApJL, 615, L29

Papadopoulos, P. P., Thi, W.-F., \& Viti, S. 2004, MNRAS, 351, 147

Papadopoulos, P. P., van der Werf, P., Xilouris, E., Isaak, K. G., \& Gao, Y. 2012, ApJ, 751, 10

Papadopoulos, P. P., Zhang, Z.-Y., Xilouris, E. M., et al. 2014, ApJ, 788, 153 Pellegrini, E. W., Smith, J. D., Wolfire, M. G., et al. 2013, ApJL, 779, L19

Pereira-Santaella, M., Spinoglio, L., Busquet, G., et al. 2013, ApJ, 768, 55

Petric, A. O., Armus, L., Howell, J., et al. 2011, ApJ, 730, 28

Petty, S. M., Armus, L., Charmandaris, V., et al. 2014, AJ, 148, 111

Phillips, T. G., Bergin, E. A., Liz, D. C., et al. 2010, A\&A, 518, 109

Pilbratt, G. L., Riedinger, J. R., Passvogel, T., et al. 2010, A\&A, 518, L1

Poglitsch, A., Waelkens, C., Geis, N., et al. 2010, A\&A, 518, L2

Privon, G. C., Herrero-Illana, R., Evans, A. S., et al. 2015, ApJ, 814, 39

Rangwala, N., Maloney, P. R., Glenn, J., et al. 2011, ApJ, 743, 94

Rawle, T. D., Egami, E., Bussmann, R. S., et al. 2014, ApJ, 783, 59

Riechers, D. A., Bradford, C. M., Clements, D. L., et al. 2013, Natur, 496, 329

Rigopoulou, D., Hurley, P. D., Swinyard, B. M., et al. 2013, MNRAS, 434, 2051

Rosenberg, M. J. F., Kazandjian, M. V., van der Werf, P. P., et al. 2014a, A\&A, 564, A126

Rosenberg, M. J. F., Meijerink, R., Israel, F. P., et al. 2014b, A\&A, 568, A90

Rosenberg, M. J. F., van der Werf, P. P., Aalto, S., et al. 2015, A\&A, 568, A90

Rujopakarn, W., Rieke, G. H., Eisenstein, D. J., \& Juneau, S. 2011, ApJ, 726, 93

Sakamoto, K., Aalto, S., Costagliola, F., et al. 2013, ApJ, 764, 42

Sanders, D. B., Mazzarella, J. M., Kim, D.-C., \& Surace, J. A. 2003, ApJ, 126,1607

Sanders, D. B., \& Mirabel, I. F. 1996, ARA\&A, 34, 749

Sanders, D. B., Scoville, N. Z., \& Soifer, B. T. 1991, ApJ, 370, 158

Sargent, A. L., \& Scoville, N. 1991, ApJL, 366, L1

Schilke, P., Carlstrom, J. E., Keene, J., \& Phillips, T. 1993, ApJL, 417, L67

Schirm, M. R. P., Wilson, C. D., Parkin, T. J., et al. 2014, ApJ, 781, 101

Scoville, N., Sheth, K., Walter, F., et al. 2015, ApJ, 800, 70

Sonnentrucker, P, Neufeld, D. A., Phillips, T. G., et al. 2010, A\&A, 521, L12

Sonnentrucker, P, Wolfire, M., Neufeld, D. A., et al. 2015, ApJ, 806, 49

Spaans, M., \& Meijerink, R. 2008, ApJL, 678, L5

Spinoglio, L., Pereira-Santaella, M., Busquet, G., et al. 2012, ApJ, 758, 108

Stierwalt, S., Armus, L., Charmandaris, V., et al. 2014, ApJ, 790, 124

Stierwalt, S., Armus, L., Surace, J. A., et al. 2013, ApJS, 206, 1

Stutzki, J, Graf, U. U., Haas, S., et al. 1997, ApJL, 477, L33

Surace, J. A., Sanders, D. B., \& Mazzarella, J. M. 2004, ApJ, 127, 3235

Swinyard, B. M., Polehampton, E. T., Hopwood, R., et al. 2014, MNRAS, 440, 3658

Tacconi, L. J., Genzel, R., Tecza, M., et al. 1999, ApJ, 524, 732

Tarchi, A., Castangia, P., Henkel, C., \& Menten, K. M. 2007, NewAR, 51, 67 Telesco, C. M., \& Decher, R. 1988, ApJ, 334, 573

U, V., Sanders, D. B., Mazzarella, J. M., et al. 2012, ApJS, 203, 9

Van der Tak, F. F. S., Ossenkopf, V., Nagy, Z., et al. 2012, A\&A, 537, L10

van der Werf, P. P., Isaak, K. G., Meijerink, R., et al. 2010, A\&A, 518, L42 van Dishoeck, E. F., Kristensen, L. E., Benz, A. O., et al. 2011, PASP, 123, 138

Veilleux, S., Pupke, D. S. N., Kim, D.-C., et al. 2009, ApJS, 182, 628

Weiß, A., Henkel, C., Downes, D., \& Walter, F. 2003, A\&A, 409, L41

Weiß, A., Requena-Torres, M. A., Güsten, R., et al. 2010, A\&A, 521, L1

Wu, J., Evans, N. J., II, Gao, Y., et al. 2005, ApJ, 635, 173

Wu, R., Madden, S. C., Galliano, F., et al. 2015, A\&A, 575, A88

Wu, R., Polehampton, E. T., Etxaluze, M., et al. 2013, A\&A, 556, A116

Xu, C. K, Cao, C., Lu, N., et al. 2014, ApJ, 787, 48

Xu, C. K., Cao, C., Lu, N., et al. 2015, ApJ, 799, 11

Yang, C., Gao, Y., Omont, A., et al. 2013, ApJL, 771, L24

Yao, L., Seaquist, E. R., Kuno, N., \& Dunne, L. 2003, ApJ, 588, 771

Zhao, Y., Lu, N., Xu, C. K., et al. 2013, ApJL, 765, L13

Zhao, Y., Lu, N., Xu, C. K., et al. 2016a, ApJ, 819, 69

Zhao, Y., Lu, N., Xu, C. K., et al. 2016b, ApJ, 820, 118 